HUTP-01/A041

ITEP-TH-47/01

DAMTP-2001-77

hep-th/0109025

\title{
M-Theory on Spin(7) Manifolds
}

\author{
Sergei Gukov* \\ Jefferson Physical Laboratory, Harvard University \\ Cambridge, MA 02138, USA \\ JAMES SPARKS ${ }^{\dagger}$ \\ Centre for Mathematical Sciences \\ University of Cambridge \\ Wilberforce Road, Cambridge CB3 OWA, UK.
}

February 1, 2008

\begin{abstract}
We study M-theory on two classes of manifolds of Spin(7) holonomy that are developing an isolated conical singularity. We construct explicitly a new class of Spin(7) manifolds and analyse in detail the topology of the corresponding classical spacetimes. We discover also an intricate interplay between various anomalies in M-theory, string theory, and gauge theory within these models, and in particular find a connection between half-integral $G$-fluxes in M-theory and Chern-Simons terms of the $\mathcal{N}=1, D=3$ effective theory.
\end{abstract}

*email: gukov@democritus.harvard.edu

†email: J.F.Sparks@damtp.cam.ac.uk 


\section{Contents}

\begin{tabular}{lll}
\hline 1 & Introduction and Summary & 3
\end{tabular}

\begin{tabular}{|lll}
\hline 2 & Riemannian Manifolds of Special Holonomy & 7
\end{tabular}

2.1 The Holonomy Groups G2 and Spin(7) . . . . . . . . . . . . . . . . . . 7

2.2 G2 Manifolds . . . . . . . . . . . . . . . . . . . . . . . . . . . 8

$2.3 \quad$ Spin(7) Manifolds . . . . . . . . . . . . . . . . . . . . . . . . . . . . . . 9

3 Topological Charges And Relation To Singularities Of Calibrated Cycles 11

3.1 Circle Quotients . . . . . . . . . . . . . . . . . . . . . . . 11

3.2 Identification of Topological Charges . . . . . . . . . . . . . . . . . . . 14

4 New Complete Non-Compact Spin(7) Manifolds 24

4.1 Aloff-Wallach Spaces . . . . . . . . . . . . . . . . . . . . . . . 25

4.2 The Relation to Spinc Structures . . . . . . . . . . . . . . . . . . . . . 29

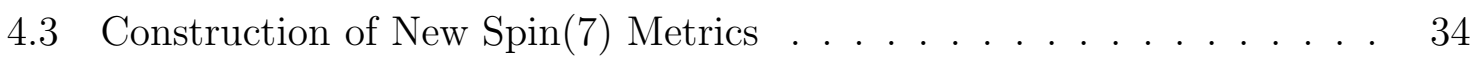

4.4 New $\operatorname{Spin}(7)$ Metrics on Q . . . . . . . . . . . . . . . . . . . . 37

5 Flux Quantisation in M-Theory on Spin(7) Manifolds 39

5.1 D6-branes Wrapping Coassociative Cycles . . . . . . . . . . . . . . . 40

5.2 Flux Quantisation . . . . . . . . . . . . . . . . . . . . . . . . 42

5.3 G-Flux from K-Theory . . . . . . . . . . . . . . . . . . . . . . . . . . . 44

$6 \quad$ Effective $\mathcal{N}=1$ Three-Dimensional Field Theory 48

6.1 Compactification on a General Spin(7) Manifold . . . . . . . . . . . . . 49

6.2 Chern-Simons Terms Induced by G-Flux . . . . . . . . . . . . . . . . . 54

6.3 M-Theory on $\mathbb{B}_{8} \ldots \ldots \ldots \ldots$

$6.4 \quad$ M-Theory on $\operatorname{Spin}^{c}$ Bundles over $\mathbb{C P}^{2}$. . . . . . . . . . . . . . . . . . 67

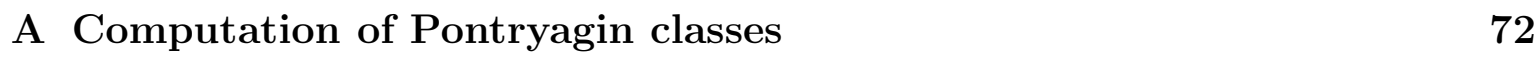

\begin{tabular}{|ll}
\hline B Joyce Construction of Spin(7) Manifolds & 74
\end{tabular} 


\section{Introduction and Summary}

Recently, M-theory compactifications on manifolds of exceptional holonomy have attracted considerable attention. These models allow one to geometrically engineer various minimally supersymmetric gauge theories, which typically have a rich dynamical structure. A particularly interesting aspect of such models is the behaviour near a classical singularity, where one might expect extra massless degrees of freedom, enhancement of gauge symmetry, or a phase transition to a different theory.

In the case of $G 2$ compactifications one obtains an $\mathcal{N}=1$ supersymmetric field theory in four dimensions, where certain properties of the IR quantum theory can be obtained from non-renormalization theorems, holomorphy, and R-symmetry. Using holomorphy constraints, Atiyah, Maldacena, and Vafa [1] argued that in the quantum theory one can smoothly interpolate between certain spacetime manifolds of $G 2$ holonomy which have three classical limits. Each of these classical limits can be understood as an M-theory lift of Type IIA string theory on a deformed/resolved conifold with D6branes/RR-flux in the background. Therefore, the smooth geometric transition found in M-theory implies that in Type IIA one has a continuous transition from a vacuum with D6-branes to another vacuum where the branes have disappeared and have been replaced with RR-flux.

More evidence in favour of a smooth transition in this model was presented in the recent work of Atiyah and Witten [2], where M-theory dynamics on other known G2 holonomy manifolds was also discussed. Specifically, these are resolutions of the cones on $\mathbb{C P}^{3}$ and on $S U(3) / U(1)^{2}$. In both cases one has a collapsing four-cycle $\left(\mathbf{S}^{4}\right.$ and $\mathbb{C P}^{2}$, respectively) in the limit that the $G 2$ manifold develops a conical singularity. Unlike the model considered in [1], these manifolds do not have an interpretation as M-theory lifts of D6-branes wrapped on non-compact, topologically non-trivial CalabiYau manifolds. However, the dynamics of these models can be obtained from a different reduction to Type IIA theory with D6-branes in a (topologically) flat spacetime [2]. Via this reduction, the problem of studying M-theory on a G2 manifold developing a conical singularity can be translated into the simpler problem of studying configurations of intersecting D6-branes in flat spacetime. In particular, for the cone on $\mathbb{C P}^{3}$ one finds restoration of a global $U(1)$ symmetry at the conifold point, whereas for the $S U(3) / U(1)^{2}$ model there are three different branches related by a "triality" symmetry. In fact, there is a close relation between the $\operatorname{Spin}(7)$ examples in the current paper and the $G 2$ models of [2].

In the case of $\operatorname{Spin}(7)$ compactifications there are fewer constraints from supersymmetry. Namely, compactification of M-theory on a $\operatorname{Spin}(7)$ manifold gives $\mathcal{N}=1$ supersymmetric field theory in three dimensions. This theory cannot be obtained via 
dimensional reduction from four dimensions. One might hope to use this fact to explain the vanishing of the cosmological constant in four dimensions in the absence of supersymmetry, along the lines of [3, 4 . Scalar fields in $\mathcal{N}=1$ multiplets are real in three dimensions. So, there is no holomorphy, and in general one would not expect smooth transitions between different branches similar to the phase transitions in the $G 2$ case [1, 5, 2]. Moreover, there are no non-renormalization theorems and no R-symmetries in $\mathcal{N}=1$ three-dimensional theories. However, certain constraints may be obtained from the discrete parity symmetry [6, 7, 8]:

$$
P:\left(x^{0}, x^{1}, x^{2}\right) \rightarrow\left(x^{0},-x^{1}, x^{2}\right)
$$

For example, in a parity-invariant theory the superpotential is odd under this transformation [6]. Important questions, such as dynamical supersymmetry breaking in $\mathcal{N}=1$ three-dimensional theories, may also be addressed by studying the supersymmetric index [9] and supergravity duals of these theories [10].

Motivated by [1, 2], in this paper we study M-theory dynamics on manifolds of Spin(7) holonomy which are developing an isolated conical singularity. Until recently, only one example of this type was known, corresponding to a cone on $\mathbf{S}^{7}=S O(5) / S O(3)$ [11]. Existence of many other complete metrics of $\operatorname{Spin}(7)$ holonomy can be conjectured, as in the $G 2$ case [1], by lifting D6-brane configurations to M-theory [12]. Specifically, one starts with Type IIA string theory on $M^{3} \times M^{7}$, where $M^{3}$ is a $(2+1)$-dimensional spacetime $\rrbracket$, and $M^{7}$ is a (non-compact) 7-manifold with $G 2$ holonomy. This gives $\mathcal{N}=2$ supersymmetric field theory (without gravity) on $M^{3}$. Let us further assume that $M^{7}$ has a topologically non-trivial supersymmetric 4-cycle $B$, known as a coassociative cycle [13, 14], and let us introduce a space-filling D6-brane with world-volume $M^{3} \times B$. Since $B$ is supersymmetric, we obtain an $\mathcal{N}=1$ effective field theory in three dimensions. Now consider the M-theory lift of this system. The eleven-dimensional metric should look like $M^{3} \times X$, where $X$ is a (degenerate) circle bundle over $M^{7}$. Moreover, since a D6-brane lifts to a purely geometric background (Taub-NUT space), one can roughly speaking think of $X$ as a Taub-NUT bundle over $B$, such that $X$ preserves only two real supercharges, i.e. $\operatorname{Hol}(X)=\operatorname{Spin}(7)$. A metric with $\operatorname{Spin}(7)$ holonomy obtained in this way should be asymptotically locally conical (ALC), since the size of the $\mathbf{S}^{1}$ fiber at large distance is related to the Type IIA coupling constant and, therefore, should be finite. Furthermore, the circle should degenerate on a codimension four submanifold $B$, describing the D6-brane locus.

A complete asymptotically locally conical metric with these properties was constructed in the case $B=\mathbf{S}^{4}$ in [15]. This solution describes D6-branes wrapped on a coassociative 4-sphere in the total space of $\Lambda^{-} \mathbf{S}^{4}$, the bundle of anti-self-dual two-forms

\footnotetext{
${ }^{1} M^{3}$ is usually assumed to be either $\mathbb{R}^{3}$ or $\mathbb{R}^{1} \times T^{2}$, unless otherwise stated.
} 
over $\mathbf{S}^{4}$. In this paper we explicitly construct another family of new ALC metrics with $B=\mathbb{C P}^{2}$. After reduction to Type IIA these metrics represent D6-branes wrapped on the supersymmetric $\mathbb{C} \mathbf{P}^{2}$ inside $\Lambda^{-} \mathbb{C} \mathbf{P}^{2}$. These are the two main examples of noncompact $\operatorname{Spin}(7)$ manifolds that we analyze in this paper. Note that in both examples we have D6-branes on one of the asymptotically conical $G 2$ holonomy manifolds studied in $[2]$.

In both cases $\left(B=\mathbf{S}^{4}\right.$ and $\left.B=\mathbb{C P}^{2}\right)$ the non-compact $\operatorname{Spin}(7)$ manifold is homotopy equivalent to $\mathbb{R}^{4} \times B$ with level surfaces (constant $r$ surfaces) $Y=S O(5) / S O(3)$ and $Y=S U(3) / U(1)$, respectively. In the limit when the 4-cycle $B$ shrinks to zero size, the 8-manifold $X$ develops an isolated conical singularity. Since in this limit the physics is described by the local behaviour near the singularity, we usually take $X$ to be a cone over the appropriate weak $G 2$ manifold $Y$. However, it is useful to bear in mind that M-theory on $X$ can be thought of as a configuration of D6-branes wrapped on the coassociative 4-cycle in the corresponding topologically non-trivial $G 2$ manifold $M^{7}$.

There is another reduction to Type IIA theory that will be very useful in our discussion. As for the $G 2$ case [2], one can find a semi-free $U(1)$ action on $X$ such that $X / U(1)$ is topologically trivial, i.e. $X / U(1) \cong \mathbb{R}^{7}$. Following [2], we denote the fixed point set of such a $U(1)$ action as $L$. The space $L$ has real dimension four and represents the location of space-filling D6-branes.

To summarise, one may think of M-theory on the non-compact Spin(7) manifolds discussed here in several equivalent ways:

- M-theory on a manifold $X$ of $\operatorname{Spin}(7)$ holonomy;

- Type IIA theory on a $G 2$-holonomy manifold $M^{7}=\Lambda^{-} B$ with D6-branes wrapped over the supersymmetric 4-cycle $B$;

- A supersymmetric configuration of D6-branes in Type IIA theory with worldvolume:

$$
M^{3} \times L \subset M^{3} \times \mathbb{R}^{7}
$$

The paper is organised as follows. In order to make the paper self-contained, we begin in section 2 with a brief review of special holonomy manifolds and describe in outline the existing examples of explicitly known metrics relevant to our discussion.

In section 3 we describe the reduction of our models to configurations of D6-branes in flat spacetime. We study the spectrum of topologically stable objects, such as solitons, domain walls, etc., in Type IIA and in M-theory. Identifying the corresponding states in the spectrum, we find a simple relation between the homology groups of the D6brane locus $L$ and those of the $\operatorname{Spin}(7)$ manifold $X$. Global world-sheet anomalies and knotted 3-spheres inside $\mathbf{S}^{7}$ enter our discussion in a natural way. 


\begin{tabular}{|c|c|c|}
\hline Spin(7) Manifold $X$ & $\Sigma_{-} \mathbf{S}^{4} \cong \mathbb{R}^{4} \times \mathbf{S}^{4}$ & $\mathcal{Q} \cong \mathbb{R}^{4} \times \mathbb{C P}^{2}$ \\
\hline \hline Principal Orbit $Y$ & $S O(5) / S O(3)$ & $S U(3) / U(1)$ \\
\hline Collapsing Cycle $B$ & $\mathbf{S}^{4}$ & $\mathbb{C} \mathbf{P}^{2}$ \\
\hline$U(1)$ Bundle over $G 2$ Manifold $M^{7}$ & $\Lambda^{-} \mathbf{S}^{4}$ & $\Lambda^{-} \mathbb{C P}^{2}$ \\
\hline D6-Brane Locus $L$ & $\mathbb{R}^{2} \times \mathbf{S}^{2}$ & $\mathbb{R}^{4} \cup \mathbb{R}^{2} \times \mathbf{S}^{2}$ \\
\hline Global Symmetry & $S p(2) \times_{\mathbb{Z}_{2}} S p(1) \times \mathbb{Z}_{2}$ & $S U(3) \times U(1) \times U(1)$ \\
\hline Is Modulus Dynamical? $_{\text {Bo }}$ & No \\
\hline Background Flux, $\int_{B} G^{(4)}$ & $\mathbb{Z}+1 / 2$ & $\mathbb{Z}+1 / 2$ \\
\hline Number of Massive Vacua & 1 & 1 or 2 \\
\hline
\end{tabular}

Table 1: The two examples of non-compact manifolds of $\operatorname{Spin}(7)$ holonomy studied in this paper.

In section 4 we explicitly construct a new family of complete $\operatorname{Spin}(7)$ metrics on a certain $\mathbb{R}^{4}$ bundle over $\mathbb{C P}^{2}$, known as the universal quotient bundle $\mathcal{Q}$. This solution has recently been extended [16]. We study in detail the global topology of these solutions, whose level surfaces are various so-called Aloff-Wallach spaces $N_{k, l}=S U(3) / U(1)$, where the integers $k$ and $l$ (such that $k l \neq 0$ ) parametrise the embedding of $U(1)$ in $S U(3)$. Every pair $(k, l)$ corresponds to a distinct Spin(7) manifold, which, modulo discrete identifications, is the total space of a $\operatorname{spin}^{c}$ structure on $\mathbb{C} \mathbf{P}^{2}$. We also discuss the action of the "triality" group $\Sigma_{3}$ of permutations of three elements on these spaces. A systematic approach to the construction of new exceptional holonomy metrics can be found in [17].

In sections 5 and 6 we discuss various M-theoretic aspects of our work. In section 5 we describe how the M-theory lift of a configuration of D6-branes wrapping a coassociative cycle is related to $\operatorname{spin}^{c}$ bundles, and also discuss $G$-flux quantisation [18]. In particular, we find that the $G$-flux obeys a shifted quantisation condition, and has to be half-integer in our models. This shift is related to the K-theory classification of RR-fields in Type IIA string theory. In section 6 we explain the relation between the anomalous shift in the $G$-flux quantisation condition and the shift of the Chern-Simons coefficient in the effective $\mathcal{N}=1$ gauge theory in $(2+1)$ dimensions. We also study certain dynamical aspects of M-theory on (singular) Spin(7) manifolds, which we summarise in table 1.

Note Added: Recently we received a preprint [19] that significantly overlaps with sections 4.3 and 4.4 of the present paper, which were completed some time ago. This has prompted us to publish the paper in two parts, of which this is the first. A second paper will contain a more detailed analysis of various aspects of M-theory on $\operatorname{Spin}(7)$ manifolds [20]. An extension of some of the results in section 4 of this paper may also 
be found in the recent publication [16].

\section{Riemannian Manifolds of Special Holonomy}

In this section we review the metrics of $G 2$ and $\operatorname{Spin}(7)$ holonomy constructed in [11, together with the recent examples of $\operatorname{Spin}(7)$ manifolds constructed in [15].

\subsection{The Holonomy Groups G2 and Spin(7)}

The holonomy group $H$ of a generic oriented Riemannian $n$-manifold $Y$ is the special orthogonal group, $S O(n)$. However, if $H$ is a proper subgroup of $S O(n)$ then the manifold $Y$ will inherit special geometric properties. These properties are typically characterised by the existence of non-degenerate (in some suitable sense) $p$-forms which are covariantly constant. Such $p$-forms also serve as calibrations, and are related to the subject of minimal varieties.

The possible choices for $H \subset S O(n)$ are limited. Specifically, Berger's Theorem tells us that, for $Y$ simply-connected and neither locally a product nor symmetric, the only possibilities for $H$, other than the generic case of $S O(n)$, are $U\left(\frac{n}{2}\right), S U\left(\frac{n}{2}\right)$, $S p\left(\frac{n}{4}\right) S p(1), S p\left(\frac{n}{4}\right), G 2$ 2, $\operatorname{Spin}(7)$ or $\operatorname{Spin}(9)$. The first four of these correspond, respectively, to a Kähler, Calabi-Yau, Quaternionic Kähler or hyper-Kähler manifold. The last three possibilities are the exceptional cases, occuring only in dimensions 7,8 and 16, respectively. The latter case is in some sense trivial in that any 16-manifold of $\operatorname{Spin}(9)$ holonomy is locally isometric to the Cayley projective plane, $\mathbb{O} \mathbf{P}^{2}$ (or its dual).

In the present paper, we shall be interested in both $G 2$ and $\operatorname{Spin}(7)$ manifolds; that is, Riemannian manifolds with holonomy group $G 2$ and $\operatorname{Spin}(7)$, respectively. The local existence of such manifolds was first demonstrated by Bryant, although a more thorough treatment, which we review briefly in the next two subsections, was given in [11]. The first examples of metrics with $G 2$ and $\operatorname{Spin}(7)$ holonomy on compact manifolds were constructed by Joyce [21]. We note in passing that $G 2$ and $\operatorname{Spin}(7)$ manifolds are always Ricci flat.

On a $G 2$ manifold $M^{7}$, there exists a distinguished harmonic three-form $\Psi$, the associative three-form, which locally determines the reduction of the structure group $\operatorname{Spin}(7)$ to $G 2$. The Hodge dual form $* \Psi$, is therefore also harmonic, and is referred to as the coassociative four-form. Similarly, on a Spin(7) manifold $X$, there exists

\footnotetext{
${ }^{2}$ The fourteen-dimensional simple Lie group $G 2 \subset \operatorname{Spin}(7)$ is precisely the automorphism group of the octonions, $\mathbb{O}$.
} 
a distinguished self-dual harmonic four-form, the Cayley form, $\Phi=* \Phi$, that locally determines the reduction of the structure group $\operatorname{Spin}(8)$ to $\operatorname{Spin}(7)$.

The G2 and $\operatorname{Spin}(7)$ conditions may also be characterised by examining the behaviour of spinors under the decomposition of the structure group. Specifically, for a G2 manifold, the decomposition of the Majorana 8 of $\operatorname{Spin}(7)$ under $G 2$ is

$$
8 \rightarrow 7+1
$$

The singlet 1 corresponds to a parallel spinor; that is, a covariantly constant section

of the appropriate spin bundle. Similarly, for a Spin(7) manifold, the decomposition

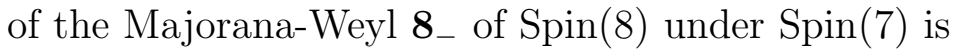

$$
8_{-} \rightarrow 7+1
$$

The singlet is again a parallel spinor. Note that the $\mathbf{8}_{+}$then decomposes irreducibly.

We conclude this subsection by reminding the reader of the definition of a calibration. A closed $p$-form $\phi$ is said to be a calibration if the restriction of $\phi$ to each tangent $p$-plane is less than or equal to the volume form of that $p$-plane. A $p$-dimensional submanifold on which equality is obtained, at each point, is then referred to as a calibrated submanifold (with respect to the calibration $\phi$ ). It is then a trivial exercise to show that a calibrated submanifold is volume-minimising within its homology class, and is therefore stable. The forms $\Psi$ and $* \Psi$ both serve as calibrations on a $G 2$ manifold, the calibrated submanifolds being respectively referred to as associative or coassociative submanifolds. Likewise, the Cayley form $\Phi$ is a calibration for a $\operatorname{Spin}(7)$ manifold, the calibrated submanifolds then being referred to as Cayley submanifolds. The deformability of calibrated submanifolds was studied by McLean [22] and will be relevant in the present paper. The calibrations themselves may be constructed using the parallel spinors, essentially by "squaring" them.

\section{$2.2 \quad$ G2 Manifolds}

In this section, we briefly summarise the properties of the two known complete noncompact $G 2$ manifolds that contain a coassociative submanifold, [11].

Let us start by considering the consequences of the existence of a coassociative submanifold, $B$. We begin with some preliminaries. If $B$ is a closed oriented Riemannian four-manifold, we denote the bundle of $p$-forms over $B$ as $\Lambda^{p} \equiv \Lambda^{p} T^{*} B$. The Hodge map on $B$ induces a direct sum decomposition $\Lambda^{2}=\Lambda^{+} \oplus \Lambda^{-}$, where the rank three vector bundles $\Lambda^{ \pm}$are the bundles of self-dual and anti-self-dual two-forms on $B$. Note 
that since the Hodge map acting on middle-dimensional forms is invariant under conformal rescalings of the metric, the decomposition only depends on the conformal class of the metric on $B$. In [22], it was shown that the normal bundle $N B$ of a coassociative submanifold $B$ in $M^{7}$ is isomorphic to the bundle of anti-self-dual two-forms over $B$, $N B \cong \Lambda^{-} B$.

In [11], complete Ricci-flat metrics of $G 2$ holonomy were constructed on the total spaces of the bundles of anti-self-dual two-forms over $\mathbf{S}^{4}$ and $\mathbb{C} \mathbf{P}^{2}$, the zero-section, or bolt, being a coassociative submanifold in each case. The authors chose a cohomogeneity one ansatz for the metric, so that the Ricci-flatness condition reduces to a coupled system of second order differential equations for the metric functions, in terms of the the radial variable $r$. In light of the above comments, the level surfaces $\{r=$ constant $\}$ must be topologically the bundle of unit vectors $\mathcal{S} \Lambda^{-} B$, in $\Lambda^{-} B$. This is known as the twistor space of $B$ [23] and is an $\mathbf{S}^{2}$ bundle over $B$.

The one-paramater family of metrics are given explicitly by

$$
d s^{2}=\left(1-\left(\frac{a}{r}\right)^{4}\right)^{-1} d r^{2}+\frac{1}{4} r^{2}\left(1-\left(\frac{a}{r}\right)^{4}\right)\left(D \mu^{i}\right)^{2}+\frac{1}{2} r^{2} d \Omega_{4}^{2}
$$

where $\mu^{i}$ are coordinates on $\mathbb{R}^{3}$, subject to the constraint $\mu^{i} \mu^{i}=1$ (thus yielding the $\mathbf{S}^{2}$ fibre), and the covariant derivative is $D \mu^{i}=d \mu^{i}+\epsilon_{i j k} A^{j} \mu^{k}$ where $A^{i}$ is the $S U(2)$ connection on the four-dimensional (Quaternionic Kähler) Einstein manifold with metric $d \Omega_{4}^{2}$; that is, the field strengths $J^{i}=d A^{i}+\frac{1}{2} \epsilon_{i j k} A^{j} \wedge A^{k}$ satisfy the unit quaternion algebra. We may take $d \Omega_{4}^{2}$ to be either $\mathbf{S}^{4}$ or $\mathbb{C} \mathbf{P}^{2}$, so that the conformal class of the metric on $B$ is the canonical one in each case. The $G 2$ metric is complete on the region $r \geq a$, with $r=a$ the coassociative submanifold, and the principal orbits $\{r=$ constant $\}$ are respectively $\mathbb{C P}^{3}$ and $S U(3) / T^{2}$ where $T^{2}$ is a maximal torus in $S U(3)$; these are precisely the twistor spaces of $\mathbf{S}^{4}$ and $\mathbb{C} \mathbf{P}^{2}$. Indeed, the metric is asymptotic to the cone over the squashed (nearly Kähler, rather than the Kähler Fubini-Study) Einstein metric on $\mathbb{C P}^{3}$, or the squashed (nearly Kähler) metric on $S U(3) / T^{2}$, respectively.

\section{$2.3 \quad \operatorname{Spin}(7)$ Manifolds}

Until recently only one complete non-compact Spin(7) manifold was explicitly known, and was originally constructed along with the above G2 manifolds in [11]. This construction has recently been extended 15 to yield a new family of Spin(7) manifolds. We also discuss the Spin(7) orbifold discovered in [24]. The construction of this particular solution is in fact a special case of the construction used in the present paper to find a new family of $\operatorname{Spin}(7)$ metrics on a certain $\mathbb{R}^{4}$ bundle over $\mathbb{C P}^{2}$. 
The Spin(7) manifold presented in 11] contains a Cayley submanifold, which is an $\mathbf{S}^{4}$. In [22], it was shown that the normal bundle of an $\mathbf{S}^{4}$ Cayley submanifold in a $\operatorname{Spin}(7)$ manifold $X$ is topologically $N \mathbf{S}^{4}=\Sigma_{-} \mathbf{S}^{4}$, the bundle of negative chirality spinors over $\mathbf{S}^{4}$. The $\operatorname{Spin}(7)$ manifold presented in [11 is in fact the total space of this normal bundle, as was the case for the $G 2$ metrics in the last subsection. The metric is again cohomogeneity one, with level surfaces $\{r=$ constant $\}$ being topologically $\mathbf{S}^{7}$, described as an $\mathbf{S}^{3}=S U(2)$ bundle over $\mathbf{S}^{4}$. This is the quaternionic Hopf map. $G$-bundles over a four-sphere are classified by an element of $\pi_{3}(G)$. In this case, $\pi_{3}(S U(2)) \cong \mathbb{Z}$ and the transition funtions of the quaternionic Hopf map correspond to the generator of $\pi_{3}(S U(2))$. Moreover, the Euler class of the negative chirality spin bundle $\Sigma_{-} \mathbf{S}^{4}$ is the generator of $H^{4}\left(\mathbf{S}^{4} ; \mathbb{Z}\right) \cong \mathbb{Z}$. The one-parameter family of $\operatorname{Spin}(7)$ metrics on the total space of $\Sigma_{-}$are given explicitly by

$$
d s^{2}=\left(1-\left(\frac{a}{r}\right)^{10 / 3}\right)^{-1} d r^{2}+\frac{9}{100} r^{2}\left(1-\left(\frac{a}{r}\right)^{10 / 3}\right)\left(\sigma_{i}-A^{i}\right)^{2}+\frac{9}{20} r^{2} d \Omega_{4}^{2}
$$

Here, the $\sigma_{i}$ are a set of left-invariant one-forms on $S U(2)$, and the connection $A^{i}$ is the BPST Yang-Mills instanton on the unit four-sphere, whose metric we denote $d \Omega_{4}^{2}$. The Spin(7) metric is complete on $r \geq a$, with $r=a$ being the Cayley $\mathbf{S}^{4}$. At large distance, the metric is asymptotic to the cone over the squashed (weak G2) Einstein seven-sphere.

The construction of this metric has recently been extended in [15]. The idea is simple. In (2.4) the level surfaces are an $\mathbf{S}^{7}$, described as an $\mathbf{S}^{3}$ bundle over $\mathbf{S}^{4}$ with the $\mathbf{S}^{3}$ fibres being "round". One may take a similar ansatz, but this time allow the $\mathbf{S}^{3}$ fibres themselves to become squashed. This allows for the possibility that the $U(1)$ fibres of $U(1) \hookrightarrow \mathbf{S}^{3} \rightarrow \mathbf{S}^{2}$ approach a constant length asymptotically, rather like the Taub-NUT metric. Indeed, this is precisely what happens. The Spin(7) manifold of this form is given by

$d s^{2}=\frac{(r-a)^{2}}{(r-3 a)(r+a)} d r^{2}+a^{2} \frac{(r-3 a)(r+a)}{(r-a)^{2}} \sigma^{2}+\frac{1}{4}(r-3 a)(r+a)\left(D \mu^{i}\right)^{2}+\frac{1}{2}\left(r^{2}-a^{2}\right) d \Omega_{4}^{2}$

The metric $d \Omega_{4}^{2}$ is again the round $\mathbf{S}^{4}$ and, roughly speaking, the one-form $\sigma$ corresponds to the Hopf $U(1)$ fibre over $\mathbf{S}^{2}$, where the $\mathbf{S}^{2}$ has metric $\left(D \mu^{i}\right)^{2}$. The reader is referred to [15] for the precise definitions. As $r \searrow 3 a$, the level surfaces $\mathbf{S}^{7}$ collapse smoothly down to a Cayley $\mathbf{S}^{4}$. Thus the global topology of this space is the same as (2.4). However, there is an important difference between the two. At large radius, the $U(1)$ fibres of (2.5) (parametrised by $\sigma$ ) tend to a constant length as $r$ tends to infinity. 
The remainder of the metric asymptotes to a cone over $\mathbb{C P}^{3}$, with its nearly Kähler Einstein metric (rather than the usual Kähler Fubini-Study metric). Thus this solution is asymptotically locally conical (ALC) rather than asymptotically conical (AC). The new $\operatorname{Spin}(7)$ metric in the present paper (4.54) closely resembles this solution.

We should also point out that the same local solution (2.5) also describes a $\operatorname{Spin}(7)$ metric on $\mathbb{R}^{8}$, simply by taking the range of $r$ to be negative.

Finally, in [24], a $\operatorname{Spin}(7)$ metric was found on a $\mathbb{Z}_{2}$ quotient of the cotangent bundle of $\mathbb{C P}^{2}$. This construction will be explained in detail in section 4 , and the reader should refer back to this section at the appropriate points. The solution in [24] solves the system (4.46) with $k=l=1$

$d s^{2}=\left(1-\left(\frac{a}{r}\right)^{10 / 3}\right)^{-1} d r^{2}+\frac{9 r^{2}}{100}\left(1-\left(\frac{a}{r}\right)^{10 / 3}\right)\left(\lambda^{2}+4 \nu_{1}^{2}+4 \nu_{2}^{2}\right)+\frac{9 r^{2}}{10}\left(\sigma_{1}^{2}+\sigma_{2}^{2}+\Sigma_{1}^{2}+\Sigma_{2}^{2}\right)$

Careful analysis [24] shows that the topology of the three-dimensional fibres with metric $\left(\lambda^{2}+4 \nu_{1}^{2}+4 \nu_{2}^{2}\right)$ is $\mathbb{R} \mathbf{P}^{3}$, which collapse down to $\mathbb{C P}^{2}$ at $r=a$, with metric $\frac{9 a^{2}}{10}\left(\sigma_{1}^{2}+\sigma_{2}^{2}+\Sigma_{1}^{2}+\Sigma_{2}^{2}\right)$. This solution corresponds to viewing the level surfaces $N_{1,1}$ as an $S O(3)=\mathbb{R} \mathbf{P}^{3}$ bundle over $\mathbb{C P}^{2}$. This will be explained in section 4 . The solution is therefore defined on the orbifold $T^{*} \mathbb{C P}^{2} / \mathbb{Z}_{2}$, and is asymptotically conical.

For completeness, we also mention that the ansatz (4.45) contains the hyper-Kähler Calabi metric [25]

$d s^{2}=\left(1-\frac{1}{r^{4}}\right)^{-1} d r^{2}+\frac{1}{4} r^{2}\left(1-\frac{1}{r^{4}}\right) \lambda^{2}+r^{2}\left(\nu_{1}^{2}+\nu_{2}^{2}\right)+\frac{1}{2}\left(r^{2}-1\right)\left(\sigma_{1}^{2}+\sigma_{2}^{2}\right)+\frac{1}{2}\left(r^{2}+1\right)\left(\Sigma_{1}^{2}+\Sigma_{2}^{2}\right)$

which was also explicitly constucted in [24]. The manifold is $T^{*} \mathbb{C P}^{2}$, which corresponds to viewing $N_{1,1}$ as an $\mathbf{S}^{3}$ bundle over $\mathbb{C P}^{2}$. Again, these comments should become transparent later.

\section{Topological Charges And Relation To Singulari- ties Of Calibrated Cycles}

\subsection{Circle Quotients}

As pointed out by Atiyah and Witten [2], one can often view M-theory on a noncompact manifold $X$ of special holonomy as a certain configuration of D-branes in 
a (topologically) Minkowski space. More precisely, given a suitable $U(1)$ action on $X$ such that $X / U(1) \cong \mathbb{R}^{n}$, one can identify this $U(1)$ with the so-called "M-theory circle". Then, the geometry of $X$ is mapped to the geometry of the fixed point set $L$ of the $U(1)$ action. In particular, when $X$ develops a conical singularity, so does $L$. Indeed, if $X$ is a cone on $Y$ such that

$$
Y / U(1) \cong \mathbf{S}^{n-1}
$$

then $L$ is a cone on $F$, where $F$ is the fixed point set in $Y$, in the notations of [2].

Since $Y$ is a smooth closed manifold, the fixed point set $F \subset Y$ of a semi-free $U(1)$ action? on $Y$ is a smooth closed submanifold of even codimension [26, 27]. Consider a fixed point $p \in Y$, where $Y$ is an oriented $n$-manifold equipped with a semi-free circle action, which preserves the orientation. Then the circle group action maps the tangent space $T_{p} Y$ at $p$ into iteself. Hence $T_{p} Y$ is a real $U(1)$-module, which we may decompose into its real irreducible representations. These are either one or two-dimensional:

$$
\begin{aligned}
& \pm 1 \\
& R(\theta)=\left(\begin{array}{cc}
\cos \theta & -\sin \theta \\
\sin \theta & \cos \theta
\end{array}\right)
\end{aligned}
$$

Hence one may decompose the circle action on $T_{p} Y$ into $r 2 \times 2$ rotations with parameters say $\theta_{j}=\kappa_{j} \tau,(j=1, \ldots, r)$, together with $(n-2 r)$ trivial \pm 1 representations. Here, $\tau$ is the $U(1)$ group parameter, and $\kappa_{j}$ are the skew eigenvalues of the matrix $k_{a ; b}$, which are the orthonormal-frame components of the covariant derivative of the Killing covector $k$ associated with the $U(1)$ isometry. The fact that the action preserves the orientation means that we must have an even number of "- 1 "s.

What we have just done is to decompose the tangent space $T_{p} Y$ into directions tangent to the codimension $2 r$ fixed point set $F \subset Y$ containing the point $p \in F$ (these are the trivial "1" representations), and directions normal to $F$. The circle action acts orthogonally on this normal space, and decomposes into $r 2 \times 2$ rotations in $r$ orthogonal 2-planes. Of course, for the orbits to close, the eigenvalues $\left\{\kappa_{j} \mid j=1, \ldots, r\right\}$ must be rationally related. This means that, after rescaling $\tau$ appropriately, the action on the $j^{\text {th }}$ normal 2-plane is by multiplication by $e^{i n_{j} \tau}$, where the integers $n_{j}$ are relatively prime (in order that the action be effective), and $\tau$ has period $2 \pi$.

This action is not semi-free in general. Consider the unit $(2 r-1)$-sphere in the normal space. Its quotient under the circle action gives the so-called weighted projective space $\mathbb{C} \mathbf{P}^{\left[n_{1}, \ldots, n_{r}\right]}$. This is a complex orbifold for general integers $\left\{n_{j}\right\}$. Only if all the $n_{j}= \pm 1$

\footnotetext{
${ }^{3}$ This means that $U(1)$ acts freely on the complement of the fixed point set $F$.
} 
do we get a free action on the sphere, the projection then being the Hopf (or anti-Hopf) map. Thus, a necessary condition for the circle action to be semi-free is that all the integers $n_{j}= \pm 1$, for every connected component of the fixed point set.

If we require the orbit space to be a smooth manifold, $F$ must be either codimension two or four in $Y$. Codimension two corresponds to $r=1$. In this case, the "unit sphere" in the normal space is just a circle. Indeed, we can define polar coordinates $(r, \phi)$ on the normal space. Taking the quotient obviously yields a half-line $\mathbb{R}^{+}$, parametrised by the radial coordinate $r \geq 0$. So, the orbit space looks locally (in a neighbourhood of the fixed point set) like $F \times \mathbb{R}^{+}$. In this way, $F$ becomes a boundary of the orbit space. An illustrative example is a two-dimensional disk, $D^{2} \cong \mathbf{S}^{3} / U(1)$, which may be viewed as a quotient of $\mathbf{S}^{3}$ by a semi-free $U(1)$ action. In this example the $U(1)$ acts freely at a general point on the 3 -sphere, except for the "equator" $F=\mathbf{S}^{1}$, which is clearly a subspace of codimension two in $\mathbf{S}^{3}$.

On the other hand, in the case of codimension four, the orbit space is a smooth closed manifold containing $F$ as a submanifold of codimension three. This corresponds to $r=2$. The unit sphere in the normal space is a 3 -sphere, and the quotient by the $U(1)$ action is the Hopf map. Hence this 3 -sphere projects down to a 2 -sphere in the orbit space. We may now "fill in" this 2-sphere bundle over $F$ with the associated 3-disc bundle over $F$, obtaining a smooth closed manifold which contains $F$.

We do not obtain a smooth orbit space for higher codimension. In particular, when $r=3$, the 5 -sphere in the normal space projects down to a $\mathbb{C P}^{2}$ - which is not the boundary of anything! Since we are interested in the case when $Y / U(1)$ is a homotopy sphere, in particular, when it is a space without a boundary, we should therefore restrict ourselves to the case of codimension four, which is also the most interesting case in physics. Given this motivation, we shall focus on the case when $F$ is codimension four in $Y$, which implies that $L$ is codimension four in $X$.

Semi-free $U(1)$ actions with fixed points of codimension four are very familiar in string theory - they correspond to D6-branes. For instance, $Y=\mathbf{S}^{10}$ admits 8 topologically different $U(1)$ actions with fixed point set being the standard $\mathbf{S}^{6}$, among which there is a semi-free action corresponding to $Y / U(1) \cong \mathbf{S}^{9}$ 28. Building cones on all of these spheres, we find that $X=\mathbb{R}^{11}$ admits a $U(1)$ action such that $X / U(1) \cong$ $C\left(\mathbf{S}^{9}\right)=\mathbb{R}^{10}$, with fixed point set

$$
L \cong C\left(\mathbf{S}^{6}\right)=\mathbb{R}^{7}
$$

This gives a mathematical construction of a flat D6-brane with world volume $L \subset \mathbb{R}^{10}$ as a fixed point set of a $U(1)$ action on the eleven-dimensional space-time $X \cong \mathbb{R}^{11}$.

Now let us implement the fact that $X$ has a reduced holonomy group. This means that there is at least one covariantly constant spinor on $X$ and, therefore, M-theory 
compactification on $X$ is supersymmetric. Hence, the same should be true about the equivalent configuration of D6-branes on $L \subset \mathbb{R}^{10}$ in Type IIA string theory. In general, in such a reduction from M-theory down to Type IIA one does not obtain the standard flat metric on $X / U(1) \cong \mathbb{R}^{n-1}$ due to non-constant dilaton and other fields in the background. However, one would expect that near the singularities of the D-brane locus $L$ these fields exhibit a regular behavior, and the metric on $X / U(1)$ is approximately flat, $c f$. [2]. In this case the condition for the Type IIA background to be supersymmetric can be expressed as a simple geometric criterion: it says that the Dbrane locus $L$ should be a calibrated submanifold in $X / U(1)$ [14, 29, 30, 31, 32, 33, 34].

Even though our discussion in this subsection is quite general, in order to be specific, let us focus on manifolds $X$ of $\operatorname{Spin}(7)$ holonomy - the main theme of this paper. One can easily extend all of the results obtained for $\operatorname{Spin}(7)$ manifolds to other cases, see e.g. (3.46) - 3.47) below.

In the case when an 8-manifold $X$ admits a $\operatorname{Spin}(7)$ structure $\Phi$, then away from the fixed point set $L$ the quotient space $X / U(1)$ has a 3 -form $\Psi=\pi_{*} \Phi$, where $\pi$ is the projection $\pi: X \rightarrow X / U(1)$. The form $\Psi$ defines an "approximate" $G 2$ structure on $X / U(1)$, which becomes a $G 2$ structure in the limit when all $U(1)$ orbits on $X$ have the same length, $c f$. [2]. In this approximation, the fixed point set $L$ representing the location of a D6-brane must be a supersymmetric cycle in $X / U(1)$, namely a coassociative submanifold, calibrated with respect to $\star \Psi$. Therefore, the problem of studying dynamics of M-theory on $\operatorname{Spin}(7)$ singularities can be restated as a problem of studying D6-brane configurations on singular coassociative submanifolds in flat space. When $X$ develops a conical singularity, the D-brane locus $L$ also becomes a (singular) cone on $F \subset Y=\partial X$.

\subsection{Identification of Topological Charges}

The topology of $L$, which determines the dynamics of the D6-branes, can be deduced from the topology of the 8-manifold $X$. In the remainder of this subsection we obtain relations between various homology groups of $X$ and $L$, identifying domain walls and other topologically stable objects in M-theory on $\mathbb{R}^{3} \times X$ and in Type IIA theory with D6-branes on

$$
\mathbb{R}^{3} \times L \subset \mathbb{R}^{10}
$$

For simplicity, let us assume that there is only one D6-brane on every connected component of $L$. Then, from the M-theory perspective, topological charges in the effective $\mathcal{N}=1$ three-dimensional theory correspond to membranes and five-branes wrapped on

\footnotetext{
${ }^{4}$ The part of the D-brane world-volume that is transverse to $X$ is flat and does not play an important rôle in our discussion here.
} 
topologically non-trivial cycles in $X$. On the other hand, in the Type IIA picture with a D6-brane these topological charges are represented by strings and D4-branes which end on the D6-brane.

The fact that only fundamental strings and D4-branes are allowed to have their boundaries on a D6-brane follows from the structure of the Chern-Simons terms on D6-brane world-volumes in Type IIA theory [35]. For example, for a D4-brane this follows from the modified Bianchi identity:

$$
d\left(G^{(4)}-B \wedge G^{(2)}\right)=0
$$

which leads to the following modification of the 4-brane charge:

$$
Q_{4}=\int_{\mathbf{S}^{4}}\left(G^{(4)}-G^{(2)} \wedge B\right)
$$

Now, sliding the 4-sphere to the end of the D4-brane and deforming it into a product $\mathbf{S}^{2} \times \mathbf{S}^{2}$, with the last $\mathbf{S}^{2}$ factor embedded into the D6-brane world-volume, we come to the region where $Q_{4}$ has a contribution mainly from the second term:

$$
Q_{4} \approx-\int_{\mathbf{S}^{2}} G^{(2)} \times \int_{\mathbf{S}^{2}} B=-Q_{6} \times \int_{\mathbf{S}^{2}} B
$$

In a similar way one can show that a D2-brane cannot end on a single D6-brane. Indeed, there is no Chern-Simons term like $\int G^{(4)} \wedge G^{(2)} \wedge C^{(4)}$ in the Type IIA effective action.

This also suggests that the boundary of a D4-brane inside a D6-brane is magnetically charged with respect to the $U(1)$ gauge field on the D-brane world volume. The best way to see this is from the Wess-Zumino term [36]:

$$
I_{\mathrm{WZ}}=\int_{\mathbb{R}^{3} \times L} e^{(\mathcal{F}-B) / 2 \pi} \wedge C_{*}
$$

which includes the term

$$
\int_{\mathbb{R}^{3} \times L} \frac{(\mathcal{F}-B)}{2 \pi} \wedge C_{5}
$$

We will use the fact that the first Chern class of $\mathcal{F}$ jumps once a D4-brane ends on a D6-brane further below.

Now we want to construct various (extended) objects in the effective $\mathcal{N}=1$ threedimensional theory and compare their charges with the corresponding objects in Mtheory. In fact, the correspondence has to be one-to-one, so that the charges computed both ways must be the same. Note that we are not only saying that the charge spectrum 


\begin{tabular}{cll} 
IIA Theory & & M-Theory \\
\hline D4-brane & $\longrightarrow$ & M5-brane \\
String & $\longrightarrow$ & M2-brane
\end{tabular}

Table 2: M-theory lift of a D4-brane and a fundamental string, which are allowed to end on D6-branes in Type IIA string theory.

should be the same, but that every object actually has its counterpart. Namely, a D4brane ending on D6-brane lifts in M-theory to an M5-brane wrapped on a certain cycle $\Sigma \subset X$. Since the D6-brane configuration lifts to a purely geometrical background in M-theory, viz. to the geometry of the space $X$, the cycle $\Sigma$ should be closed in $X$, for otherwise the five-brane would end on "nothing". Similarly, a string ending on a D6-brane lifts to a membrane wrapped on a closed submanifold in $X$. This general rule is summarised in Table 2. We also remark that this argument does not depend on the amount of supersymmetry and, therefore, can be used in compactifications on manifolds of arbitrary holonomy.

Now let us consider specific cases in more detail:

Domain Walls: In Type IIA string theory domain walls correspond to D4-branes with boundary on the D6-brane world-volume, $\mathbb{R}^{3} \times L$. More precisely, the D4-brane world-volume is $\mathbb{R}^{2} \times D^{(3)} \subset \mathbb{R}^{3} \times X / U(1)$, such that:

$$
\partial D^{(3)}=\Sigma^{(2)} \subset L
$$

In order for the D4-brane to be topologically stable, $\left[\Sigma^{(2)}\right]$ should represent a nontrivial homology class in $H_{2}(L ; \mathbb{Z})$. By Poincaré duality, the latter group is isomorphic to $H_{\text {cpct }}^{2}(L ; \mathbb{Z})$, the cohomology with compact support. Therefore, we conclude that in a Type IIA background with a D6-brane, domain walls are classified by the group:

$$
H_{2}(L ; \mathbb{Z}) \cong H_{\text {cpct }}^{2}(L ; \mathbb{Z})
$$

Since D4-branes lift to M5-branes, in M-theory every such domain wall becomes a five-brane with world-volume:

$$
\mathbb{R}^{2} \times \Sigma^{(4)} \subset \mathbb{R}^{3} \times X
$$

Now, $\Sigma^{(4)}$ must be a closed topologically non-trivial 4-cycle in $X$, for otherwise the domain wall would not be stable. Hence, from the M-theory point of view, domain wall charges take values in the group

$$
H_{4}(X ; \mathbb{Z})
$$


Since the spectrum of domain walls should be equivalent in both pictures, we conclude that (3.11) and (3.13) should be isomorphic:

$$
H_{2}(L ; \mathbb{Z}) \cong H_{4}(X ; \mathbb{Z})
$$

Furthermore, as we noted earlier, the first Chern class of the $U(1)$ gauge bundle on the D6-brane in the Type IIA model jumps by the dual cohomology class $\widehat{\left[\Sigma^{(2)}\right]} \in$ $H_{\text {cpct }}^{2}(L ; \mathbb{Z})$ when we cross such a domain wall [37. On the other hand, different vacuum states on a D6-brane configuration are classified by the first Chern class, which takes values in the cohomology group

$$
H^{2}(L ; \mathbb{Z})
$$

There is a natural map:

$$
f: H_{\text {cpct }}^{2}(L ; \mathbb{Z}) \rightarrow H^{2}(L ; \mathbb{Z})
$$

which "forgets" that a cohomology class has compact support. In general, this is not an isomorphism when $L$ is non-compact. In fact, we may write down part of the long exact cohomology sequence for the pair $(L, F)$, where $F=\partial L$ is the boundary "at infinity" in $L$

$$
\ldots H^{2}(L, F ; \mathbb{Z}) \stackrel{f}{\longrightarrow} H^{2}(L ; \mathbb{Z}) \stackrel{i^{*}}{\longrightarrow} H^{2}(F ; \mathbb{Z}) \stackrel{\delta^{*}}{\longrightarrow} H^{3}(L, F ; \mathbb{Z}) \longrightarrow \ldots
$$

Here $i: F \hookrightarrow L$ denotes inclusion, and $H^{2}(L, F ; \mathbb{Z}) \cong H_{\text {cpct }}^{2}(L ; \mathbb{Z})$. By Poincaré duality, $H^{3}(L, F ; \mathbb{Z}) \cong H_{1}(L ; \mathbb{Z})$. Hence, when $L$ is simply-connected, we see that the exact sequence (3.17) implies that different vacua, modulo those connected by domain walls, are classified by the group

$$
H^{2}(F ; \mathbb{Z})=H^{2}(L ; \mathbb{Z}) / f\left(H_{\mathrm{cpct}}^{2}(L ; \mathbb{Z})\right)
$$

In fact, we shall find that $H_{1}(L ; \mathbb{Z})=0$ for the examples in the present paper, so that the above formula applies.

Different vacua in M-theory are classified by the flux of $G$, which in turn is classified (see (3.44)) by the group

$$
H^{4}(X ; \mathbb{Z})
$$

By a similar logic to before, the number of vacua should be the same in the equivalent Type IIA and M-theory models, so that we obtain another useful isomorphism:

$$
H^{2}(L ; \mathbb{Z}) \cong H^{4}(X ; \mathbb{Z})
$$

\footnotetext{
${ }^{5}$ It is actually the shifted $G$-flux, $[G / 2 \pi]-\frac{1}{4} p_{1}(X)$, that may be considered as an integral cohomology class, rather than the $G$-flux itself. This fact will be extremely important in this paper, and will be elaborated on further below.
} 
Now, as in [37, one also finds that different vacua in M-theory, modulo those connected by domain walls, are classified by the group

$$
H^{4}(Y ; \mathbb{Z})=H^{4}(X ; \mathbb{Z}) / f\left(H_{\mathrm{cpct}}^{4}(X ; \mathbb{Z})\right)
$$

where $f$ is again the forgetful map and $Y=\partial X$. More precisely, this formula holds when $H^{5}(X, Y ; \mathbb{Z})=0$. But in the present paper, $X$ is always a four-plane bundle over some four-manifold, $B$. Hence, by the Thom isomorphism, we have

$$
H^{5}(X, Y ; \mathbb{Z}) \cong H^{1}(B ; \mathbb{Z})=0
$$

since $B$ is simply connected (either $B=\mathbf{S}^{4}$ or $B=\mathbb{C P}^{2}$ ). For consistency of both pictures, we must of course have

$$
H^{2}(F ; \mathbb{Z}) \cong H^{4}(Y ; \mathbb{Z})
$$

This is indeed consistent with the formulae (3.18), (3.21), together with the relations between the homology and cohomology groups of $L$ and $X$ derived so far in this section.

Finally, we note that all 8-manifolds $X$ with $\operatorname{Spin}(7)$ holonomy that we consider in this paper are simply-connected. (Also, all compact Spin(7)-manifolds are simplyconnected). Therefore, for these manifolds there are no other domain walls, in particular, there are no domain walls constructed from M2-branes. In the Type IIA theory such a domain wall, if it existed, would look like a D2-brane with boundary on a D6brane. In the case of multiple D6-branes this configuration would be possible, and the boundary of a D2-brane would couple to the second Chern class of the gauge bundle on the D6-branes.

Stable Particles: Again, we start in Type IIA theory with a D6-brane, where stable particles in the three-dimensional effective field theory correspond to either a string or a D4-brane with boundary on a D6-brane.

The case of a D4-brane is very similar to what we considered above. Namely, in order to represent a codimension two object in $2+1$ dimensions, a D4-brane must have world-volume $\mathbb{R}^{1} \times D^{(4)} \subset \mathbb{R}^{3} \times X / U(1)$ such that:

$$
\partial D^{(4)}=\Sigma^{(3)} \subset L
$$

And, following the above arguments, we conclude that topologically stable particles are classified by the homology group

$$
H_{3}(L ; \mathbb{Z})
$$


Since a D4-brane on a 4-manifold $D^{(4)} \subset X / U(1)$ lifts to a five-brane on a closed 5-cycle $\Sigma^{(5)} \subset X$, in M-theory such particles are classified by the group:

$$
H_{5}(X ; \mathbb{Z})
$$

Identifying these objects particle-by-particle in Type IIA and in M-theory, we conclude that (3.25) and (3.26) are isomorphic:

$$
H_{3}(L ; \mathbb{Z}) \cong H_{5}(X ; \mathbb{Z})
$$

There can be another kind of point-like stable object, which in Type IIA corresponds to an open fundamental string ending on a D6-brane. Its world-volume looks like the product of a "time direction" and an interval in space. Particles of this type can be stable only if the ends of the string belong to different connected components of the D6-brane locus, $L$. Therefore, the charges of these particles form a lattice of dimension $h_{0}(L)-1$.

In M-theory, every fundamental string ending on a D6-brane lifts to a closed membrane, wrapped on a 2-cycle $\Sigma^{(2)} \subset X$. The dimension of these states is clearly $h_{2}(X)$, and must be the same as the dimension of the corresponding stable particles in Type IIA theory:

$$
h_{0}(L)-1=h_{2}(X)
$$

Space-Filling Branes: Having established relations between $H_{i}(L ; \mathbb{Z})$, for $i=$ $0,2,3$, and the corresponding homology groups of the 8-manifold $X$, now we have to find a similar formula for $H_{1}(L ; \mathbb{Z})$. We can obtain such a formula, for example, by looking at D4-branes filling three-dimensional space-time ?. They have world-volume $\mathbb{R}^{3} \times D^{(2)} \subset \mathbb{R}^{3} \times X / U(1)$, where

$$
\partial D^{(2)}=\Sigma^{(1)} \subset L
$$

Hence, the charges of such space-filling D4-branes take values in:

$$
H_{1}(L ; \mathbb{Z})
$$

In M-theory, they lift to an M5-brane with world-volume $\mathbb{R}^{3} \times \Sigma^{(3)} \subset \mathbb{R}^{3} \times X$ with $\Sigma^{(3)}$ a closed 3-cycle in $X$. It follows that in M-theory the charges of the corresponding space-filling branes take values in the group

$$
H_{3}(X ; \mathbb{Z})
$$

\footnotetext{
${ }^{6}$ There are other types of space-filling branes, e.g. D2-branes, but they do not lead to new information about the topology of $L$.
} 
Identifying the charges, as before, we find the last isomorphism:

$$
H_{1}(L ; \mathbb{Z}) \cong H_{3}(X ; \mathbb{Z})
$$

As we explain below, these groups are trivial in our models. Hence the only spacefilling branes which can occur are D2-branes/membranes.

Instantons: Instanton effects play a very important role in the effective $\mathcal{N}=1$ three-dimensional gauge theory and will be discussed further below. Here we just mention that they can come either from string or D4-brane instantons which are completely embedded in $X$ and have boundary on the D6-brane locus, $L$. Since $L$ is non-compact, we have to consider only world-sheet instantons.

The world-sheet string instantons with boundary on $L$ are classified by?:

$$
H_{1}(L ; \mathbb{Z})
$$

In M-theory these states correspond to membrane instantons, classified by $H_{3}(X ; \mathbb{Z})$. Therefore, lifting string instantons to M-theory we find a relation between the corresponding homology groups that we have seen in the previous example:

$$
H_{1}(L ; \mathbb{Z}) \cong H_{3}(X ; \mathbb{Z})
$$

In the case of compactification on a Calabi-Yau manifold or a manifold of $G 2$ holonomy this would be the end of story. However, if the dimension of $X$ is greater than or equal to eight, we can discover a world-sheet anomaly related to the fact that $L$ may fail to be a spin manifold [38]. In order to see the anomaly, we start with a string worldsheet, $\Sigma^{(2)}$, with boundary on the D6-brane, and consider a one-parameter deformation of $\Sigma^{(2)}$ along a closed loop $\mathbf{S}^{1}$, such that we have an embedding

$$
\phi: \Sigma^{(2)} \times \mathbf{S}^{1} \rightarrow \mathbb{R}^{7}, \quad \phi\left(\partial \Sigma^{(2)} \times \mathbf{S}^{1}\right) \subset L
$$

Then, going around the loop $\mathbf{S}^{1}$, the string world-sheet path integral picks up a phase factor [38]:

$$
\operatorname{pfaff}(D) \rightarrow(-1)^{\alpha} \cdot \operatorname{pfaff}(D)
$$

where

$$
\alpha=\left(\int_{\partial \Sigma^{(2)} \times \mathbf{S}^{1}} w_{2}(L)\right)
$$

\footnotetext{
${ }^{7}$ The reason we have $H_{1}(L ; \mathbb{Z})$, rather than $\pi_{1}(L)$ is that the 'missing' elements of $\pi_{1}(L)$, which map to the zero element in $H_{1}(L ; \mathbb{Z})$, correspond to bound states of multiple membrane instantons [39]. In this section we restrict ourselves only to basic instantons.
} 
$w_{2}(L)$ is the second Stiefel-Whitney class of $L$, and $\operatorname{pfaff}(D)$ is the Pfaffian of the world-sheet Dirac operator $D$. All oriented manifolds $L$ of dimension less than four are spin, so that $w_{2}(L)=0$ automatically. However, in dimension four, which is relevant to the present paper, one can have a non-trivial class $w_{2}(L) \in H^{2}\left(L ; \mathbb{Z}_{2}\right)$. As we shall see, this is precisely the case for the two $\operatorname{Spin}(7)$ models discussed in the present paper.

If $w_{2}(L)$ happens to be non-zero, one can still have a consistent D6-brane configuration, but in order to achieve this one needs to turn on a non-trivial $U(1)$ "gauge field" on the D6-brane world-volume:

$$
\operatorname{pfaff}(D) \cdot \exp \left(i \oint_{\partial \Sigma^{(2)}} \mathcal{A}\right)
$$

Then, on going around the loop $\mathbf{S}^{1}$ the phases of the two factors can compensate each other if the field strength $\mathcal{F}$ obeys the modified quantisation condition:

$$
\left[\frac{\mathcal{F}}{2 \pi}\right]-\frac{\nu}{2} \in H^{2}(L ; \mathbb{Z})
$$

Here $r(\nu)=w_{2}(L)$, under the reduction modulo two homomorphism $r: H^{2}(L ; \mathbb{Z}) \rightarrow$ $H^{2}\left(L ; \mathbb{Z}_{2}\right)$.

Now let us consider what happens when we lift this configuration to M-theory. As we mentioned earlier, a string becomes a membrane wrapped over a closed 3-cycle $\Sigma^{(3)} \subset X$. The world-sheet fermion anomaly lifts to the membrane anomaly in Mtheory. Specifically, the way we detected the anomaly in Type IIA was by looking at a one-parameter family of string world-sheets parametrised by a circle $\mathbf{S}^{1}$. In Mtheory, $\Sigma^{(2)}$ lifts to $\Sigma^{(3)}$, so that the process described above corresponds to studying a one-parameter family of closed 3-cycles, such that

$$
\Sigma^{(3)} \times \mathbf{S}^{1} \subset X
$$

And, again, there are two dangerous factors in path integral in the membrane worldvolume theory, corresponding to the Pfaffian of the Dirac operator and to the period of the $C$-field. After going around the closed loop $\mathbf{S}^{1}$ these two factors pick up a phase [18]:

$$
\exp \left(i \int_{\Sigma^{(3)} \times \mathbf{S}^{1}} \pi \lambda+G\right)
$$

where the integral class $\lambda=p_{1}(X) / 2 \in H^{4}(X ; \mathbb{Z})$ is canonically defined for a spin manifold $X$, since $p_{1}(X)$ is always divisible by two in this case 2 . The definition is very similar to the above definition of the class $\nu$, but with $w_{2}(L)$ replaced by $w_{4}(X)$. Specifically, $\lambda$ is congruent modulo two to $w_{4}(X)$. Note also that both $\nu$ and $\lambda$ come

\footnotetext{
${ }^{8}$ More fundamentally, $\lambda$ is the first obstruction class to the spin bundle of $X$ [0].
} 
from fermionic anomalies in the string/membrane world-volume theory, so that it is natural to identify the two. More precisely, we can express this as a map:

$$
\rho: \lambda \mapsto \nu
$$

under the isomorphism:

$$
\rho: H^{4}(X ; \mathbb{Z}) \rightarrow H^{2}(L ; \mathbb{Z})
$$

As evidence for the proposed identification, let's see what happens if $\lambda / 2$ is not an integral class. In this case, the $G$-field in M-theory has to obey a shifted quantization condition [18]:

$$
\left[\frac{G}{2 \pi}\right]-\frac{\lambda}{2} \in H^{4}(X ; \mathbb{Z})
$$

similar to the $\mathcal{F}$-field quantisation condition (3.39). For example, when $\lambda$ (respectively $\nu$ ) is odd, we need to turn on a half-integral $G$ (respectively $\mathcal{F}$ ) flux. In particular, as expected both models are (non-)anomalous at the same time. This agrees with our general identification of degree two cohomology elements in $L$ and degree four cohomology elements in $X$.

Altogether, we may summarise all of the above relations as follows:

$$
\begin{aligned}
h_{0}(L) & =h_{2}(X)+1 \\
H_{i}(L ; \mathbb{Z}) & \cong H_{i+2}(X ; \mathbb{Z}), \quad i>0 \\
H^{2}(L ; \mathbb{Z}) & \cong H^{4}(X ; \mathbb{Z}) \\
\nu & \stackrel{\rho}{=} \lambda
\end{aligned}
$$

Applying similar arguments to other manifolds of special holonomy, such as G2manifolds or Calabi-Yau manifolds, one can obtain the same universal result:

$$
H_{i}(L ; \mathbb{Z})=H_{i+2}(X ; \mathbb{Z}), \quad 0<i<\operatorname{dim}_{\mathbb{R}}(L)
$$

This general formula is valid for all $i$, except for the special case $i=0$ when

$$
h_{0}(L)=h_{2}(X)+1
$$

Note, for example, that all of the D6-brane geometries dual to $G 2$ conical singularities, studied recently in [2], satisfy this relation.

Example 1: In order to demonstrate how the above ideas work in practice, let's take a $\operatorname{Spin}(7)$ metric (2.4) on $X=\Sigma_{-} \mathbf{S}^{4}$, the total space of the negative chirality spinor bundle over $\mathbf{S}^{4}$. At large distance this space looks like a cone over $Y$, where $Y$ is a 7 -sphere. In this case it follows from Smith Theory that the fixed point set $F$ 
under a semi-free circle action is an integral homology 3-sphere in $Y$. More precisely, $F$ is a knotted homology 3 -sphere [ [28], so that when we build a cone over $F$, we obtain a singular space $L$. Therefore, we conclude that M-theory on a $\operatorname{Spin}(7)$ manifold $X=\Sigma_{-} \mathbf{S}^{4}$ developing a conical singularity is equivalent to Type IIA string theory on $\mathbb{R}^{10} \cong \mathbb{R}^{3} \times X / U(1)$ with a (singular) configuration of D6-branes on

$$
\mathbb{R}^{3} \times L \subset \mathbb{R}^{10}
$$

The space $L$, representing the locus of the D6-brane, may be deformed to a smooth four-manifold which is a non-trivial $\mathbb{R}^{2}$ bundle over $\mathbf{S}^{2}$ :

$$
L \cong \mathbb{R}^{2} \times \mathbf{S}^{2}
$$

Specifically, this bundle is the spin bundle of $\mathbb{C P}^{1} \cong \mathbf{S}^{2}$, which has first Chern class (or equivalently Euler class) given by the generator of $H^{2}\left(\mathbf{S}^{2} ; \mathbb{Z}\right) \cong \mathbb{Z}$. That is, we have effectively blown up the origin of $\mathbb{R}^{4}$ with a copy of $\mathbb{C} \mathbf{P}^{1}$. In fact, it is not hard to construct explicitly the $U(1)$ action on $X$ such that the fixed point set is $L$ above, with an orbit space that may be given the differentiable structure of $\mathbb{R}^{7}$. Since we will not require the details of this in the present paper, we shall leave the precise description for a future publication [20].

We may now use this example to verify the formulae (3.45) in this particular case. The only non-trivial homology groups of $X$ are in dimension 0 and 4 :

$$
H_{0}(X ; \mathbb{Z})=H_{4}(X ; \mathbb{Z})=\mathbb{Z}
$$

Therefore, according to the above discussion, the non-trivial homology groups of $L$ must be

$$
H_{0}(L ; \mathbb{Z})=H_{2}(L ; \mathbb{Z})=\mathbb{Z}
$$

Clearly this is the case for $L$ of the form (3.49). This example therefore confirms the relations between the homology groups of $X$ and $L$ found in the general analysis above. Notice also that, as expected in this example, $L$ is not spin. As explained above, this is precisely because $\lambda(X)$ is not divisible by two.

Example 2: We can also consider $X$ to be an $\mathbb{R}^{4}$ bundle over $\mathbb{C P}^{2}$, corresponding to the M-theory lift of a D6-brane wrapped on the coassociative cycle of the $G 2$-space $\Lambda^{-} \mathbb{C P}^{2}$. The complete metrics of $\operatorname{Spin}(7)$ holonomy on different bundles of this kind will be discussed in the next section. In all cases we have

$$
H_{i}(X ; \mathbb{Z})= \begin{cases}\mathbb{Z} & i=0,2,4 \\ 0 & \text { otherwise }\end{cases}
$$

\footnotetext{
${ }^{9}$ Such 3-spheres are related to the ordinary knots in $\mathbf{S}^{3}$. It would be interesting to understand the meaning of these knots in the $\mathcal{N}=1$ three-dimensional effective theory with Chern-Simons term that we discuss in section 6 .
} 
Now consider a reduction from M-theory on $X$ to Type IIA string theory on $\mathbb{R}^{10}$ with D6-branes wrapped on $L \subset X / U(1) \cong \mathbb{R}^{10}$. From the identification of topologically stable objects it follows that $L$ has non-trivial homology groups

$$
H_{0}(L ; \mathbb{Z})=\mathbb{Z} \oplus \mathbb{Z}, \quad H_{2}(L ; \mathbb{Z})=\mathbb{Z}
$$

This agrees with the general result of [27, 28, [1] that $F$ has to be a codimension 4 subspace of $Y=S U(3) / U(1)$. In fact, a typical $U(1) \subset S U(3)$ has a fixed point set $\{$ pt $\} \times \mathbb{C} \mathbf{P}^{1}$ inside $\mathbb{C} \mathbf{P}^{2}$. Since $Y$ may be viewed as a homotopy 3 -sphere bundle over $\mathbb{C P}^{2}$ it follows that the fixed point set must be the union of a typical fibre and a circle bundle over $\mathbf{S}^{2}$. In this example we find that

$$
L=\mathbb{R}^{4} \cup \mathbb{R}^{2} \times \mathbf{S}^{2}
$$

which indeed has the homology groups (3.53). Again, the second factor is more precisely given by the total space of the spin bundle of $\mathbf{S}^{2}[20]$.

\section{New Complete Non-Compact Spin(7) Manifolds}

In this section we present a new one-parameter family of complete metrics on the universal quotient bundle $\mathcal{Q}$ of $\mathbb{C P}^{2}$. The method we use is a generalisation of the procedure recently used to construct the hyper-Kähler Calabi metrics on $T^{*} \mathbb{C} P^{n}$, the cotangent bundle of $\mathbb{C P}^{n}$ [24]. In fact, the authors of that paper also found a system of first order equations describing $\operatorname{Spin}(7)$ metrics, and presented the solution (2.6) which lives on a $\mathbb{Z}_{2}$ orbifold of the total space of $T^{*} \mathbb{C} \mathbf{P}^{2}$. As in section 2 , one again assumes a cohomogeneity one ansatz, with level surfaces this time taken to be the coset space $S U(3) / U(1)$. There are an infinite number of distinct ways of embedding the $U(1)$ in $S U(3)$, but the solutions in [24] are described by the same embedding. The new $\operatorname{Spin}(7)$ metrics presented in this section correspond to a different embedding of the circle $U(1)$ in the group manifold $S U(3)$. Specifically, the manifold on which the new family of $\operatorname{Spin}(7)$ metrics is defined is the total space of the universal quotient bundle $\mathcal{Q}$.

The new $\operatorname{Spin}(7)$ metrics we have found here have recently been generalised in [16], the results of which will be summarised below. Their local solutions that extend our solution (4.54), together with the global analysis of the next subsection, are complementary. The upshot is that we obtain a set of $\operatorname{Spin}(7)$ metrics defined on the total space of various cyclic quotients of any $\operatorname{spin}^{c}$ structure over $\mathbb{C} \mathbf{P}^{2}$. We would like to thank the authors of [16] for sharing their results with us prior to publication. 


\subsection{Aloff-Wallach Spaces}

We begin then with a discussion of the coset space $S U(3) / U(1)$. These so-called AloffWallach spaces were studied by various authors [42], mainly in the context of finding new Einstein manifolds. We extend this work considerably, elucidating in particular the global topology, and also make a connection between Aloff-Wallach spaces and the space of $\operatorname{spin}^{c}$ structures over $\mathbb{C P}^{2}$. These results therefore describe the global topology of the local solutions found recently in [16].

Let $G=S U(3)$. Every non-trivial circle in $G$ is of the form

$$
T_{k, l}: e^{2 \pi i t} \rightarrow\left\{\left[\begin{array}{ccc}
e^{2 \pi i k t} & 0 & 0 \\
0 & e^{2 \pi i l t} & 0 \\
0 & 0 & e^{-2 \pi i(k+l) t}
\end{array}\right] \mid t \in \mathbb{R} / \mathbb{Z}\right\}
$$

up to conjugation in $S U(3)$, where the integers $k$ and $l$ are not both zero. For the action to be effective, which we assume, we require that $k$ and $l$ be relatively prime, $\operatorname{gcd}(k, l)=1$. Then, by definition, the Aloff-Wallach space $N_{k, l}$ is the quotient space

$$
N_{k, l} \equiv S U(3) / U(1)=G / T_{k, l}
$$

It will be necessary to consider various subgroups of $S U(3)$. In particular, we define

$$
H=\left\{\left[\begin{array}{cc}
h & 0 \\
0 & \operatorname{det} h^{-1}
\end{array}\right] \mid h \in U(2)\right\} \cong U(2)
$$

so thatto

$$
\mathbb{C P}^{2}=S U(3) / U(2)=G / H
$$

We also let $T^{2}=U(1) \times U(1) \subset H \subset S U(3)$ be a maximal torus in $S U(3)$. Note that any two maximal tori are conjugate.

Various $N_{k, l}$ spaces are related by conjugation in $S U(3)$. Specifically, it is the Weyl group of $S U(3)$ that permutes various Aloff-Wallach representations of the same manifold. Recall that for any compact connected Lie group $G$ the Weyl group may be defined as the centraliser of the maximal torus. For $G=S U(3)$, the maximal torus is $T^{2}$, and its centraliser is $\Sigma_{3}$, the group of permutations of three elements. The embedding of this group into $S U(3)$ is defined by the group of permutation matrices in $S U(3)$. For example, the element

\footnotetext{
${ }^{10}$ Note that it is $S U(3) / \mathbb{Z}_{3}$ and not $S U(3)$ that acts effectively here.
} 


$$
w_{(2)} \equiv-\left[\begin{array}{lll}
0 & 1 & 0 \\
1 & 0 & 0 \\
0 & 0 & 1
\end{array}\right] \in \Sigma_{3} \subset S U(3)
$$

has order two in $\Sigma_{3}$ and one may easily verify that

$$
w_{(2)}^{-1} \cdot T_{k, l} \cdot w_{(2)}=T_{l, k}
$$

Thus $w_{(2)} \in \Sigma_{3}$ maps $T_{k, l} \mapsto T_{l, k}$. Similarly, one can construct analogous order two elements that map $T_{k, l}$ to $T_{k,-k-l}$ and $T_{-l-k, l}$, respectively. There is also an element of order three, which one may take to be

$$
w_{(3)} \equiv\left[\begin{array}{lll}
0 & 1 & 0 \\
0 & 0 & 1 \\
1 & 0 & 0
\end{array}\right] \in \Sigma_{3} \subset S U(3)
$$

and generates the group of even permutations $\mathbb{Z}_{3} \subset \Sigma_{3}$. Applying $w_{(3)}$ repeatedly one finds

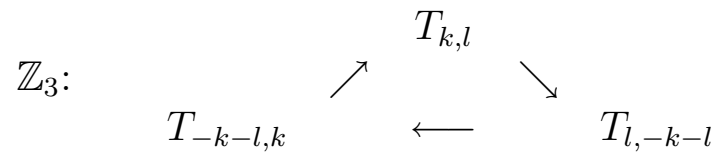

The action of the Weyl group therefore permutes equivalent Aloff-Wallach spaces. Notice that the equivalence class of $N_{1,1}$ is in some sense degenerate, since $w_{(2)}$ maps $N_{1,1}$ into the same representation.

There is also a $\Sigma_{2}=\mathbb{Z}_{2}$ group of outer automorphisms that commutes with $\Sigma_{3}$ and acts by complex conjugation on $S U(3)$ (inducing complex conjugation on $\mathbb{C P}^{2}$ ). On the Aloff-Wallach space $N_{k, l}$ this group action induces an isomorphism:

$$
\Sigma_{2}: \quad N_{k, l} \cong N_{-k,-l}
$$

for every $k$ and $l$. The actions of $\Sigma_{2}$ and $\Sigma_{3}$ are independent, except for the equivalence class of $N_{1,-1}$. In this case, the action of complex conjugation introduces no new representations. For example, the generator of $\Sigma_{2}$ acts in the same way as $w_{(2)}$ on $N_{1,-1}$.

Since the equivalence classes of $N_{1,1}$ and $N_{1,-1}$ are exceptional, we refer to these as the exceptional Aloff-Wallach spaces. The fact that the exceptional Aloff-Wallach spaces behave differently under the symmetry groups will also show up in the geometry, as we shall see later. 
Consider the following tautological bundle diagram

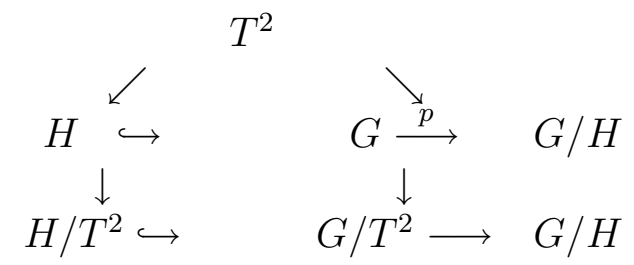

The nested sequence $T_{k, l} \subset T^{2} \subset H \subset G$ allows us to factor through the diagram by $T_{k, l}$

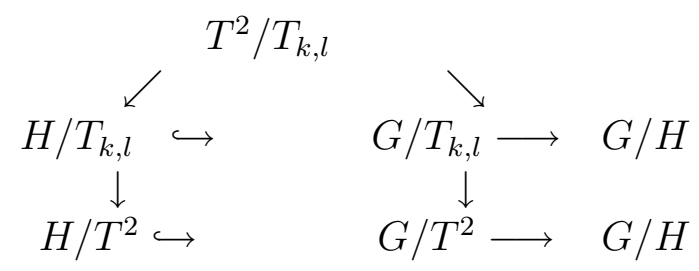

More concretely, this diagram reads

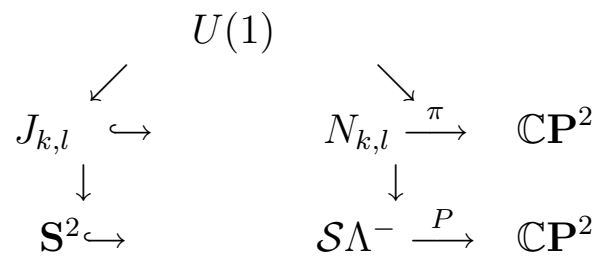

where $\mathcal{S} \Lambda^{-}$is the twistor space of $\mathbb{C} \mathbf{P}^{2}$, and we have defined the quotient space $J_{k, l}=H / T_{k, l}$. Let us examine the diagram (4.12) in more detail.

Reading down the first column, we see that $J_{k, l}$ may be viewed as the total space of a $U(1)$ bundle over $\mathbf{S}^{2}$. Such bundles are in 1-1 correspondence with the first Chern number $\mathbb{Z} \cong H^{2}\left(\mathbf{S}^{2} ; \mathbb{Z}\right)$, the total space then being the Lens space $L(1, N)=\mathbf{S}^{3} / \mathbb{Z}_{N}$ for $N \in \mathbb{Z}^{+}$. Changing the sign of $N$ simply reverses the orientation. When the first Chern class is zero, one has the trivial bundle $\mathbf{S}^{1} \times \mathbf{S}^{2}$, which, for convenience, we define to be the Lens space $L(1,0)$. Hence the quotient manifold $J_{k, l}$ is a Lens space.

The second column says that $N_{k, l}$ may be viewed as a $U(1)$ bundle over the twistor space of $\mathbb{C P}^{2}$. This fact will be extremely important in determining the relation between Aloff-Wallach spaces and the space of $\operatorname{spin}^{c}$ structures on $\mathbb{C P}^{2}$.

The second row of course describes the twistor space as the total space of an $\mathbf{S}^{2}$ bundle over $\mathbb{C P}^{2}$, with projection map $P: \mathcal{S} \Lambda^{-} \rightarrow \mathbb{C P}^{2}$.

Finally, the first row says that $N_{k, l}$ may also be considered as a bundle over $\mathbb{C P}^{2}$ with fibre $J_{k, l}=H / T_{k, l}$ and structure group $H \cong U(2)$. More precisely, $\pi$ is the associated bundle, with fibre $H / T_{k, l}$, to the $H$-principal bundle $p: G \rightarrow G / H$. Thus the Aloff-Wallach space $N_{k, l}$ may be viewed as a Lens space bundle over $\mathbb{C P}^{2}$. 
Indeed, by conjugation in $S U(3)$, each $N_{k, l}$ may be viewed as various different Lens space bundles over $\mathbb{C} \mathbf{P}^{2}$. We would like to understand precisely which Lens spaces occur. To see this, note that the embedding of $T_{p, q} \cong U(1)$ in $H \cong U(2)$ is defined by

$$
e^{2 \pi i t} \rightarrow\left\{\left[\begin{array}{cc}
e^{2 \pi i p t} & 0 \\
0 & e^{2 \pi i q t}
\end{array}\right] \subset U(2) \mid t \in \mathbb{R} / \mathbb{Z}\right\}
$$

Now apply $S$ to the quotient $U(2) / T_{k, l}$. This gives $S U(2) / S\left(T_{p, q}\right)$. Now of course $S U(1)$ is trivial, but the $S$ here refers to the $U(2)$ determinant. Clearly

$$
\operatorname{det}\left[\begin{array}{cc}
e^{2 \pi i p t} & 0 \\
0 & e^{2 \pi i q t}
\end{array}\right]=e^{2 \pi i(p+q) t}
$$

and hence the subgroup of $T_{p, q}=U(1) \subset U(2)$ consisting of matrices with unit determinant is given by $\{r /|p+q||0 \leq r<| p+q \mid, r \in \mathbb{Z}\} \cong \mathbb{Z}_{|p+q|} \subset U(1)$. Thus

$$
J_{p, q}=U(2) / T_{p, q}=S U(2) / S\left(T_{p, q}\right)=\mathbf{S}^{3} / \mathbb{Z}_{|p+q|}=L(1,|p+q|)
$$

In order to specify an Aloff-Wallach space, one needs to give two (relatively prime) integers $k$ and $l$. However, in some sense it is more natural to give a triple $[k, l,-(k+l)]$. The manifold $N_{k, l}$ may then be viewed as an $L(1, N)$ bundle over $\mathbb{C P}^{2}$, where

$$
N=|k|,|l|, \text { or }|k+l|
$$

the choices being permuted by the Weyl group $\Sigma_{3}$.

The reader should now find that some of the remarks at the end of section 2 have become more transparent. A generic $N_{k, l}$ space may be viewed as three different Lens space bundles over $\mathbb{C P}^{2}$ (4.16), permuted by the cyclic group (4.8), whereas the exceptional cases have only two bundle structures. Specifically, $N_{1,1}$ may be viewed as both an $L(1,1)=\mathbf{S}^{3}$ and an $L(1,2)=\mathbf{S}^{3} / \mathbb{Z}^{2}=\mathbb{R} \mathbf{P}^{3}$ bundle over $\mathbb{C} \mathbf{P}^{2}$. The $\operatorname{Spin}(7)$ metric (2.6) on $T^{*} \mathbb{C P}^{2} / \mathbb{Z}_{2}$ corresponds to the latter case, whereas the former case describes the hyper-Kähler Calabi metric (2.7).

Similarly, the space $N_{1,-1}$ may be viewed as an $L(1,1)=\mathbf{S}^{3}$ and an $L(1,0)=\mathbf{S}^{1} \times \mathbf{S}^{2}$ bundle over $\mathbb{C P}^{2}$. The former case is relevant to the new $\operatorname{Spin}(7)$ metrics on the universal quotient bundle $\mathcal{Q}$.

Finally, for future reference, we note that the fourth cohomology group of $N_{k, l}$ is known [42] to be

$$
H^{4}\left(N_{k, l} ; \mathbb{Z}\right) \cong \mathbb{Z}_{r}
$$

where $r=\left|k^{2}+l^{2}+k l\right|$. 


\subsection{The Relation to $\operatorname{Spin}^{\mathrm{c}}$ Structures}

In the construction of new special holonomy metrics, the Aloff-Wallach spaces play the rôle of the level surfaces of a cohomogeneity one metric. As we have just demonstrated, each Aloff-Wallach space may be viewed as various circle bundles over the twistor space of $\mathbb{C P}^{2}$ and also as various Lens space bundles over $\mathbb{C} P^{2}$, related by the action of $\Sigma_{3}$. The Lens space bundles correspond to the boundary of an $\mathbb{R}^{4} / \mathbb{Z}_{N}$ bundle over $\mathbb{C P}^{2}$, which we get by "filling in" the Lens space $L(1, N)$ with $\mathbb{R}^{4} / \mathbb{Z}_{N}$ on each fibre. We would like to understand precisely which $\mathbb{R}^{4}$ bundles arise in this way. In order to do so, we will first of all need to recall some facts about $\operatorname{spin}^{c}$ structures.

Locally, one may always lift an $S O(n)$ bundle (or equivalently an oriented $n$-plane bundle) to its double cover, $\operatorname{Spin}(n)$. However, in general there is a global obstruction to doing this, measured by a certain mod two cohomology class called the second StiefelWhitney class. When this class vanishes, the bundle is said to admit a spin structure. In the case of $\mathbb{C P}^{2}$, one finds that the second Stiefel-Whitney class $w_{2}=w_{2}\left(T \mathbb{C P}^{2}\right)$ of the tangent bundle is the generator of $H^{2}\left(\mathbb{C P}^{2} ; \mathbb{Z}_{2}\right) \cong \mathbb{Z}_{2}$, and hence there is a global obstruction to lifting the tangent bundle $S O(4) \rightarrow \operatorname{Spin}(4) \cong S U(2) \times S U(2)$. Consequently, one may only define the spin bundle $\Sigma=\Sigma_{+} \oplus \Sigma_{-}$locally.

However, for a four-manifold $B$, there is always a complex line bundle $L \rightarrow B$ over $B$ with first Chern class $c_{1}=c_{1}(L) \in H^{2}(B ; \mathbb{Z})$ such that $c_{1}$ reduces to $w_{2}(E)$, modulo two, for any oriented vector bundle $E$. For example, in the case of the tangent bundle, one may formally construct the rank-two complex vector bundles

$$
\mathbb{V}_{ \pm}=\mathbb{V}_{ \pm}(L)=\Sigma_{ \pm} \otimes L^{1 / 2}
$$

These are known as $\operatorname{spin}^{c}$ bundles. The point is that the sign problems that one encounters in trying to consistently define the transition functions of the spin bundles $\Sigma_{ \pm}$are precisely cancelled by the ambiguity in taking the square roots of the transition functions of $L$.

Of course, the choice of $L$ is not unique here; we are free to tensor $\mathbb{V}_{ \pm}$with any complex line bundle $M$ to obtain another $\operatorname{spin}^{c}$ bundle. This generates a free transitive action of the group $H^{2}(B ; \mathbb{Z})$ on the space of $\operatorname{spin}^{c}$ structures. In particular, tensoring $\mathbb{V}_{ \pm}(L)$ with $M$ shifts the first Chern class $c_{1} \equiv c_{1}(L)=c_{1}\left(\mathbb{V}_{ \pm}\right)$by

$$
c_{1} \rightarrow c_{1}+2 a
$$

where $a=c_{1}(M)$. If $H^{2}(B ; \mathbb{Z})$ is torsion-free (as is the case for $B=\mathbb{C P}^{2}$ ), then the space of $\operatorname{spin}^{c}$ structures is in 1-1 correspondence with the set of cohomology classes that reduce modulo two to the second Stiefel-Whitney class. Specialising this discussion 
to the case $B=\mathbb{C} \mathbf{P}^{2}$, we see that the space of $\operatorname{spin}^{c}$ structures on $\mathbb{C} \mathbf{P}^{2}$ correspond to the "odd" classes in $H^{2}\left(\mathbb{C P}^{2} ; \mathbb{Z}\right) \cong \mathbb{Z}$.

What are some of these $\operatorname{spin}^{c}$ structures? First, note that for any Kähler manifold $B$, there is a canonical choice for $L$, and therefore a canonical choice of $\operatorname{spin}^{c}$ structure. Namely, one may take $L=K^{-1}$, where $K=\Lambda^{2,0} B$ is the canonical line bundle, and $\Lambda^{p, 0} B$ denotes the bundle of holomorphic $p$-forms over $B$. The first Chern class $c_{1}(K)$ is then also the first Chern class of $B$. One finds that

$$
\begin{array}{r}
\mathbb{V}_{+}\left(K^{-1}\right)=\Lambda^{0,0} \oplus \Lambda^{0,2}=1 \oplus K^{-1} \\
\mathbb{V}_{-}\left(K^{-1}\right)=\Lambda^{0,1}=T^{+}
\end{array}
$$

where in the last line $T^{+}=T^{+} B$ denotes the holomorphic tangent bundle of $B$. For future reference, in the case of $B=\mathbb{C P}^{2}$, we note that the total Chern class of this bundle is $c\left(T^{+}\right)=(1+x)^{3}=1+3 x+3 x^{2}$ where $x$ generates the cohomology ring of $\mathbb{C} \mathbf{P}^{2}$. In particular, the Euler number of $T \mathbb{C} \mathbf{P}^{2}$ is $3 x^{2}\left[\mathbb{C} \mathbf{P}^{2}\right]=3$.

Instead, we may now tensor the canonical $\operatorname{spin}^{c}$ structure with the line bundle $M$ whose first Chern class is $-x$. We find in particular that

$$
\mathbb{V}_{-}=\mathcal{Q}
$$

is the universal quotient bundle of $\mathbb{C P}^{2}$. This is defined as follows 43. Consider the tautological exact sequence over $\mathbb{C} \mathbf{P}^{2}$

$$
0 \rightarrow S \rightarrow \mathbb{C P}^{2} \times \mathbb{C}^{3} \rightarrow \mathcal{Q} \rightarrow 0
$$

Here, $S$ is the universal subbundle, defined as

$$
S=\left\{(l, z) \in \mathbb{C P}^{2} \times \mathbb{C}^{3} \mid z \in l\right\}
$$

That is, the fibre of $S$ above the point $l \in \mathbb{C P}^{2}$ consists of all points in $l$, where $l$ is now viewed as a complex line in $\mathbb{C}^{3}$. We can think of $S$ as being obtained from $\mathbb{C}^{3}$ by blowing up the origin, replacing it by a copy of $\mathbb{C} P^{2}$. The dual bundle $S^{*}$ is known as the hyperplane bundle. The Chern class of $\mathcal{Q}$ is easily computed to be $c=1+x+x^{2}$. In particular, the Euler number of the underlying real vector bundle is 1.

The reason for this detour on $\operatorname{spin}^{c}$ bundles is that every Aloff-Wallach space $N_{k, l}$ may be viewed as the boundary of some negative $\operatorname{spin}^{c}$ bundle $\mathbb{V}-(L)$, or rather a $\mathbb{Z}_{N}$

\footnotetext{
${ }^{11}$ In fact, this complex line bundle is precisely the universal subbundle $S$, defined below.
} 
quotient of such a bundle. However, in order to see this correspondence, we will have to make another slight digression.

Although the spin bundles $\Sigma_{ \pm}$do not in general exist globally on a four-manifold $B$, their projectivisations do exist as genuine bundles. In fact

$$
\mathbb{P} \Sigma_{ \pm}=\mathbb{P} \mathbb{V}_{ \pm}=\mathcal{S} \Lambda^{ \pm}
$$

independently of the choice of line bundle $L$. Recall that for any complex vector bundle $E$, one may define its projectivisation $\mathbb{P} E$ by replacing each complex fibre with the complex projective space one obtains by quotienting the complement of zero by the natural action of $\mathbb{C}^{*}$ (essentially, we quotient by the Hopf map on each fibre). The transition functions are simply those naturally induced by this construction.

In particular, if $E$ has complex rank two, then its projectivisation has fibre $\mathbb{C} \mathbf{P}^{1}=\mathbf{S}^{2}$. It follows that the boundary of $\mathbb{V}_{-}$, which is an $\mathbf{S}^{3}$ bundle over $B$, is the total space of a $U(1)$ bundle over the twistor space $\mathcal{S} \Lambda^{-}$of $B$. The associated complex line bundle is known as the hyperplane bundle ${ }^{\mathbb{1}} S^{*}$ of $\mathbb{V}_{-}$. So, the boundary of the spin ${ }^{c}$ bundle $\mathbb{V}_{-}$is the total space of a $U(1)$ bundle over the twistor space of $\mathbb{C P}^{2}$. This makes the connection between $\operatorname{spin}^{c}$ structures and Aloff-Wallach spaces - the latter may also be viewed as circle bundles over the twistor space, as we see from the rather useful diagram (4.12). Let us investigate this relation more carefully.

Complex line bundles over the twistor space are in 1-1 correspondence with elements of the second cohomology group $H^{2}\left(\mathcal{S} \Lambda^{-} ; \mathbb{Z}\right)$. For example, in the case of $B=\mathbb{C P}^{2}$, this group is isomorphic to $\mathbb{Z} \oplus \mathbb{Z}$. The two generators may be roughly thought of as the $\mathbb{C} \mathbf{P}^{1}$ that is linearly embedded in $\mathbb{C} \mathbf{P}^{2}$ (and generates $H_{2}\left(\mathbb{C P}^{2} ; \mathbb{Z}\right)$ ), and the $\mathbb{C} \mathbf{P}^{1}$ fibre. In general, if we denote $c_{1}\left(S^{*}\right)=y$ then $y \in H^{2}(\mathbb{P V} ; \mathbb{Z})$, and the restriction of $y$ to each $\mathbb{C P}^{1}=\mathbf{S}^{2}$ fibre of the twistor space generates the cohomology of the fibre. It follows from the Leray-Hirsch Theorem 43] that the cohomology ring of the twistor space $H^{*}\left(\mathcal{S} \Lambda^{-} ; \mathbb{Z}\right)$ is a free module over $H^{*}(B ; \mathbb{Z})$. Specifically,

$$
H^{*}\left(\mathcal{S} \Lambda^{-} ; \mathbb{Z}\right)=H^{*}\left(\mathbb{P} \mathbb{V}_{-} ; \mathbb{Z}\right)=H^{*}(B ; \mathbb{Z})[y] /\left(y^{2}+c_{1} y+c_{2}\right)
$$

where $c_{i}=c_{i}\left(\mathbb{V}_{-}\right)$are the Chern classes of the rank two complex vector bundle $\mathbb{V}_{-}$. This view of the Chern classes of a complex vector bundle was originally due to Grothendieck[13.

Now, the restriction of the unit sphere bundle in $S^{*}$ to any $\mathbf{S}^{2}$ fibre of the twistor space of course describes the Hopf map, $\mathcal{H}: \mathbf{S}^{3} \rightarrow \mathbf{S}^{2}$. Indeed, if $P: \mathcal{S} \Lambda^{-} \rightarrow B$ denotes

\footnotetext{
${ }^{12}$ This is not to be confused with the universal hyperplane bundle above, which is a complex line bundle over $\mathbb{C P}^{2}$.

${ }^{13}$ See 43] for an excellent summary.
} 
the projection map for the twistor space, viewed as an $\mathbf{S}^{2}$ bundle over $B$, then

$$
P_{*} y=1
$$

This equation in fact determines the cohomology class $y$ uniquely, up to the addition of the pull-back under $P$ of an element of $H^{2}(B ; \mathbb{Z})$. But this is just the choice of $\operatorname{spin}^{c}$ structure. To see this, note that tensoring $\mathbb{V}_{-}$with a line bundle $M$ with first Chern class $c_{1}(M)=a$ shifts

$$
\begin{array}{r}
c_{1} \rightarrow c_{1}+2 a \\
c_{2} \rightarrow c_{2}+c_{1} a+a^{2}
\end{array}
$$

which is the free transitive action of $H^{2}(B ; \mathbb{Z})$ on the space of $\operatorname{spin}^{c}$ structures. This is entirely equivalent to shifting the generator $y \rightarrow y+a$, in the representation (4.25), and is therefore equivalent to tensoring the hyperplane bundle $S^{*} \rightarrow S^{*} \otimes M$, as one would expect. The latter action is free and transitive on the space of circle bundles over the twistor space of $B$ satisfying the condition (4.26). Hence every such circle bundle (or equivalently complex line bundle) arises in this way.

We are now ready to make the connection with the Aloff-Wallach spaces. First, we consider a special case. Which $N_{k, l}$ spaces may be viewed as $\mathbf{S}^{3}$ bundles over $\mathbb{C P}^{2}$ ? We require $|k+l|=1$, which implies that $k= \pm 1-l$. Thus $N_{k, l}=N_{l,-k-l}=N_{l, \mp 1}=N_{\mp 1, l}$. Without loss of generality, we may take the plus sign, and hence we conclude that the only Aloff-Wallach spaces that may be viewed as three-sphere bundles over $\mathbb{C P}^{2}$ are, up to conjugacy, of the form $N_{1, p}$ for $p \in \mathbb{Z}$. Hence these spaces may be viewed as circle bundles over the twistor space satisfying (4.26), and therefore must be the boundary of some $\operatorname{spin}^{c}$ structure.

In fact, it is not hard to see that every $\operatorname{spin}^{c}$ structure arises in this way. Combining various formulae from above, it is easy to compute the Euler class of $\mathbb{V}_{-}(L)_{\mathbb{R}}$. Specifically, it is given by

$$
e\left(\mathbb{V}_{-}(L)_{\mathbb{R}}\right)=c_{2}\left(\mathbb{V}_{-}(L)\right)=x^{2}+\frac{1}{4}\left(c_{1}(L)-x\right)\left(c_{1}(L)+x\right)
$$

Hence the Euler number of the $\operatorname{spin}^{c}$ bundle of $\mathbb{C P}^{2}$ with line bundle $L$ is

$$
\chi\left(\mathbb{V}_{-}(L)_{\mathbb{R}}\right)=1+\frac{n^{2}-1}{4}
$$

where $c_{1}(L)=n x$ with $n$ an odd integer. Now examine the long exact cohomology sequence for the pair $(X, \partial X)$, where $X=\mathbb{V}_{-}(L)$ is the total space of some $\operatorname{spin}^{c}$ bundle. This reads 


$$
\ldots H^{4}(X, \partial X ; \mathbb{Z}) \stackrel{f}{\longrightarrow} H^{4}(X ; \mathbb{Z}) \stackrel{i^{*}}{\longrightarrow} H^{4}(\partial X ; \mathbb{Z}) \stackrel{\delta^{*}}{\longrightarrow} H^{5}(X, \partial X ; \mathbb{Z}) \longrightarrow \ldots
$$

where $i: \partial X \hookrightarrow X$ denotes inclusion, and $H^{4}(X, \partial X ; \mathbb{Z}) \cong H_{\text {cpct }}^{4}(X ; \mathbb{Z})$ is the same as the compactly supported cohomology. The map $f$ "forgets" that a cohomology class has compact support. By the isomorphism of Thom [44], we have

$$
H^{5}(X, \partial X ; \mathbb{Z}) \cong H^{1}\left(\mathbb{C} \mathbf{P}^{2} ; \mathbb{Z}\right) \cong 0
$$

since $X$ is the total space of a rank four bundle over $\mathbb{C P}^{2}$. Hence the exact cohomology sequence (4.30) implies that

$$
H^{4}(\partial X ; \mathbb{Z}) \cong H^{4}(X ; \mathbb{Z}) / f\left(H_{\text {cpct }}^{4}(X ; \mathbb{Z})\right)
$$

Now, the self-intersection number of $\mathbb{C} P^{2}$ inside $X$ is given by the Euler number $\chi=\left[\mathbb{C P}^{2}\right] \cdot\left[\mathbb{C P}^{2}\right][43]$. The lattice $H_{\text {cpct }}^{4}(X ; \mathbb{Z}) \cong H_{4}(X ; \mathbb{Z}) \cong \mathbb{Z}$ is generated by $\left[\mathbb{C P}^{2}\right]$, but the dual lattice $H^{4}(X ; \mathbb{Z})$ is generated by $\frac{1}{\chi}\left[\mathbb{C P}^{2}\right]$, since this has a scalar product of 1 with $\left[\mathbb{C P}^{2}\right]$. Putting these facts together, it follows that

$$
H^{4}\left(\partial \mathbb{V}_{-}(L) ; \mathbb{Z}\right) \cong\left(\frac{1}{\chi} \mathbb{Z}\right) / \mathbb{Z} \cong \mathbb{Z}_{\chi}
$$

Since the boundary of each $\operatorname{spin}^{c}$ bundle is supposed to be an Aloff-Wallach space of the form $N_{1, p}$, this is to be compared with the formula (4.17), which reads

$$
H^{4}\left(N_{1, p} ; \mathbb{Z}\right) \cong \mathbb{Z}_{r}
$$

where $r=\left|1+p+p^{2}\right|$. One easily sees that the formula relating $n$ (which determines the $\operatorname{spin}^{c}$ bundle) to $p$ (which determines the Aloff-Wallach space $N_{1, p}$ ) is

$$
n=2 p+1
$$

Indeed, substituting this expression into (4.29) gives

$$
\chi=1+\frac{n^{2}-1}{4}=1+\frac{(2 p+1)^{2}-1}{4}=1+p+p^{2}
$$

in agreement with (4.17). This shows the 1-1 correspondence explicitly. We note in passing that $N_{1,0}(n=1)$ corresponds to the boundary of the universal quotient bundle $\mathcal{Q}$ and $N_{1,1}(n=3)$ corresponds to the cotangent bundle $T^{*} \mathbb{C} \mathbf{P}^{2}$.

It is now simple to pass to the general case. Each $N_{k, l}$ space may be viewed as some $L(1, N)$ bundle over $\mathbb{C P}^{2}$ for some (various) $N$, given by (4.16). We may lift this 
bundle to its covering $\mathbf{S}^{3}$ bundle, which, as we have just seen, is the boundary of some $\operatorname{spin}^{c}$ bundle. Hence, in general, $N_{k, l}$ is the boundary of some $\mathbb{Z}_{N}$ quotient of a spin ${ }^{c}$ bundle. Note that projectivising this cyclic quotient, we find that the Chern class of the hyperplane bundle is now given by $N$ times the generator $y$.

We conclude this subsection with some remarks about Einstein metrics on AloffWallach spaces [42]. There exist two inequivalent Einstein metrics on each of the unexceptional $N_{k, l}$ spaces. These all have isometry group $S U(3) \times U(1)$ and weak $G 2$ holonomy. The latter fact means that the cones over these spaces are Spin(7) conifolds. The solutions found in [16] are resolutions of these conifolds. Of course, as we have explained in this section, typically these resolutions have orbifold singularities, and so strictly they are singular. The point is though that the orbifold singularity is much milder than the curvature singularity one encounters at the base of the conifolds. Indeed, the $A_{N-1}$ orbifold singularity will later be interpreted in Type IIA string theory as $N$ coincident D6-branes, so the singular nature of these solutions is actually rather desirable.

In contrast, $N_{1,-1}=N_{0,1}$ has one known Einstein metric, which also has weak $G 2$ holonomy and the same isometry group as the unexceptional cases. Again, there is a resolution of this cone [16]. This is not the same as the $\operatorname{Spin}(7)$ metric presented in the next section, which is asymptotically locally conical rather than asymptotically conical.

$N_{1,1}$ has two Einstein metrics, both with isometry groups $S U(3) \times S U(2)$, being respectively weak $G 2$ and tri-Sasakian. This means that the cones over these spaces are respectively $\operatorname{Spin}(7)$ and hyper-Kähler. Indeed, the resolutions of these cones are nothing but the metrics (2.6) and (2.7), respectively.

\subsection{Construction of New Spin(7) Metrics}

We turn now to the construction of the metrics. This generalises the work of [24]. We start by defining the generators of $S U(3)$, together with the associated left-invariant one-forms, which we denote $L_{A}{ }^{B}$, satisfying the exterior algebra

$$
d L_{A}{ }^{B}=i L_{A}{ }^{C} \wedge L_{C}{ }^{B}
$$

One must then split the generators into those that lie in the coset $S U(3) / U(1)$ and those that lie in the denominator $U(1)$. In particular, one must specify the $U(1)$ generator, $Q$. In the main text of [24], the choice of $U(1)$ generator corresponds to the Aloff-Wallach space $N_{1,1}$. The more general case was briefly mentioned in their Appendix C, although we shall use different notations. The generalisation to $N_{k, l}$ is 
obtained by setting

$$
Q \equiv \sqrt{\frac{2}{k^{2}+l^{2}}}\left(k L_{1}{ }^{1}+l L_{2}{ }^{2}\right)
$$

where the normalisation is chosen to coincide with Appendix $\mathrm{C}$ of [24]. There is one other $U(1)$ generator, $\lambda$, which lives in the coset space $S U(3) / U(1)$. This is orthogonal to $Q$, and together $Q$ and $\lambda$ generate the maximal torus of $S U(3)$. Specifically, we take

$$
\lambda \equiv \sqrt{\frac{2}{k^{2}+l^{2}}}\left(l L_{1}{ }^{1}-k L_{2}{ }^{2}\right)
$$

The remaining generators of $N_{k, l}$ are then

$$
\sigma \equiv L_{1}^{3}, \quad \Sigma \equiv L_{2}^{3}, \quad \nu \equiv L_{1}^{2}
$$

These are all complex, so one may split them into real and imaginary parts

$$
\sigma \equiv \sigma_{1}+i \sigma_{2}, \quad \Sigma \equiv \Sigma_{1}+i \Sigma_{2}, \quad \nu \equiv \nu_{1}+i \nu_{2}
$$

The exterior algebra (4.37) then reduces to

$$
\begin{aligned}
d \sigma_{1} & =-\alpha(2 l-k) \lambda \wedge \sigma_{2}-\alpha(2 k+l) Q \wedge \sigma_{2}-\nu_{1} \wedge \Sigma_{2}-\nu_{2} \wedge \Sigma_{1} \\
d \sigma_{2} & =\alpha(2 l-k) \lambda \wedge \sigma_{1}+\alpha(2 k+l) Q \wedge \sigma_{1}+\nu_{1} \wedge \Sigma_{1}-\nu_{2} \wedge \Sigma_{2} \\
d \Sigma_{1} & =-\alpha(l-2 k) \lambda \wedge \Sigma_{2}-\alpha(k+2 l) Q \wedge \Sigma_{2}-\nu_{1} \wedge \sigma_{2}+\nu_{2} \wedge \sigma_{1} \\
d \Sigma_{2} & =\alpha(l-2 k) \lambda \wedge \Sigma_{1}+\alpha(k+2 l) Q \wedge \Sigma_{1}+\nu_{1} \wedge \sigma_{1}+\nu_{2} \wedge \sigma_{2} \\
d \nu_{1} & =-\alpha(k+l) \lambda \wedge \nu_{2}-\alpha(k-l) Q \wedge \nu_{2}-\sigma_{2} \wedge \Sigma_{1}+\sigma_{1} \wedge \Sigma_{2} \\
d \nu_{2} & =\alpha(k+l) \lambda \wedge \nu_{1}+\alpha(k-l) Q \wedge \nu_{1}+\sigma_{1} \wedge \Sigma_{1}+\sigma_{2} \wedge \Sigma_{2} \\
d \lambda & =4 \alpha\left[(k+l) \nu_{1} \wedge \nu_{2}+l \sigma_{1} \wedge \sigma_{2}-k \Sigma_{1} \wedge \Sigma_{2}\right] \\
d Q & =4 \alpha\left[(k-l) \nu_{1} \wedge \nu_{2}+k \sigma_{1} \wedge \sigma_{2}+l \Sigma_{1} \wedge \Sigma_{2}\right]
\end{aligned}
$$

where the constant $\alpha=\alpha(k, l)=1 / \sqrt{2\left(k^{2}+l^{2}\right)}$.

It is easy to see how the Weyl group $\Sigma_{3}$ acts on these forms. For example, the element $w_{(2)} \in \Sigma_{3}$ defined by (4.5) permutes the labels "1" and "2" and hence induces

$$
\begin{aligned}
\nu & \mapsto \bar{\nu} \\
\sigma & \mapsto \Sigma \\
\Sigma & \mapsto \sigma
\end{aligned}
$$


On the other hand, the order three element $w_{(3)}$ defined by 4.7 cyclically permutes (up to complex conjugation) the three one-forms:

$$
\begin{aligned}
\nu & \mapsto \bar{\sigma} \\
\sigma & \mapsto \bar{\Sigma} \\
\Sigma & \mapsto \nu
\end{aligned}
$$

One may check that performing this transformation twice more brings one back to the initial ordering. On the other hand, the $\mathbb{Z}_{2}$ group of outer automorphisms of $S U(3)$ acts naturally by complex conjugation: $\Sigma \rightarrow \bar{\Sigma}$, etc.

The metric ansatz we take is the cohomogeneity one ansatz

$$
d s^{2}=d t^{2}+f^{2} \lambda^{2}+a^{2}\left(\sigma_{1}^{2}+\sigma_{2}^{2}\right)+b^{2}\left(\Sigma_{1}^{2}+\Sigma_{2}^{2}\right)+c^{2}\left(\nu_{1}^{2}+\nu_{2}^{2}\right)
$$

where $a, b, c$ and $f$ are functions of the "radial" variable $t$. The following system of first-order equations describe a solution of $\operatorname{Spin}(7)$ holonomy

$$
\begin{aligned}
\frac{\dot{a}}{a} & =\frac{b^{2}+c^{2}-a^{2}}{a b c}-\frac{2 \alpha l f}{a^{2}} \\
\frac{\dot{b}}{b} & =\frac{a^{2}+c^{2}-b^{2}}{a b c}-\frac{2 \alpha k f}{b^{2}} \\
\frac{\dot{c}}{c} & =\frac{a^{2}+b^{2}-c^{2}}{a b c}+\frac{2 \alpha(k+l) f}{c^{2}} \\
\frac{\dot{f}}{f} & =-\frac{2 \alpha f(k+l)}{c^{2}}+\frac{2 \alpha l f}{a^{2}}+\frac{2 \alpha k f}{b^{2}}
\end{aligned}
$$

where $\dot{a}=\frac{d a}{d t}$, etc. To see this, we introduce the orthonormal basis

$$
\begin{aligned}
& e^{1}=\frac{d r}{f}, \quad e^{2}=f \lambda, \quad e^{3}=a \sigma_{1}, \quad e^{4}=a \sigma_{2}, \\
& e^{5}=c \nu_{1}, \quad e^{6}=c \nu_{2}, \quad e^{7}=b \Sigma_{1}, \quad e^{8}=b \Sigma_{2}
\end{aligned}
$$

The metric (4.45) now becomes

$$
d s^{2}=\sum_{i=1}^{8} e^{i} \otimes e^{i}
$$

Denoting $e^{i j k l}=e^{i} \wedge e^{j} \wedge e^{k} \wedge e^{l}$, we find that 


$$
\begin{aligned}
\Phi= & -e^{1234}+e^{1256}+e^{1278}-e^{1367}-e^{1358}-e^{1468}+e^{1457} \\
& +e^{2368}-e^{2357}-e^{2467}-e^{2458}-e^{3456}-e^{3478}+e^{5678}
\end{aligned}
$$

is self-dual, and imposing $d \Phi=0$ (which is equivalent to $\Phi$ being harmonic) precisely reproduces the first-order system (4.46). The self-dual harmonic four-form $\Phi$ is precisely the Cayley form that determines the reduction of the structure group from $\operatorname{Spin}(8)$ to $\operatorname{Spin}(7)$.

Notice that setting $f \equiv 0$ and $a \equiv b$ leads to the following consistent truncation

$$
\begin{aligned}
\dot{a} & =\frac{c}{a} \\
\dot{c} & =2-\frac{c^{2}}{a^{2}}
\end{aligned}
$$

independently of $k$ and $l$. These equations describe the known metric of $G 2$ holonomy on the bundle of anti-self-dual two-forms over $\mathbb{C P}^{2}$, (2.3). This is hardly surprising. Setting $f=0$ roughly corresponds to removing the twisting due to the D6-branes (this will be explained in the next section), and $a=b$ yields the standard Fubini-Study metric on the $\mathbb{C P}^{2}$ base space.

\subsection{New $\operatorname{Spin}(7)$ Metrics on $\mathcal{Q}$}

Now consider setting $k=0, l=1$. Defining the new radial coordinate $d r=F d t$, where $F=\sqrt{2} f$, the general system (4.46) becomes

$$
\begin{aligned}
a^{\prime} & =\frac{b^{2}+c^{2}-a^{2}}{b c F}-\frac{1}{a} \\
b^{\prime} & =\frac{a^{2}+c^{2}-b^{2}}{a c F} \\
c^{\prime} & =\frac{a^{2}+b^{2}-c^{2}}{a b F}+\frac{1}{c} \\
F^{\prime} & =\frac{F}{a^{2}}-\frac{F}{c^{2}}
\end{aligned}
$$

where a prime denotes derivative with respect to $r$. These equations are remarkably similar to the first-order equations describing $G 2$ metrics on the spin bundle of $\mathbf{S}^{3}$ found in 45 . In fact, the system possesses a similar $\mathbb{Z}_{2}$ symmetry 


$$
r \rightarrow-r, \quad a \leftrightarrow c, \quad b \rightarrow-b
$$

We have been able to find the following explicit solution

$$
\begin{aligned}
a^{2} & =\left(r+\frac{2}{3}\right)\left(r-\frac{4}{3}\right) \\
b^{2} & =r^{2} \\
c^{2} & =\left(r-\frac{2}{3}\right)\left(r+\frac{4}{3}\right) \\
F^{2} & =\frac{\left(r-\frac{4}{3}\right)\left(r+\frac{4}{3}\right)}{\left(r-\frac{2}{3}\right)\left(r+\frac{2}{3}\right)}
\end{aligned}
$$

This in fact gives us a one-parameter family of solutions. To see this, note that rescaling the metric $g \rightarrow \frac{9 a^{2}}{4} g$ and then scaling $r \rightarrow 2 r / 3 a$ also gives us a metric of $\operatorname{Spin}(7)$ holonomy, given explicitly by

$$
\begin{aligned}
d s^{2}= & \frac{(r-a)(r+a)}{(r-2 a)(r+2 a)} d r^{2}+\frac{9 a^{2}}{8} \frac{(r-2 a)(r+2 a)}{(r-a)(r+a)} \lambda^{2}+(r+a)(r-2 a)\left(\sigma_{1}^{2}+\sigma_{2}^{2}\right)+ \\
& +r^{2}\left(\Sigma_{1}^{2}+\Sigma_{2}^{2}\right)+(r-a)(r+2 a)\left(\nu_{1}^{2}+\nu_{2}^{2}\right)
\end{aligned}
$$

This is a complete metric on the universal quotient bundle $\mathcal{Q}$ of $\mathbb{C} \mathbf{P}^{2}$, for either $r>2 a$ or $r<-2 a$. As in [45], the two solutions are interchanged by the $\mathbb{Z}_{2}$ symmetry. We choose to take $r>2 a$.

To see that this is a smooth complete metric on $\mathcal{Q}$, set $\rho^{2}=3 a(r-2 a)$. Then, near $\rho=0$, the metric approaches

$$
d s^{2}=\left[d \rho^{2}+\rho^{2}\left(\frac{1}{2} \lambda^{2}+\sigma_{1}^{2}+\sigma_{2}^{2}\right)\right]+4 a^{2}\left(\nu_{1}^{2}+\nu_{2}^{2}+\Sigma_{1}^{2}+\Sigma_{2}^{2}\right)
$$

The metric in square brackets is in fact the standard Euclidean metric on $\mathbb{R}^{4}$. This may be seen by rescaling the variables

$$
\begin{gathered}
\eta_{1} \equiv 2 \sigma_{1} \\
\eta_{2} \equiv 2 \sigma_{2} \\
\eta_{3} \equiv \sqrt{2} \lambda
\end{gathered}
$$

The three one-forms $\eta_{i}$ may now be written as $\eta_{i}=s_{i}+\ldots$ where the $s_{i}$ are a set of left-invariant one-forms on the $S U(2) \cong \mathbf{S}^{3}$ fibres, and "..." denotes additional terms 
that describe the twisting of the fibres over the base $\mathbb{C} P^{2}$. These latter terms show up in the exterior algebra (4.42). The metric in square brackets is now

$$
d \rho^{2}+\frac{\rho^{2}}{4}\left(\eta_{1}^{2}+\eta_{2}^{2}+\eta_{3}^{2}\right)
$$

which is clearly the usual metric on $\mathbb{R}^{4}$ in spherical polars. The induced metric on $\rho=0$ is the standard Fubini-Study metric on $\mathbb{C P}^{2}$, up to a scale factor. Thus the principal $N_{0,1}=S U(3) / U(1)$ orbits collapse smoothly down to a $\mathbb{C P}^{2}$ bolt. One must be slightly cautious when interpreting the global structure of (4.57) - the periodicity of $\lambda$ may be such that we obtain an orbifold $\mathbb{R}^{4} / \mathbb{Z}_{N}$, with $N>1$, rather than $\mathbb{R}^{4}$. However, since the level surfaces are $N_{0,1}$, we know from our discussion of Aloff-Wallach spaces that $N_{0,1}=N_{1,0}$ may indeed be viewed as the boundary of an $\mathbb{R}^{4}$ bundle over $\mathbb{C P}^{2}$ specifically, a $\operatorname{spin}^{c}$ structure. As we demostrated in a previous section, this bundle is precisely the universal quotient bundle $\mathcal{Q}$.

At large distances $(r \rightarrow \infty)$, the metric asymptotes to the geometry

$$
d s^{2}=\frac{9 a^{2}}{8} \lambda^{2}+d r^{2}+r^{2}\left(\sigma_{1}^{2}+\sigma_{2}^{2}+\nu_{1}^{2}+\nu_{2}^{2}+\Sigma_{1}^{2}+\Sigma_{2}^{2}\right)
$$

Thus the $U(1)$ fibres tend to a constant length while the other directions expand linearly with radius. This behaviour is somewhat similar to that of the Taub-NUT metric, which of course describes a flat D6-brane in flat space. In the case at hand, the metric asymptotes to $\mathbf{S}^{1} \times C\left(\mathcal{S} \Lambda^{-}\right)$where $C\left(\mathcal{S} \Lambda^{-}\right)$is the cone over the $\mathbb{C P}^{2}$ twistor space $\mathcal{S} \Lambda^{-}=S U(3) / T^{2}$. However, as for the $G 2$ metric on $\Lambda^{-}$, the twistor space metric asymptotes to the squashed Einstein metric, rather than the usual one, [11]. Our metric is therefore asymptotically locally conical, or ALC. Notice that the functional form of the metric (4.54) is extremely similar to that of the ALC Spin(7) metric on $\Sigma_{-} \mathbf{S}^{4}(2.5)$.

\section{Flux Quantisation in M-Theory on Spin(7) Man- ifolds}

In this section we study in detail various M-theoretic aspects of our models. We find in particular that the M-theory lift of a Type IIA configuration of D6-branes wrapping a coassociative cycle is always described by the total space of a $\operatorname{spin}^{c}$ bundle. We study also the quantisation of the M-theory four-form $G$ on the various $\operatorname{Spin}(7)$ manifolds of interest. We find that in all cases one must turn on a half-integral $G$-flux in order that the M-theory solutions be consistent. 


\subsection{D6-branes Wrapping Coassociative Cycles}

We begin this subsection by describing the lift to M-theory of a configuration of D6branes wrapping a coassociative cycle of a $G 2$-manifold $M^{7}$. The mathematical description of this lift ties in very closely with our previous discussion of circle bundles over twistor spaces, and also with the work of McLean on the deformability of supersymmetric cycles [22].

Suppose then that the Type IIA manifold takes the form of a metric product $\mathbb{R}^{3} \times$ $M^{7}$ of Minkowski three-space with $M^{7}$, and consider wrapping $N$ D6-branes over the submanifold $W=\mathbb{R}^{3} \times B$. As usual, $M^{7}$ is one of the non-compact $G 2$ manifolds of section 2 , and $B$ is a coassociative submanifold of $M^{7}$. By supersymmetry, the lift of this configuration to M-theory should be described locally by a manifold (or, more precisely, an orbifold) of $\operatorname{Spin}(7)$ holonomy [12].

From [22] we know that $N B$ must be the bundle of anti-self-dual two-forms $\Lambda^{-} B$ over $B$. Indeed, the explicitly known $G 2$ metrics presented in section (2.2) are defined on the total space of such a bundle. Since we are primarily interested in the local physics near the D6-branes, we may therefore restrict our attention to this case. Notice that $\Lambda^{-} B$ is $\operatorname{spin}^{c}$ since any oriented vector bundle over a four-manifold $B$ is $\operatorname{spin}^{c}$ 46]. This fact is important since $W$ must be $\operatorname{spin}^{c}$ in order that wrapping branes on $W$ makes sense. Otherwise, one encounters global anomalies on the string worldsheet [38], and there is no K-theoretic interpretation of D-brane charge [47].

The D6-branes are viewed, at the IIA level, as probe branes. That is, one ignores the gravitational back-reaction of the branes on the geometry. The presence of the branes implies that the RR two-form $G_{2}$ has a delta-function singularity on $W$

$$
\left[d G_{2}\right]=N \delta(W)
$$

where $\delta(W)$ is Poincaré dual to the worldvolume $W$, and has support on $W$. Equation (5.1) is the statement that the D6-branes act as a magnetic source for $G_{2}$. However, on the complement of $W, G_{2}$ is closed. We may then interpret $\left[G_{2} / 2 \pi\right]$ as the first Chern class of the "M-theory circle bundle" over the complement of $W$. Thus $\left[\frac{G_{2}}{2 \pi}\right]=c_{1}(\mathcal{L})$, where $\mathcal{L}$ is a complex line bundle over $\mathbb{R}^{3} \times M^{7} \backslash W \equiv M_{0}^{10}$.

The total M-theory space $M^{11}$ is a degenerate circle bundle over the Type IIA manifold $M^{10}=\mathbb{R}^{3} \times M^{7}$, with the circle fibres collapsing to zero on a copy of the D6-brane worldvolume $W$. More precisely, the complement of this "lift" of $W$ is the total space of the bundle of unit vectors $\mathcal{S} \mathcal{L}$ in $\mathcal{L}$, which in a tubular neighbourhood of $W$ is also an $L(1, N)$ bundle over $W$. This Lens space bundle is then "filled in" with the associated $\mathbb{R}^{4} / \mathbb{Z}_{N}$ bundle, whose zero section is the copy of $W$. On occasion, it

will be important to distinguish logically between the brane worldvolume $W \subset M^{10}$ 
in Type IIA and its lift $W \subset M^{11}$ in M-theory. For example, in a later subsection we will refer to the latter as $\widehat{W}$. Hopefully the context should make it clear as to which submanifold we are referring.

Now, going back to Type IIA, the RR two-form $G_{2}$ satisfies

$$
P_{*}\left(\frac{G_{2}}{2 \pi}\right)=N
$$

where $P: \mathcal{S} N W \rightarrow W$ denotes the $\epsilon$-sphere bundle of the normal bundle of $W$ in $\mathbb{R}^{3} \times M^{7}$. This may be thought of as consisting of all points which are at a distance $\epsilon>0$ from $W$. Equation (5.2) determines $G_{2}$ up to a shift $G_{2} \rightarrow G_{2}+2 \pi P^{*}(a)$ where $a \in H^{2}(W ; \mathbb{Z}) \cong H^{2}(B ; \mathbb{Z})$. Shifting by different values of $a$ results in a different lift to M-theory, since $\left[G_{2} / 2 \pi\right]$ is the first Chern class of the M-theory circle bundle $\mathcal{S} \mathcal{L}$. Thus in order to describe the lift of the D6-brane configuration to M-theory, one must specify the flux of $G_{2}$ over $B$.

Now, the twistor space $\mathcal{S} \Lambda^{-} B$ may be described in terms of the projectivisation of the negative $\operatorname{spin}^{c}$ bundles

$$
\mathcal{S} \Lambda^{-} \cong \mathbb{P} \mathbb{V}_{-}(L) \cong \mathbb{P}\left(\Sigma_{-} \otimes L^{1 / 2}\right)
$$

The ambiguity in the choice of $G_{2}$ flux over $B$ is precisely the ambiguity in the choice of $\operatorname{spin}^{c}$ structure, just as in section 4 . Hence we conclude that the lift to Mtheory of a configuration of D6-branes wrapped over a coassociative submanifold $B$ is always described locally by a $\operatorname{spin}^{c}$ bundle over $B$, and, moveover, this correspondence is actually 1-1. This gives the fact that we have a choice of $\operatorname{spin}^{c}$ bundle for our $\operatorname{Spin}(7)$ manifold, which in turn describes the M-theory manifold, a more physical meaning it is just the choice of $G_{2}$ flux over $B$. For the case $B=\mathbb{C P}^{2}$, we also know that this choice of $G_{2}$ flux corresponds to a choice of Aloff-Wallach space that bounds the total space of the appropriate $\operatorname{spin}^{c}$ bundle.

The case of $B=\mathbf{S}^{4}$ is more straightforward. Since $H^{2}\left(\mathbf{S}^{4} ; \mathbb{Z}\right)=0$, there is no flux of $G_{2}$ over $\mathbf{S}^{4}$, and therefore the lift to M-theory is entirely determined by the number of D6-branes. From our above remarks, the lift of a single D6-brane wrapped on the coassociative $\mathbf{S}^{4}$ of $\Lambda^{-} \mathbf{S}^{4}$ is a $\operatorname{Spin}(7)$ metric on the bundle of negative chirality spinors $\Sigma_{-}$over $\mathbf{S}^{4}$. The choice of $\operatorname{spin}^{c}$ structure is unique in this case since the line bundle $L$ must be trivial. Specfically, the $\operatorname{spin}^{c}$ bundle is just the spin bundle. The appropriate $\operatorname{Spin}(7)$ metric is of course (2.5). These facts fit in nicely with the work of McLean [22], where it was shown that the normal bundle of a Cayley $\mathbf{S}^{4}$ is precisely the bundle

\footnotetext{
${ }^{14}$ For $W$ non-compact, one would generally need to take $\epsilon$ to be a positive function on $W$ in order that the image of $\mathcal{S} N W$ under the exponential map is an embedding.
} 
$\Sigma_{-}$. Hence the results of [22] are consistent with the lift to M-theory described in this section. This lift was also constructed explicitly in the context of gauged supergravity in [48], where the AC Spin(7) metric (2.4) was obtained by lifting to M-theory a $D=8$ supergravity solution describing D6-branes wrapped on the coassociative $\mathbf{S}^{4}$ of $\Lambda^{-} \mathbf{S}^{4}$. Of course, for $N>1$ branes, one simply takes the $\mathbb{Z}_{N}$ quotient of the spin bundle $\Sigma_{-}$.

Notice that this reasoning actually implies the existence of the $\operatorname{Spin}(7)$ spaces found recently in [16], and gives them a physical interpretation within string theory.

\subsection{Flux Quantisation}

At the classical level, that is all there is to say. The M-theory manifold satisfies the classical (eleven-dimensional supergravity) field equations with $G=0$, and preserves a certain fraction (namely $\frac{1}{16}$ ) of the (maximal) supersymmetry of the vacuum. However, as we saw in the last section, in the quantum theory things are more subtle. In particular, if $w_{4}(M)$ is not zero, the periods of $G / 2 \pi$ must be half-integral in order that the membrane path integral make sense. It follows that if $\lambda$ is not divisible by two, one must turn on a half quantum of $G$-flux in order that the M-theory solution be consistent. We shall find that this is the case for our examples.

Consider first the case $X=\Sigma_{-} \mathbf{S}^{4}$. Since $p_{1}\left(T \mathbf{S}^{4}\right)=0$ and $p_{1}\left(\Sigma_{-} \mathbf{S}^{4}\right)=-2 u$, we have $\left.p_{1}(T X)\right|_{\mathbf{S}^{4}}=-2 u$, where $u$ is a generator of $H^{4}\left(\mathbf{S}^{4} ; \mathbb{Z}\right) \cong \mathbb{Z}$. Hence the restriction of $\lambda(T X)=p_{1}(T X) / 2$ to $\mathbf{S}^{4}$ generates the cohomology of $\mathbf{S}^{4}$. Notice that this is also the Euler class of the bundle $\Sigma_{-} \mathbf{S}^{4}$. In particular though, $\lambda$ is not divisible by two.

We turn to $X=\mathbb{V}_{-}(L)$ with $L$ an "odd" complex line bundle over $\mathbb{C P}^{2}$ - that is, $c_{1}(L)$ is an odd integer in $H^{2}\left(\mathbb{C P}^{2} ; \mathbb{Z}\right) \cong \mathbb{Z}$. In this case, $p_{1}\left(T \mathbb{C} \mathbf{P}^{2}\right)=3 x^{2}$ and $p_{1}\left(\mathbb{V}_{-}(L)_{\mathbb{R}}\right)=-x^{2}+\frac{1}{2}\left(c_{1}(L)-x\right)\left(c_{1}(L)+x\right)$, where $x$ generates $H^{2}\left(\mathbb{C P}^{2} ; \mathbb{Z}\right)$. Hence

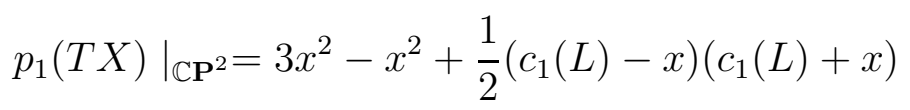

and so

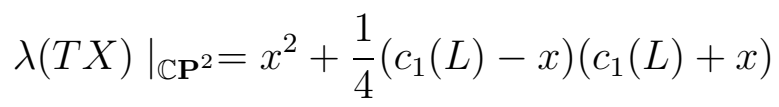

Of course, $\left(c_{1}(L)-x\right)\left(c_{1}(L)+x\right)$ is always divisible by eight, so that $\lambda$ is an integral class. But we also see from this that $\lambda$ is not divisible by two. Indeed, on $\mathbb{C P}^{2} \lambda(T X)$ restricts to the Euler class of $\mathbb{V}_{-}(L)_{\mathbb{R}}$, which mod two generates $H^{4}\left(\mathbb{C P}^{2} ; \mathbb{Z}_{2}\right)$.

\footnotetext{
${ }^{15}$ Theorem 20.8 of [44] states that, up to two-torsion (which is irrelevant here), $p(T E)=$ $\pi^{*}(p(E) p(T B))$ where $\pi: E \rightarrow B$ is a smooth vector bundle over $B$.
} 
Hence the fourth Stiefel-Whitney class of the tangent bundle of $X$, restricted to $B$, generates $H^{4}\left(B ; \mathbb{Z}_{2}\right)$, in all cases. In particular, it cannot be zero, and hence $\lambda(X)$ is not divisible by two. Thus M-theory on either the total space of $\Sigma_{-} \mathbf{S}^{4}$, or any $\operatorname{spin}^{c}$ bundle $\mathbb{V}_{-}$over $\mathbb{C P}^{2}$ is consistent only if one turns on a half-integral $G$-flux. But our D6-brane configuration contained no such $G$-flux! We can only conclude that the D6brane configuration we started with must have been inconsistent in some way. Indeed, this is the correct conclusion. One is forced to turn on half-integral $G_{4}$ in Type IIA.

The meaning of this non-zero $G_{4}$ is at first slightly mysterious. For M-theory on the product $M^{11}=S^{1} \times M^{10}$, non-zero $w_{4}\left(M^{11}\right)$ is equivalent to non-zero $w_{4}\left(M^{10}\right)$. Indeed, the shift in quantisation of $G$ in [18] was derived by analysing global anomalies on the M2-brane worldvolume. The analysis for $G_{4}$ goes through in precisely the same way, with D2-branes in Type IIA string theory, rather than M2-branes in M-theory. It is easy to check ${ }^{\mathbb{D}}$ that $w_{4}\left(T M^{7}\right)=0$ with $M^{7}$ either of the total spaces of the bundle of anti-self-dual two-forms over $\mathbf{S}^{4}$ or $\mathbb{C P}^{2}$. So if we were to consider M-theory on either of these spaces, we would find no shift in the quantisation law for $G$. Hence in the recent studies of M-theory on G2 manifolds [2] no such shift in the $G$-flux would have been found. In fact, $w_{4}\left(M^{7}\right)=0$ is always zero for a compact spin seven-manifold $M^{7}$. To see this, note that one can write the fourth Stiefel-Whitney class as

$$
w_{4}\left(M^{7}\right)=\sum_{i+j=4} \operatorname{Sq}^{i}\left(V_{j}\right)
$$

where $\mathrm{Sq}^{i}$ denotes a certain mod 2 cohomology operation known as the $i^{\text {th }}$ Steenrod square, and $V_{j} \in H^{j}\left(M^{7} ; \mathbb{Z}_{2}\right)$ denotes the $j^{\text {th }} \mathrm{Wu}$ class of $M^{7}$ [4]. Now, $V_{4}=0$ on dimensional grounds, and the Steenrod squares annihilate the $V_{1}$ and $V_{2}$ terms for a similar reason. Hence we are left with

$$
w_{4}\left(M^{7}\right)=\mathrm{Sq}^{1}\left(V_{3}\right)+\mathrm{Sq}^{2}\left(V_{2}\right)
$$

Now, $V_{2}=w_{1}^{2}\left(M^{7}\right)+w_{2}\left(M^{7}\right)$, and so this is zero as $M^{7}$ is spin. Similarly, $V_{3}$ may be written as a polynomial in Stiefel-Whitney classes of $M^{7}$, and so $V_{3}$ also vanishes for the same reason. Hence we conclude that $w_{4}\left(M^{7}\right)=0$. Note that this argument breaks down for spin eight-manifolds, as $V_{4}$ is no longer zero in general.

So, where does this half integral flux come from? The key is that the presence of the D6-branes implies that the M-theory manifold is not just a product of the Type IIA manifold with a circle. In fact, this contribution to $G_{4}$ is K-theoretic in nature, as we shall now explain.

\footnotetext{
${ }^{16}$ The reader may verify this using the Theorem quoted in the last footnote, together with the results derived in Appendix A.
} 


\subsection{G-Flux from K-Theory}

In this subsection we will find that the shift in quantisation law for $G$ found in the last subsection is related to the K-theory classification of RR-fields in Type IIA string theory. A detailed knowledge of K-theory will not be required in order to follow the argument, provided one is willing to accept certain facts taken from the literature without proof.

In the absence of branes, RR fields in Type IIA string theory on $\square M_{0}^{10}$ are, roughly speaking, classified by an element of the K-group $K\left(M_{0}^{10}\right)$. Specifically, given an element $v \in K\left(M_{0}^{10}\right)$, one has

$$
\frac{G_{*}}{2 \pi}=\sqrt{\hat{A}} \operatorname{Ch}(v+\theta / 2)
$$

where $G_{*}=G_{0}+G_{2}+G_{4}+\ldots$ is the total RR-form field strength, $\hat{A}$ is the usual Dirac genus, which will not enter our discussion here, and

$$
\text { Ch }: K\left(M_{0}^{10}\right) \rightarrow H^{\text {even }}\left(M_{0}^{10} ; \mathbb{Q}\right)
$$

is the Chern character, which maps an element of the K-group to an even cohomology class. The $\theta$ characteristic lives in the space $\Gamma_{1} / 2 \Gamma_{1}$ where the lattice $\Gamma=K\left(M_{0}^{10}\right) / K\left(M_{0}^{10}\right)_{\text {tors }}$ splits as a direct sum $\Gamma_{1} \oplus \Gamma_{2}$. The defining property of the sublattice $\Gamma_{1}$ is that it is maximally Langrangian; that is, it is a maximal sublattice such that $\omega(x, y)=0, \forall x, y \in \Gamma_{1}$ where $\omega$ is a certain natural symplectic form on $\Gamma$. The interested reader is referred to [49] and references therein for more details.

Fortunately, we do not need to know much about $\theta$ in the present paper. We extract the following formulae from [49]

$$
G_{0}(\theta)=G_{2}(\theta)=0
$$

and

$$
\frac{G_{4}(\theta)}{2 \pi}=-\lambda \quad(\bmod 2)
$$

The mod two is inserted to remove from $G_{4}(\theta)$ various terms that won't interest us in the present discussion. Note that it is the contribution of the $\theta$ characteristic that gives the shift in $G_{4}$ analagous to the shift of $G$ in M-theory. Since $\lambda=0$ modulo two for spin seven-manifolds, we may ignore the $\theta$ characteristic from here on.

\footnotetext{
${ }^{17}$ The notation $M_{0}^{10}$ may seem a little cumbersome, but the space to which we shall eventually apply these facts will be denoted in this way. It is merely the complement of the D6-brane worldvolume $W$.
} 
With this proviso, and assuming also that the class $v$ satisfies $G_{0}=0$, we have

$$
\begin{gathered}
{\left[G_{2} / 2 \pi\right]=c_{1}(v)} \\
{\left[G_{4} / 2 \pi\right]=\frac{1}{2} c_{1}(v)^{2}-c_{2}(v)}
\end{gathered}
$$

where the last formula receives corrections from $\theta$ that will not interest us (as they are integral). We interpret $\left[G_{2} / 2 \pi\right]=c_{1}(v)=c_{1}(\mathcal{L})$ in terms of the first Chern class of a complex line bundle $\mathcal{L}$, and $-c_{2}(v)$ is identified with the characteristic class of an $E_{8}$ bundle over $M_{0}^{10}$. This class specifies the $E_{8}$ bundle uniquely, up to bundle isomorphism [40]. Again, we shall not make use of this fact.

Now, $\left[G_{2} / 2 \pi\right]$ is an integral class, as it is a Chern class. Similarly, $c_{2}(v)$ is integral. However, the fact that RR fields live in K-theory and not cohomology means that, when one projects from K-theory to cohomology via the Chern character, the fields become mixed. In particular, as we see from equation (5.13), $G_{4}$ depends on $G_{2}$. If $c_{1}(v)^{2}$ is not divisible by two, then (5.13) implies that $\left[G_{4} / 2 \pi\right]$ is half-integral, and, in particular, one cannot set it to zero.

These comments apply to IIA string theory in the absence of branes. Consider our general setup, with $N$ D6-branes wrapped over some oriented $\operatorname{spin}^{c}$ submanifold $W$ of $M^{10}$, which is oriented and spin. Note also that $M^{10}$ spin implies that $w_{2}(T W)=$ $w_{2}(N W)$. A single D6-brane (corresponding to $N=1$ ) lifts to the manifold $M^{11}$ in M-theory, with $W$ lifting to $\widehat{W}$, and $N W_{0}$ lifting to $N \widehat{W}_{0}$ (see the discussion in section 5.1. A subscript zero always denotes the complement of (the) zero (section)). The case $N>1$ is obtained by taking the obvious $\mathbb{Z}_{N}$ quotient. The presence of the D6-branes implies that $G_{2}$ has a delta-function singularity on $W$ (5.1), but is otherwise closed on the complement of $W$ in $M_{10}$.

Consider now excising the brane worldvolume $W$ from $M^{10}$ to yield the space $M_{0}^{10}$ (for technical purposes, one may actually want to remove a small tubular neighbourhood of $W$ from $M^{10}$ to form $M_{0}^{10}$, and later take a limit). $G_{2}$ is closed on $M_{0}^{10}$ and there are no longer any branes. However, the fact that there was a brane where $W$ used to be is encoded by the equation

$$
P_{*}\left(\frac{G_{2}}{2 \pi}\right)=N
$$

This is in fact the same statement as (5.2).

We are left with RR-flux on $M_{0}^{10}$ without any brane sources. Our main technical assumption is that this configuration corresponds to some K-theory class $v \in K\left(M_{0}^{10}\right)$. 
This is in spirit with the discussion of the K-theory classification of RR-fields in [50]. Indeed, the presence of a brane on $M^{10}$ induces a RR-field $K\left(\partial M^{10}\right)$ on the "boundary at infinity", $\partial M^{10}$. In the present situation, $\partial M^{10}$ is a deformation retraction of $M_{0}^{10}$. K-groups are homotopy invariant, and so one may equivalently consider the brane as inducing a K-Theory class in $K\left(M_{0}^{10}\right)$.

With $G_{0}=0$ (this corresponds to no D8-brane flux, which seems natural as we started with D6-branes. Also, there is no M-theory interpretation of $G_{0}$, so we cannot lift this to compare with our analagous discussion in M-theory) it follows that

$$
\left[\frac{G_{2}}{2 \pi}\right]=c_{1}(v)=c_{1}(\mathcal{L})
$$

and there is a possible half-integral contribution to $\left[G_{4} / 2 \pi\right]$ of the form $\left[\frac{1}{2} c_{1}(\mathcal{L})^{2}\right.$. It is precisely this contribution to $G_{4}$ that we now focus on, ignoring any other integral part.

We would like to lift the present situation to M-theory. $G_{4}$ lifts directly to $G$, the M-theory four-form. We have already seen in the last subsection that in some cases $[G / 2 \pi]$ must be half-integral in the quantum theory. Following [49, we must shift $G / 2 \pi$ by a representative of $\frac{1}{2} c_{1}(\mathcal{L})^{2}$. This class, although non-trivial in $M_{0}^{10}$ is trivialised tautologically when pulled back to $M_{0}^{11}$, the total space of $S \mathcal{L}$. To see this, one introduces the one-form $\omega=(d \tau+A) / 2 \pi$ where $\tau$ parametrises the M-theory circle direction, and $A$ is a connection on $\mathcal{L}$. $\omega$ integrates to 1 on each $\mathbf{S}^{1}$ fibre, and satisfies $d \omega=F / 2 \pi$ where $F$ is the curvature of $\mathcal{L}$. Indeed, the $\omega$ of 49 is in fact a global angular form on $S \mathcal{L}$, and its exterior derivative is therefore topologically trivial. The point is that a gauge transformation $\tau \rightarrow \tau+\phi$ is cancelled by the corresponding gauge transformation of the connection, $\mathcal{A} \rightarrow \mathcal{A}-d \phi$, and hence $\omega$ is globally defined, even though its constituents are not.

One now shifts $C$ by $\delta C=\pi \omega \wedge d \omega$, and correspondingly $G / 2 \pi=d C / 2 \pi$ is shifted by $\delta G / 2 \pi=\frac{1}{2} F \wedge F /(2 \pi)^{2}$. Thus $G / 2 \pi$ has been shifted by the pull-back to $M_{0}^{11}$ of $\frac{1}{2} c_{1}(\mathcal{L})^{2}$, which is topologically trivial.

Now, our shift in $G$ was only defined on $M_{0}^{11}$, not on $M^{11}$. Recall that the former is obtained from the latter by excising $\widehat{W}$. However, the fact that the shift in cohomology class is trivial on $M_{0}^{11}$ does not imply that the shift is trivial when extended to $M^{11}$. This is just as well, otherwise we could not explain the half-integral of flux we found in M-theory using K-theory!

To see this, consider the shift in the $C$ field, $\delta C=\pi \omega \wedge d \omega$. This is a globally defined

\footnotetext{
${ }^{18}$ Recall that $\mathcal{L}$ is a complex line bundle over $M_{0}^{10}$, whose bundle of unit vectors coincides with the "M-theory circle direction".
} 
three-form on $N \widehat{W}_{0}$, where recall that $\widehat{W}$ is the lift of the D6-brane worldvolume $W$ to $M^{11}$. Consider integrating this form over a Lens space $L(1, N)$ sitting in some normal space to $\widehat{W}$ in $M^{11}$. The one-form $\omega$ integrates to 1 on the circle direction, and $d \omega=F / 2 \pi$ integrates to $N$ on the two-sphere base (the bundle is the $N$ th power of the Hopf map $\left.\mathcal{H}: \mathbf{S}^{3} \rightarrow \mathbf{S}^{2}\right)$. Hence $\delta C$ integrates to $\pi N$ over $L(1, N)$.

For simplicity, let us now specialise to the case $N=1$, where the geometry is smooth. If the unit three-sphere bundle of $N \widehat{W}$ is denoted $\Pi: \mathbf{S}^{3} \rightarrow \widehat{W}$, then we have

$$
\Pi_{*}(\delta C / \pi)=1
$$

That is, $\delta C / \pi$ integrates to 1 over a unit three-sphere fibre. Now, on any $n$-sphere bundle, there exists an $n$-form $\psi$, the global angular form [43], whose restriction to each fibre generates the cohomology of the fibre. This form also satisfies

$$
d \psi=-\Pi^{*} e
$$

That is, the exterior derivative is minus the pull-back of the Euler class of the spherebundle. Hence we may write

$$
\delta C / \pi=\psi+\ldots
$$

Since we know that $\delta C / \pi$ is a global form that integrates to 1 over each fibre, it follows that the terms "..." integrate to 0 over each fibre, and will not interest us. Thus, ignoring these for the moment, the shift in $C$ induces

$$
\delta G / 2 \pi=d \delta C / 2 \pi=\frac{1}{2} d \psi=-\Pi^{*} e(N \widehat{W}) / 2
$$

The pull-back of the Euler class to $N \widehat{W}$ will in general be non-trivial. We have thus shown that the half-integral contribution to $[G / 2 \pi]$ contributes a term given by the Euler class of the normal bundle, which ties in with the comments at the end of the previous subsection. In our case-studies, we have shown explicitly that this Euler class is equal to the restriction of $\lambda$ to $\widehat{W}$. Thus (5.19) reads

$$
\delta G / 2 \pi=-\lambda / 2
$$

Hence this K-theory shift is precisely the usual shift due to $\lambda$. We now go on to prove this in the general case, modulo two (which is all that matters). The reader may wish to skip the remainer of this subsection, which is rather technical, as the argument merely confirms previous results, rather than adding anything new.

Thus, reducing modulo two, and restricting to $\widehat{W}$, we obtain 


$$
[\delta G / \pi]=w_{4}(N \widehat{W}) \quad(\bmod 2)
$$

since for a rank four oriented vector bundle the Euler class reduces mod two to the fourth Stiefel-Whitney class. The Whitney product formula gives us

$w_{4}\left(\left.T M\right|_{\widehat{W}}\right)=w_{4}(T \widehat{W})+w_{1}(T \widehat{W}) w_{3}(N \widehat{W})+w_{2}(T \widehat{W}) w_{2}(N \widehat{W})+w_{3}(T \widehat{W}) w_{1}(N \widehat{W})+w_{4}(N \widehat{W})$

Now, $M^{11}$ is assumed spin, and so $w_{2}(T \widehat{W})=w_{2}(N \widehat{W}) . \widehat{W} \cong W$ is assumed orientable (in order to couple the D-brane to RR-forms). Hence $w_{1}(T \widehat{W})=0$. Moreover, $\widehat{W}$ must also be $\operatorname{spin}^{c}$, and so $w_{3}(T \widehat{W})=0$. We are left with

$$
\lambda\left(\left.T M\right|_{\widehat{W}}\right) \stackrel{\bmod 2}{=} w_{4}\left(\left.T M\right|_{\widehat{W}}\right)=w_{4}(T \widehat{W})+w_{2}(T \widehat{W})^{2}+w_{4}(N \widehat{W})
$$

Now, $\widehat{W}$ is an oriented $\operatorname{spin}^{c}$ six-manifold. Hence, arguing as we did in the last section, by Wu's Theorem

$$
w_{4}(\widehat{W})=\operatorname{Sq}^{1}\left(V_{3}\right)+\operatorname{Sq}^{2}\left(V_{2}\right)
$$

$V_{3}=0$ as $\widehat{W}$ is oriented and $\operatorname{spin}^{c}$, and $V_{2}=w_{2}(\widehat{W})$ as $w_{1}(\widehat{W})=0$. Since $\operatorname{Sq}^{2}\left(w_{2}\right)=$ $w_{2}^{2}$, we conclude that

$$
w_{4}(\widehat{W})+w_{2}^{2}(\widehat{W})=0
$$

Applying this to our formula above, we see that

$$
\lambda\left(\left.T M\right|_{\widehat{W}}\right)=w_{4}(N \widehat{W}) \quad(\bmod 2)
$$

We conclude that, in the general case, the K-theory shift we have found in this section is equivalent to the half-integral shift in $G$ found from M2-brane worldvolume anomalies.

\section{Effective $\mathcal{N}=1$ Three-Dimensional Field Theory}

In this section we discuss the effective three-dimensional field theory obtained from M-theory compactification on a (singular) manifold $X$ of $\operatorname{Spin}(7)$ holonomy. After discussing general aspects of such compactifications, we then go on to study in detail two examples obtained from compactification of M-theory on $\mathbb{M} \mathbb{B}_{8}$ and $\mathcal{Q}$. We describe

\footnotetext{
${ }^{19}$ In this section we use the notation $\mathbb{B}_{8} \equiv \Sigma_{-} \mathbf{S}^{4}$ to denote the total space of the bundle of negative chirality spinors over $\mathbf{S}^{4}$, as in 15 .
} 
some dynamical aspects of both these models and also explain the relation between the anomalous shift of the background $G$-flux and the Chern-Simons coefficient in the $\mathcal{N}=1$ three-dimensional effective field theory.

\subsection{Compactification on a General Spin(7) Manifold}

In general, the effective field theory is expected to be $\mathcal{N}=1$ supersymmetric gauge theory in three dimensions. If for a moment we assume that $X$ is non-singular (although it may still be non-compact) and gently curved, then one can deduce the spectrum of the massless modes from the Kaluza-Klein reduction of eleven-dimensional supergravity. As a result, in the compact case one finds a total number of $\left(b_{2}(X)+b_{3}(X)+b_{4}^{-}(X)+1\right)$ bosonic modes and the same number of fermionic modes, which complete the $\mathcal{N}=1$ supersymmetry multiplets [51]. In a theory without a superpotential or Chern-Simons couplings, all of these bosonic modes may be thought of as scalar fields (due to vectorscalar duality in three dimensions).

However, as will be explained below, many models typically have both Chern-Simons and superpotential terms, which prevents us from dualizing scalar and vector fields into each other. Therefore, we have to distinguish between vector modes and scalar field modes. Bearing this in mind, from the Kaluza-Klein reduction we find $b_{2}$ abelian vector fields, $\mathcal{A}^{i}$, which come from the modes of the three-form field $C$, and $\left(b_{3}+b_{4}^{-}+1\right)$ scalars, $\phi^{a}$. Some of these scalar fields, namely $b_{3}$ of them, come from the $C$-field, whereas the others correspond to deformations of the $\operatorname{Spin}(7)$ structure. If $X$ is noncompact, instead of the Betti numbers $b_{k}$ one should use the dimension of the space of $L^{2}$-normalisable $k$-forms on $X$.

Taking into account Chern-Simons and superpotential terms, we may write the complete supersymmetric action at a generic point in the moduli space of $X$ :

$$
\begin{aligned}
S_{3 d}=\int & d^{3} x\left[\sum_{k} \frac{1}{4 g_{k}^{2}}\left(\mathcal{F}_{\mu \nu}^{k} \mathcal{F}^{k \mu \nu}+\bar{\psi}^{k} i \Gamma \cdot D \psi^{k}\right)+\frac{1}{2} \sum_{a, b} g_{a b}\left(\partial_{\mu} \phi^{a} \partial^{\mu} \phi^{b}+\bar{\chi}^{a} i \Gamma \cdot D \chi^{b}\right)-\right. \\
& \left.-\frac{1}{2} \sum_{a, b} g^{a b}\left(\frac{\partial \mathcal{W}(\phi)}{\partial \phi^{a}} \frac{\partial \mathcal{W}(\phi)}{\partial \phi^{b}}-\frac{1}{2} \frac{\partial^{2} \mathcal{W}(\phi)}{\partial \phi^{a} \partial \phi^{b}} \bar{\chi}^{a} \chi^{b}\right)\right]-\sum_{i, j} \frac{i k_{i j}}{4 \pi} \int\left(\mathcal{A}^{i} \wedge d \mathcal{A}^{j}+\bar{\psi}^{i} \psi^{j}\right)
\end{aligned}
$$

Here, $\psi^{i}$ are the gaugino fields, $\chi^{a}$ represent the fermionic superpartners of the scalar fields $\phi^{a}, g^{i}$ are the gauge couplings, and $g^{a b}$ denotes the scalar field metric. Since we are mainly interested in non-compact $\operatorname{Spin}(7)$ manifolds, in this Lagrangian we omit the terms corresponding to interactions with supergravity.

If the space $X$ develops a singularity, one should also expect some non-abelian gauge fields coming from the singularity. In the models that admit a description in terms of $D 6$-branes - such as $X=\mathbb{B}_{8}$ and $X=\mathcal{Q}$ discussed in this paper - one can derive non-abelian degrees of freedom from the corresponding D-brane models. The effective 
action for the non-abelian fields can be written as $\operatorname{Tr}(\ldots)$. In particular, the ChernSimons terms take the form

$$
I_{\mathrm{CS}}=\frac{k}{4 \pi} \int \operatorname{Tr}\left(\mathcal{A} \wedge d \mathcal{A}+\frac{2}{3} \mathcal{A} \wedge \mathcal{A} \wedge \mathcal{A}+\bar{\psi} \psi\right)
$$

where $\mathcal{A}$ is a gauge connection in the adjoint representation of the gauge group.

Now, following [37, let us discuss vacua in the resulting theory, and domain walls which connect them. As we explained in section 3, microscopically a domain wall in the three-dimensional $\mathcal{N}=1$ field theory can be described as an M5-brane wrapped over a topologically non-trivial 4-cycle:

$$
\Sigma^{(4)} \in H_{4}(X ; \mathbb{Z})
$$

These domain walls, classified by elements of the homology group $H_{4}(X ; \mathbb{Z})$, interpolate between vacua corresponding to different values of the $G$-flux. The latter, in turn, are classified ${ }^{\mathbb{O}}$ by $H^{4}(X ; \mathbb{Z})$. On a compact manifold these two groups are isomorphic, by Poincaré duality, so that in such a model all vacua can be connected by domain walls.

In the present paper we are interested in the case of a non-compact space $X$, where Poincaré duality asserts that $H_{4}(X ; \mathbb{Z})$ is isomorphic to cohomology with compact support:

$$
H_{4}(X ; \mathbb{Z}) \cong H_{\mathrm{cpct}}^{4}(X ; \mathbb{Z})
$$

Then, from the long exact sequence for the pair $(X, \partial X)$ it follows that different vacua, modulo those which can be connected by domain walls, are classified by the cohomology of the boundary 7-manifold $Y=\partial X$ 37]:

$$
H^{4}(Y ; \mathbb{Z})=H^{4}(X ; \mathbb{Z}) / f\left(H_{\mathrm{cpct}}^{4}(X ; \mathbb{Z})\right)
$$

where $f\left(H_{c p c t}^{4}(X ; \mathbb{Z})\right)$ is the image of the cohomology with compact support under the natural map:

$$
f: H_{\mathrm{cpct}}^{4}(X ; \mathbb{Z}) \rightarrow H^{4}(X ; \mathbb{Z})
$$

Therefore, we conclude that different models are classified in part by $H^{4}(Y ; \mathbb{Z})$.

The other data needed to completely specify the compactification is the value of the flux at infinity [37:

$$
\Phi_{\infty}=N_{M 2}-\frac{\chi(X)}{24}+\frac{1}{2} \int \frac{G}{2 \pi} \wedge \frac{G}{2 \pi}
$$

Here $N_{M 2}$ is the number of membranes filling three-dimensional space-time, and $\chi(X)$ is the integral of the Euler density over $X$. Note also that the anomaly cancellation condition requires $\Phi_{\infty}=0$ for a compact space $X$ [52, 18].

\footnotetext{
${ }^{20}$ Again, we stress that it is the shifted $G$-flux (including the quantum correction due to $\lambda$ ) that is integral.
} 
If $X$ is non-compact, the $\chi(X)$ that enters the global anomaly condition (6.7) is defined as an integral of the Euler density over $X$. This may not agree with the topological Euler number of $X$. There is an effective way to compute $\chi(X)$, provided that an equivalent D6-brane model is available. Indeed, let us assume that M-theory on the $\operatorname{Spin}(7)$ manifold $X$ can be viewed as the lift of some D6-brane configuration on a $G 2$ space $M^{7}$ to eleven dimensions. Both of our models may be realised in this way with $M^{7}$ being either $M^{7}=\Lambda^{-} B$ or (topologically) $\mathbb{R}^{7}$. In the first case, a D6-brane is wrapped on the non-trivial coassociative 4-cycle ${ }^{21} B$ in the $G 2$ space $M^{7}=\Lambda^{-} B$, as in section 5 . In the second case, discussed in section 3, the D6-brane has world-volume $\mathbb{R}^{3} \times L \subset \mathbb{R}^{10}$. For simplicity, let us assume ${ }^{22}$ that $G=0$, so the anomaly condition (6.7) reads:

$$
\frac{\chi(X)}{24}=N_{M 2}-\Phi_{\infty}
$$

The reason we decided to write the anomaly condition in this form is that the righthand side of (6.8) represents the effective M2-brane charge, whereas the left-hand side is the anomaly term (obtained by integrating $R^{4}$ terms in the eleven-dimensional action). After reduction to Type IIA theory the effective membrane charges become the effective charge of space-filling D2-branes. What is the Type IIA interpretation of the left-hand side of the anomaly formula (6.8)?

Since from the Type IIA perspective the three-dimensional effective theory is obtained by compactification on a seven-dimensional $G 2$ manifold $M^{7}$, there is no contribution to the D2-brane charge from the bulk. However, in Type IIA theory we also have a space-filling D6-brane wrapped on the coassociative 4-cycle $B$ inside $M^{7}$. Due to the non-trivial embedding of the D6-brane world-volume in space-time, the Ramond-Ramond fields couple to the gauge field strength $\mathcal{F}$ as [36, 53, 54]:

$$
I_{\mathrm{WZ}}=\int_{\mathbb{R}^{3} \times B} C_{*} \wedge \operatorname{Ch}(\mathcal{F}) \wedge \sqrt{\frac{\hat{A}\left(T_{B}\right)}{\hat{A}\left(N_{B}\right)}}
$$

Here $T_{B}$ (respectively $N_{B}$ ) denotes the tangent (respectively normal) bundle of $B$ inside $M^{7}$ (not inside $X$ !), and the Dirac genus $\hat{A}$ can be expressed in terms of the Pontryagin classes as follows [55]:

$$
\hat{A}=1-\frac{p_{1}}{24}+\frac{7 p_{1}^{2}-4 p_{2}}{5760}+\ldots
$$

If we now compare the $C_{3}$ coupling on the right-hand side of (6.9) with the left-hand

\footnotetext{
${ }^{21}$ We remind the reader that the main two examples discussed in this paper correspond to $B=\mathbf{S}^{4}$ and $B=\mathbb{C P}^{2}$.

${ }^{22}$ Of course, this cannot occur in our models, but the conclusions are in any case independent of this assumption.
} 
side of the formula (6.8), we obtain a relation:

$$
\frac{\chi(X)}{24}=\int_{B} \operatorname{Ch}(\mathcal{F}) \wedge \sqrt{\frac{\hat{A}\left(T_{B}\right)}{\hat{A}\left(N_{B}\right)}}
$$

In particular, if the gauge bundle on the D6-brane is trivial, from the total D2-brane charge induced by the anomaly we find:

$$
\chi(X)=\frac{N}{2} \int_{B}\left(p_{1}\left(N_{B}\right)-p_{1}\left(T_{B}\right)\right)
$$

We should stress here that the right-hand side of this formula is computed on a $G 2$ manifold $M^{7}$, whereas the left-hand side is computed on the corresponding 8-manifold $X$ of Spin(7) holonomy. Thus, we are able to compute $\chi(X)$ (which in the compact case is the Euler number of $X$ ) by computing locally the two-brane charge which is induced on the D6-branes. In fact, a similar picture arises in F-theory 52. In the latter reference it was argued that the Euler number of a (non-singular) elliptically fibred Calabi-Yau four-fold could be computed by calculating the local three-brane charge which is induced on the seven-branes, the latter having a worldvolume given by the discriminant of the fibration (times flat Minkowski four-space). This three-brane charge is again induced by WZ terms involving Pontryagin classes, and therefore the situation described in the present paper may be regarded as an M-theory analogue of the F-theory picture outlined in [52].

Now, let us briefly mention the rôle of supersymmetry. In M-theory on a manifold $X$ of $\operatorname{Spin}(7)$ holonomy, vanishing of the supersymmetry variations of the gravitino fields implies that the covariantly constant spinor $\eta$ obeys [56, 15]:

$$
G_{m p q r} \gamma^{p q r} \eta=0
$$

This can be expressed in terms of the Cayley 4-form $\Phi$ :

$$
G_{m p q r} \Phi_{n}^{p q r}=0
$$

This condition implies that in a supersymmetric vacuum the $G$-flux must be self-dual:

$$
G=* G
$$

This explains, for example, why no solutions with anti-self-dual four-form flux have been found in [15]. Moreover, from the self-duality of $G$ and the anomaly condition (6.7) it follows that on a compact $\operatorname{Spin}(7)$ manifold there can be only finitely many supersymmetric vacua. In fact, we can rewrite (6.7) as:

$$
\Phi_{\infty}+\frac{\chi(X)}{24}=N_{M 2}+\frac{1}{2} \int \frac{G}{2 \pi} \wedge * \frac{G}{2 \pi}
$$


Since a given choice of the covariantly constant spinor $\eta$ is compatible with membranes of only one orientation 23 , supersymmetry also requires:

$$
N_{M 2} \geq 0
$$

It follows that in a supersymmetric configuration the right-hand side of (6.16) is always non-negative, and because the $G$-flux is quantised it can take only finitely many values, for $X$ compact. In particular, if $\chi(X)<0$ there are no supersymmetric vacua at all.

Note also that the right-hand side of $(6.16)$ can be interpreted as the energy density in a given vacuum. Since for a finite tension domain wall the energy densities in the two vacua connected by the wall should be the same, it follows that the sum of the two terms on the right-hand side of (6.16) is invariant in a given model (although individual terms are not), $c f$. [37. This is another way to argue that $\Phi_{\infty}$ is an invariant of the dynamics.

Finally, let us point out that, at least in the supergravity approximation, the position of the membranes is arbitrary in $X$, so that the three-dimensional effective theory has $8 \cdot N_{M 2}$ moduli (super)fields, which parametrise $N_{M 2}$ copies of $X$. After reduction to Type IIA theory these membranes turn into D2-branes, localised at arbitrary points of the internal space. However, if we have a configuration of multiple D6-branes, $N>1$, it is natural to consider a 'Higgs branch', where the D2-branes dissolve in the D6-branes. In fact, every such D2-brane looks like an instanton on $B$ in the world-volume theory of the D6-branes. Hence, $N_{M 2}$ dissolved D2-branes correspond to a configuration of $N_{M 2}$ instantons on $B$. Classically, scalar fields in the effective $\mathcal{N}=1$ three-dimensional theory parametrise the moduli space $\mathcal{M}_{N_{M 2}}(B)$, of $N_{M 2}$ instantons on $B$. In particular, the dimension of this branch is given by:

$$
\operatorname{dim}(\mathcal{M})=4 N_{M 2} N-\left(N^{2}-1\right)\left(1+b_{2}^{+}(B)\right)
$$

In our models we have $b_{2}^{+}\left(\mathbf{S}^{4}\right)=0$ and $b_{2}^{+}\left(\mathbb{C} \mathbf{P}^{2}\right)=1$.

This picture agrees nicely with the fact ${ }^{2}$ that the internal part of the D6-brane worldvolume theory is $\mathcal{N}=4$ topologically twisted Yang-Mills theory on the four-manifold $B$ [58. Specifically, it is the $\mathcal{N}=4$ topological theory such that the fundamental representation of the $S U(4)$ R-symmetry group decomposes into $(\mathbf{1}, \mathbf{2}) \oplus(\mathbf{1}, \mathbf{2})$ of the twisted $S U(2) \times S U(2)$ Lorentz group. This theory enjoys $S L(2, \mathbb{Z})$ duality symmetry [59]. In particular, one generator of this group corresponds to the shift of the background $C$-field:

$$
T: \int_{M^{3}} C \rightarrow \int_{M^{3}} C+2 \pi
$$

\footnotetext{
${ }^{23}$ Membranes with the opposite orientation can also be interpreted as anti-membranes.

${ }^{24}$ We thank N. Nekrasov for discussions on this point.
} 
This is in fact a symmetry of M-theory, since $G=d C$ is invariant under both local gauge transformations, $C \rightarrow C+d \nu$, with $\nu$ a two-form, and also "large" gauge transformations, $C \rightarrow C+\mu$, where $\mu$ is a closed three-form with $2 \pi$ periods. This latter transformation acts as follows on the coupling constant in the $\mathcal{N}=4$ topological theory:

$$
T: \tau \rightarrow \tau+1
$$

The partition function of this theory can be written as a series in $q=\exp (2 \pi i \tau)$ :

$$
Z=\mathrm{const} \sum_{n} \chi\left(\mathcal{M}_{n}(B)\right) q^{n}
$$

It would be interesting to study further the relation between this topological theory and the $\mathcal{N}=1$ effective theory in three dimensions.

In the rest of this section we will mainly be interested in three-dimensional vacua which have a mass gap. So, we assume that $N_{M 2}=0$, unless otherwise stated.

To summarise, the models with mass gap may be microscopically classified by the value of $\Phi_{\infty}$, and by $[G / 2 \pi]$ in a given coset (6.5), so that (6.7) is obeyed.

\subsection{Chern-Simons Terms Induced by G-Flux}

As we have mentioned earlier, M-theory on a Spin(7) manifold $X$ can often be understood as the lift of a certain D6-brane configuration, and under such a duality some non-abelian degrees of freedom are mapped to gauge fields on multiple D-branes. Thus, in both of our examples we can start with $N$ D6-branes wrapped on the coassociative 4-cycle $B$ in the manifold $M^{7}=\Lambda^{-} B$ of $G 2$ holonomy. Clearly, in both cases, corresponding to $B=\mathbf{S}^{4}$ and $B=\mathbb{C P}^{2}$, we obtain a $U(N)$ factor in the three-dimensional gauge group 20 .

In three dimensions, in addition to the usual Maxwell-Yang-Mills term, one may also include in the Lagrangian a level $k$ supersymmetric Chern-Simons term:

$$
I_{\mathrm{CS}}=\frac{k}{4 \pi} \int \operatorname{Tr}\left(\mathcal{A} \wedge d \mathcal{A}+\frac{2}{3} \mathcal{A} \wedge \mathcal{A} \wedge \mathcal{A}+\bar{\psi} \psi\right)
$$

Our goal in this subsection will be to understand how such terms can be generated by classical and quantum effects in M-theory.

The Chern-Simons coupling $k$ is quantised topologically 60. However, integrating out the gluino fields $\psi$ generates a shift in the effective value of $k$. This shift is exact

\footnotetext{
${ }^{25}$ In the case of $B=\mathbb{C} \mathbf{P}^{2}$ one finds an extra $U(1)$ factor from the bulk fields. It will be discussed in section 6.4 .
} 
at one loop. Specifically, the level is shifted to [62, 61]:

$$
k_{\text {eff }}=k-\frac{N}{2} \operatorname{sign}(k)
$$

for gauge group $S U(N)$. The level $k_{U(1)}$ of the $U(1)$ gauge factor is not renormalised, i.e. $k_{\mathrm{eff}}=k$.

It is $k_{\text {eff }}$ that is now quantised in a purely bosonic effective field theory. We may as well assume that $k>0$, and so the quantisation condition becomes:

$$
k-\frac{N}{2} \in \mathbb{Z}
$$

Hence, for $N$ odd, the shift in $k$ is half-integral, and, in particular, $k$ cannot be zero. Since the theory conserves parity only for $k=0$, this is often called the parity anomaly [63, 64, 18].

In order to see how such Chern-Simons terms can be generated by the background $G$-flux in our models, notice that on the worldvolume of the D6-branes, one has the following Wess-Zumino term [36]:

$$
I_{\mathrm{WZ}}=\int_{M^{3} \times B} \operatorname{Ch}(\mathcal{F}) \wedge C_{*}=\frac{1}{2} \int_{M^{3} \times B} \operatorname{Tr}\left(\frac{\mathcal{F}}{2 \pi} \wedge \frac{\mathcal{F}}{2 \pi}\right) \wedge C_{3}
$$

where $C_{3}$ is the pull-back to $M^{3} \times B$ of the RR three-form potential, and $\mathcal{F}$ is the curvature of the gauge field on the branes. Here $B$ is either the $\mathbf{S}^{4}$ or $\mathbb{C} \mathbf{P}^{2}$ bolt of $\mathbb{B}_{8}$ or $\mathcal{Q}$, respectively. Integrating by parts, we have:

$$
I_{\mathrm{WZ}}=\frac{1}{4 \pi} \int_{M^{3} \times B} \operatorname{Tr}\left(\mathcal{A} \wedge d \mathcal{A}+\frac{2}{3} \mathcal{A} \wedge \mathcal{A} \wedge \mathcal{A}\right) \wedge \frac{G_{4}}{2 \pi}
$$

Integrating over $B$ and taking into account the fermionic superpartners of $\mathcal{A}$, we obtain the following Chern-Simons term in the effective supersymmetric gauge theory:

$$
I_{\mathrm{CS}}=\frac{k}{4 \pi} \int_{M^{3}} \operatorname{Tr}\left(\mathcal{A} \wedge d \mathcal{A}+\frac{2}{3} \mathcal{A} \wedge \mathcal{A} \wedge \mathcal{A}+\bar{\psi} \psi\right)
$$

where:

$$
k=\int_{B} \frac{G}{2 \pi}
$$

Note that $k$ does not have to take integer values. It is $k_{\text {eff }}$ which should always be integer, and the corresponding value of $k$ takes either integer or half-integer values in order to produce a consistent model.

The world-volume theory on a D6-brane contains fermions, which might also cause a shift in the Chern-Simons level. In fact, integrating out a massive fermion of mass $M$ 
in a representation $R$ of the gauge group, one finds a finite one-loop renormalisation 62:

$$
k \rightarrow k+\frac{d_{2}(R)}{2} \operatorname{sign}(M)
$$

where $d_{2}(R)$ is the quadratic Casimir of $R$. This is precisely what happens in the Type IIB supergravity dual of $\mathcal{N}=1$ three-dimensional gauge theory, proposed recently by Maldacena and Nastase [10]. Specifically, the model of [10] consists of $N$ five-branes in Type IIB theory wrapped on $\mathbf{S}^{3}$. As in the present setup, there is a certain amount of $H$ flux through the three-sphere, which via the Wess-Zumino coupling (6.25) determines the Chern-Simons level in the effective theory on the D5-branes. The value of the $H$-flux is not shifted, but there is still a finite renormalisation of $k$ that comes from summing over all massive chiral multiplets. In fact, as shown in [10], all the massive fermionic modes on $\mathbf{S}^{3}$ have partners with opposite mass, except for one mode, which leads to the finite shift in $k$. In our case, there is no contribution like (6.29) from massive fermions on the four-dimensional manifold $B$ since all the modes with positive and negative mass are paired up 20 .

Now, let us focus on the case $N=1$, where the geometry of $X$ is smooth, and the effective theory in three dimensions is abelian. From the last section, we know that the flux of the $G$-field in M-theory is shifted from standard Dirac quantisation 27 :

$$
k_{0}=\int_{B}\left(\frac{G}{2 \pi}-\frac{\lambda}{2}\right) \in \mathbb{Z}
$$

This shift results in a shift of the Chern-Simons coefficient in the three dimensional gauge theory:

$$
k=k_{0}+\delta k
$$

where

$$
\delta k=\int_{B} \frac{\lambda}{2}
$$

In both of the cases $X=\mathbb{B}_{8}$ and $X=\mathcal{Q}, k_{0}$ is therefore shifted to $k=k_{0}+1 / 2$ by quantum corrections in M-theory. However, since the three-dimensional gauge theory is abelian, we have:

$$
k_{\text {eff }}=k=k_{0}+\frac{1}{2}
$$

\footnotetext{
${ }^{26}$ We thank J. Maldacena for explanations and very helpful discussions on these points.

${ }^{27}$ Note that we have three different $k \mathrm{~s}$ here. The value of $k_{0}$ stands for the starting value of the $G$-flux in the eleven-dimensional supergravity theory (without quantum correction $\lambda$ ), whereas $k$ and $k_{\text {eff }}$ denote the Chern-Simons coefficients in supersymmetric three-dimensional theory and its bosonic low-energy description, respectively.
} 
At first sight this might seem to be a contradiction because $k_{\text {eff }}$ is half-integer for integer $k_{0}$. However, a special property of abelian Chern-Simons theory - that string theory seems to know about - is that it can be consistently defined for both integer and half-integer values of $k$. Moreover, it can be consistently defined on any closed oriented three-dimensional manifold $M^{3}$ [65].

The construction may be described as follows [65]. Let us start with an arbitrary closed oriented three-dimensional manifold $M^{3}$ (so far, we mainly focused on the cases where $M^{3}=\mathbb{R}^{3}$ or $M^{3}=T^{3}$ ). Since any oriented three-dimensional manifold is spin, we can consider $\mathcal{N}=1$ supersymmetric gauge theory on $M^{3}$. As before, let $\mathcal{F}$ denote the curvature on the $U(1)$ gauge bundle $\mathcal{L}$ over $M^{3}$. Such bundles are classified by maps to $\mathbb{C P}^{\infty}$. As pointed out in [65], the spin bordism group is

$$
\Omega_{3}^{\mathrm{Spin}}\left(\mathbb{C} \mathbf{P}^{\infty}\right)=0
$$

This implies that both the spin structure and the gauge bundle $\mathcal{L}$ may be extended over some oriented four-manifold $M^{4}$ with boundary $M^{3}$. One can then define the level $k$ Chern-Simons term as [65]:

$$
I_{\mathrm{CS}}=\frac{k}{4 \pi} \int_{M^{4}} \mathcal{F} \wedge \mathcal{F}
$$

A priori, this definition depends on the choice of four-manifold, $M_{4}$. However, suppose that one may find another such four-manifold $M_{4}^{\prime}$. One may glue $M_{4}$ and $M_{4}^{\prime}$ along their common boundary $M_{3}$

$$
Y_{4} \equiv M_{4} \bigcup_{M_{3}}-M_{4}^{\prime}
$$

to form the closed four-manifold $Y_{4}$. It follows that the difference between the ChernSimons terms defined by $M_{4}$ and $M_{4}^{\prime}$ is given by

$$
\frac{k}{4 \pi} \int_{Y_{4}} \mathcal{F} \wedge \mathcal{F}
$$

Since $Y_{4}$ is spin, the intersection form on $H^{2}\left(Y_{4} ; \mathbb{Z}\right)$ is even, and hence this last formula is always an integral multiple of $4 \pi k$. Hence, $I_{\mathrm{CS}}$ defined by (6.35) is welldefined, up to the addition of some multiple of $2 \pi$, even for $k \in \mathbb{Z}+\frac{1}{2}$. It follows that the path-intergral factor $e^{i I_{\mathrm{CS}}}$ is well-defined. This ensures consistency of Type IIA Dbrane configurations dual to M-theory on the $\operatorname{Spin}(7)$ manifolds $\mathbb{B}_{8}$ and $\mathcal{Q}$ disscussed in the present paper. In both models one finds Chern-Simons terms at half-integer level. Notice that the definition of the Chern-Simions term, $I_{\mathrm{CS}}$ with $k \in \mathbb{Z}+1 / 2$, depends on the choice of a spin structure on $M^{3}$. 
Let us now look at the M-theory picture. The presence of fluxes in M-theory or string theory typically leads to superpotential and Chern-Simons terms in the lowerdimensional theory [37, 66]. The explicit expression for the Chern-Simons coupling can be derived from the analogous Chern-Simons term in the eleven-dimensional effective action

$$
\frac{I_{C S}^{(11)}}{2 \pi}=\frac{1}{6 \cdot(2 \pi)^{3}} \int C \wedge G \wedge G
$$

In flat space, the $U(1)$ gauge field on the D6-branes derives from a Kaluza-Klein reduction of the M-theory three form potential $C$ along the $L^{2}$-normalisable harmonic two-form of Taub-NUT space, $\omega^{(2)}$. Thus one makes an ansatz of the form $C=$ $\mathcal{A} \wedge \omega^{(2)}$, which gives rise to a dynamical gauge field $\mathcal{A}$ upon reduction. Since the eight-manifolds of $\operatorname{Spin}(7)$ holonomy discussed here also represent the M-theory lift of D6-branes in Type IIA theory, one would expect them to possess $L^{2}$-normalisable exact harmonic two-forms, in order to reproduce the correct spectrum of gauge fields in three dimensions. However, as in [45], we find that exact harmonic two-forms $\omega^{(2)}$ do indeed exist on these spaces, but they are not $L^{2}$-normalisable. This would imply that the corresponding gauge fields are non-dynamical.

Bearing this puzzle in mind, in Type IIA theory each D6-brane carries a $U(1)$ gauge field $\mathcal{A}$, which corresponds to a Kaluza-Klein mode of the $C$-field in M-theory, $C=$ $\mathcal{A} \wedge \omega^{(2)}$. In the case of several harmonic two-forms we obtain

$$
C=\sum_{i} \mathcal{A}_{i} \wedge \omega_{i}^{(2)}
$$

Substituting this into (6.38) and integrating over the eight-manifold $X$, we get

$$
I_{C S}=\frac{k_{i j}}{4 \pi} \int_{\mathbb{R}^{3}} \mathcal{A}_{i} \wedge d \mathcal{A}_{j}
$$

where the Chern-Simons coefficients, $k_{i j}$, are given by:

$$
k_{i j}=\int_{X} \frac{G}{2 \pi} \wedge \omega_{i}^{(2)} \wedge \omega_{j}^{(2)}
$$

For example, in the case $X=\mathbb{B}_{8}=\Sigma_{-} \mathbf{S}^{4}$ we expect a single harmonic two-form $\omega^{(2)}$, whose square determines the anomalous shift in $k$ :

$$
\delta k=\frac{1}{2} \int_{X} \lambda \wedge \omega^{(2)} \wedge \omega^{(2)}
$$

One would like to reconcile this formula with the Type IIA result (6.32). In order to see this relation, consider for example the ALC $\operatorname{Spin}(7)$ metric (2.5). This describes 
the uplift of a D6-brane wrapped on the $\mathbf{S}^{4}$ of $\Lambda^{-} \mathbf{S}^{4}$, with an asymptotically finite value of the string coupling constant. This solution has a $U(1)$ Killing vector field, $\partial / \partial \phi$, that generates rotations of the asymptotically finite sized $\mathbf{S}^{1}$. As is well-known, on a Ricci-flat manifold the one-form dual to such a $U(1)$ Killing vector is harmonic. Specifically, we may write

$$
\eta^{(1)}=f^{2}(r) \sigma
$$

and

$$
\omega^{(2)}=d \eta^{(1)}
$$

where the function

$$
f^{2}(r)=\frac{(r-3 a)(r+a)}{(r-a)^{2}}
$$

and $\sigma=d \phi+A$ where $A$ is a connection on the $U(1)$ bundle. It is easy to verify that $\eta^{(1)} / 2 \pi$ is harmonic, and integrates to one over the $\mathbf{S}^{1}$ at infinity. Indeed, $\sigma / 2 \pi$ may be regarded as a global angular form on the $U(1)$ bundle over the twistor space $\mathbb{C P}^{3}$ of $\mathbf{S}^{4}$. The total space of this bundle is of course diffeomorphic with the boundary of $\mathbb{B}_{8}$. The form $\sigma$ is only defined on the complement of the zero section of $\Sigma_{-} \mathbf{S}^{4}$, but the form $\eta^{(1)}$ is in fact a global form on $\mathbb{B}_{8}$ since $f^{2}(r)$ vanishes at the zero-section $r=3 a$. Moreover, the derivative of $\sigma$ is the curvature $F$ of the $U(1)$ bundle. When projected down to Type IIA, this by definition means that $d \sigma$ coincides with the RR two-form $G_{2}$. The formula $(6.42)$ therefore gives

$$
\begin{aligned}
\delta k & =\frac{1}{2} \int_{X} \lambda \wedge d \eta^{(1)} \wedge \omega^{(2)} \\
& =\frac{1}{2} \int_{\partial X} \lambda \wedge \eta^{(1)} \wedge \omega^{(2)} \\
& =\frac{1}{2} \int_{\partial X / U(1)} \lambda \wedge \frac{G_{2}}{2 \pi} \\
& =\frac{1}{2} \int_{\mathbf{S}^{4}} \lambda
\end{aligned}
$$

where in the last step we have used the fact that $G_{2} / 2 \pi$ integrates to one over the $\mathbf{S}^{2}$ fibre of the twistor space $\mathbf{S}^{2} \hookrightarrow \mathbb{C} \mathbf{P}^{3} \rightarrow \mathbf{S}^{4}$, since there is one D6-brane present. Hence we recover the Type IIA formula (6.32). Precisely the same reasoning goes through for the case $X=\mathcal{Q}$, with the new $\operatorname{Spin}(7)$ metric (4.54). 
In fact, with some care this argument may be used to determine the shift in $k$ for $N>1$. It is natural to expect that in the case of non-abelian gauge theory $\delta k$ is related to the one-loop shift of $k$ in the IR bosonic theory, (6.23). However, for $N>1$ the M-theory "manifold" is singular; specifically, it contains a $\mathbb{Z}_{N}$ orbifold singularity at the D6-brane locus. Thus one must be careful when using the results of [18] to determine the shift in the quantisation of the $G$-flux. However, in the smooth case, we know that the form $G / 2 \pi$ gets shifted by

$$
\frac{1}{16 \pi^{2}} \operatorname{Tr} R \wedge R
$$

where $R$ is the curvature of some smooth metric on $X$. The cohomology class of this differential form is associated with the integral characteristic class $\lambda(X)=p_{1}(X) / 2$ of the tangent bundle of $X$.

Now suppose that we take a $\mathbb{Z}_{N}$ quotient of $X$, such that the fixed point set is a codimension four submanifold of $X$. This is identified with the D6-brane locus in Type IIA. If we denote the complement of the fixed point set as $X_{0}$, then $X_{0} / \mathbb{Z}_{N}$ is a smooth manifold, and we are free to use the quantisation law for $G$ found in [18]. That is, $G / 2 \pi$ is shifted in the quantum theory by the gravitational correction term (6.47). Thus it would seem natural that the shift in $G / 2 \pi$ as a differential form on $X_{0} / \mathbb{Z}_{N}$ is the same as it is for $N=1$. We may therefore perform the integration just as for $N=1$ :

$$
\begin{aligned}
\delta k & =\frac{1}{2} \int_{\partial X / U(1)} \frac{1}{16 \pi^{2}} \operatorname{Tr}(R \wedge R) \wedge \frac{G_{2}}{2 \pi} \\
& =\frac{N}{2} \int_{\mathbf{S}^{4}} \frac{1}{16 \pi^{2}} \operatorname{Tr}(R \wedge R) \\
& =\frac{N}{2}
\end{aligned}
$$

Here we have used the fact that the RR two-form field strength $G_{2} / 2 \pi$ integrates to $N$ over the two-sphere fibre of the twistor space. This is equivalent to the assertion that there are $N$ D6-branes present. Thus this argument would seem to imply that the shift in quantisation of $G$ is indeed related to the one-loop shift in the value of the Chern-Simons coupling. It would be interesting to investigate this further [20].

Apart from the Chern-Simons term, the background $G$-flux also generates an effective superpotential, therefore giving a mass to the scalar fields. In M-theory compactification on a manifold $X$ of $\operatorname{Spin}(7)$ holonomy, one can construct a $\operatorname{Spin}(7)$ singlet by taking a wedge product of the $G$-flux with the Cayley calibration $\Phi$ :

$$
\mathcal{W}=\frac{1}{2 \pi} \int_{X} \Phi \wedge G
$$


It is natural to identify this function of the moduli fields with the effective superpotential induced by the $G$-flux 66]. Indeed, one can show that the supersymmetry constraints, which follow from the effective superpotential $\mathcal{W}$ are equivalent to the supersymmetry conditions (6.14) in the eleven-dimensional supergravity 67.

The combined effect of the Chern-Simons and superpotential terms is to produce a mass for the vector and scalar fields, and also to lift some vacua in the three-dimensional effective theory. Note that without the Chern-Simons and superpotential terms, i.e. in a theory where bosonic fields are massless, one can dualise vector fields (respectively multiplets) into scalar fields (respectively multiplets), and vice versa. Since we find that M-theory on either $\mathbb{B}_{8}$ or $\mathcal{Q}$ does not lead to propagating scalar fields in three dimensions, we will not discuss superpotential terms further in this paper.

\subsection{M-Theory on $\mathbb{B}_{8}$}

In this subsection we decribe some general features of the model based on $X=\mathbb{B}_{8}=$ $\Sigma_{-} \mathbf{S}^{4}$, and its $\mathbb{Z}_{N}$-quotient, corresponding to $N$ D6-branes in Type IIA string theory on $M^{7}=\Lambda^{-} \mathbf{S}^{4}$. A more complete treatment of the dynamics will be presented in [20]. The asymptotically locally conical $\operatorname{Spin}(7)$ metric (2.5) relevant to this model was found recently in [15], and reviewed in section 2 of the present paper.

We would like to study M-theory in the case when $X$ develops an isolated conical singularity. Since we are interested in the behaviour near the singularity, for the main part of this section we can think of $X$ as a smooth eight-manifold asymptotic to a cone over a seven-sphere:

$$
Y=S O(5) / S O(3) \cong \mathbf{S}^{7}
$$

The relevant $\mathrm{AC} \operatorname{Spin}(7)$ metric is given by 11 :

$$
d s^{2}=\left(1-\left(\frac{a}{r}\right)^{10 / 3}\right)^{-1} d r^{2}+\frac{9}{100} r^{2}\left(1-\left(\frac{a}{r}\right)^{10 / 3}\right)\left(\sigma_{i}-A^{i}\right)^{2}+\frac{9}{20} r^{2} d \Omega_{4}^{2}
$$

As the parameter $a \rightarrow 0, X$ develops an isolated conical singularity at $r=0$. A small deformation of the metric $\delta g / g \sim r^{-10 / 3}$ (corresponding to a change in the parameter a) is not $L^{2}$-normalizable since the following norm

$$
|\delta g|^{2}=\int d^{8} x \sqrt{g} g^{a c} g^{b d} \delta g_{a b} \delta g_{c d} \sim \int d^{8} x \sqrt{g}(\delta g / g)^{2} \sim \int d r r^{1 / 3}
$$

diverges. This means that the corresponding scalar field has zero kinetic energy and, therefore, its dynamics is frozen. In this sense, it is a true modulus (or, rather, a coupling constant). For each expectation value of this field, the models in question have finitely many vacua. 
Another way to see that the scalar mode corresponding to the change in the parameter $a$ is non-dynamical is to remember that compactification of M-theory on $X$ is equivalent to Type IIA string theory on a $G 2$ manifold $M^{7}=\Lambda^{-} \mathbf{S}^{4}$ with a D6-brane wrapped around the coassociative cycle $B \cong \mathbf{S}^{4}$. In this picture, the effective $\mathcal{N}=1$ three-dimensional theory is the theory on a space-filling D6-brane. In particular, light scalar fields come from normal deformations of the D6-brane world-volume inside the G2 manifold. According to McLean [22], such deformations of a coassociative submanifold $B$ are unobstructed and correspond to harmonic anti-self-dual two-forms on $B$. Moreover, he proves that the moduli space of coassociative manifolds is locally a smooth manifold of dimension:

$$
b_{2}^{-}(B)=\operatorname{dim} H_{-}^{2}(B ; \mathbb{R})
$$

Since $b_{2}^{-}\left(\mathbf{S}^{4}\right)=0$, we conclude that the coassociative 4 -sphere is rigid in this example and, therefore, that there are no light scalar fields on the D6-brane world-volume.

Let us now examine the global symmetries of the simplest $N=1$ model. As explained in [2], global symmetries that come from the $C$-field are gauge transformations $\delta C=d \Lambda$ that have $d \Lambda=0$ at infinity. Hence these transformations are classified by the group

$$
H^{2}(Y ; U(1))
$$

Moreover, unbroken symmetries are those which extend over $X$. In other words, unbroken symmetries are classified by

$$
H^{2}(X ; U(1))
$$

In the present example both (6.54) and (6.55) are trivial. So, we proceed to examine the geometrical symmetries.

Since $Y$ can be realised as a homogeneous space (6.50) of the form $G / H$, geometrical symmetries of $Y$ consist of left actions by the elements of $G$, and also from right actions by elements $w \in G$ that centralise $H$ :

$$
w^{-1} h w \in H, \quad \forall h \in H
$$

Since $S O(5)=S p(2) / \mathbb{Z}_{2}$ and $S O(3)=S p(1) / \mathbb{Z}_{2}$, the space $Y$ may also be viewed as the following quotient:

$$
Y=S p(2) / S p(1)
$$

For the natural action of $S p(1)$ on $S p(2)$ the induced Einstein metric is that of the "round" seven-sphere. However, this is not the metric we want. The metric on $Y$ relevant to our problem is a weak $G 2$ metric (since we require the cone on $Y$ to be 
Spin(7)), and is often described as a squashed seven-sphere. This may be constructed explicitly as follows. Consider $S p(1)_{A} \times S p(1)_{B} \subset S p(2)$, and define $G=S p(2) \times$ $S p(1)_{C}$, together with the subgroups $K=S p(1)_{A} \times S p(1)_{B} \times S p(1)_{C}$ and $H=S p(1)_{D} \times$ $S p(1)_{B}$, where $S p(1)_{D}=S p(1)_{A+C}$ is the diagonal subgroup of $S p(1)_{A} \times S p(1)_{C}$. Then the four-cycle $B$ is given by

$$
B=G / K
$$

and we can represent our seven-manifold $Y$ as:

$$
Y=G / H
$$

It follows that $Y$ fibres over $B$. The fibres themselves are copies of $K / H \cong S p(1) \cong$ $S U(2) \cong \mathbf{S}^{3}$. This is of course the quaternionic Hopf fibration. The induced metric on $Y$ is a weak $G 2$ metric and is in fact the squashed seven-sphere that we need. Up to homothety, the metric is given explicitly by

$$
d s_{7}^{2}=\mu^{2}\left(\sigma_{i}-A^{i}\right)^{2}+d \Omega_{4}^{2}
$$

where the "squashing parameter" $\mu^{2}=1 / 5$ for the weak G2 squashed seven-sphere (whereas the round sphere is given by $\mu^{2}=1$ ). This is to be compared with the $\operatorname{Spin}(7)$ metric (6.51), which may be regarded as a resolution of the cone over this weak G2 manifold. We briefly remind the reader that $d \Omega_{4}^{2}$ is the round metric on the unit $\mathbf{S}^{4}$, $\sigma_{i}$ are a set of left-invariant one-forms on $S U(2) \cong \mathbf{S}^{3}$, and $A_{i}$ is a connection for the $S U(2)$ Yang-Mills instanton on $\mathbf{S}^{4}$. Another way of realising the metric (6.60) is as the distance sphere in $\mathbb{H} \mathbf{P}^{2}$. That is, one takes a point in the quaternionic projective space $\mathbb{H} \mathbf{P}^{2}$ with its Fubini-Study metric, and considers the hypersurface consisting of all points a geodesic distance $\chi$ from that point, where $\mu=\cos \chi$. Clearly, for small $\chi$, the resulting hypersurface is topologically $\mathbf{S}^{7}$; the induced metric is given by (6.60), up to homothety.

Now we are ready to identify the symmetries of this metric. Since $Y$ may be viewed as the coset space (6.59), it is manifestly invariant under the left action of $G=S p(2) \times$ $S p(1)_{C}$. There are also symmetries of $Y$ that come from the right action by elements $w \in G$ that centralise $H$. In this case, there is only one such non-trivial element. It may be constructed as follows. Consider the following element of $S U(2) \cong S p(1)$

$$
\omega \equiv\left(\begin{array}{cc}
0 & 1 \\
-1 & 0
\end{array}\right)
$$

Then $w \equiv\left(\omega, 1, \omega^{-1}\right) \in S p(1)_{A} \times S p(1)_{B} \times S p(1)_{C}=K \subset G$. Notice that since $\omega^{-1}=-\omega, w$ is not an element of $H$. The action $w^{-1} H w$ simply complex conjugates 
the first copy of $S p(1)$ in $H=S p(1)_{D} \times S p(1)_{B}$. Notice that $w$ acts trivially on $B=\mathbf{S}^{4}$ since $w \in K$. We conclude that the symmetry group of $Y$ is

$$
\left[(S p(2) \times S U(2)) / \mathbb{Z}_{2}\right] \times \mathbb{Z}_{2}
$$

with the last factor generated by $w$. It is clear that this symmetry group extends to the resolution $X$ given by the $\operatorname{Spin}(7)$ metric (6.51), and hence there is no spontaneous symmetry breaking of this type.

Let us now examine the Type IIA dual of this solution, where a D6-brane is wrapped on the coassociative four-cycle $B=\mathbf{S}^{4}$ inside the $G 2$ holonomy manifold

$$
M^{7}=\Lambda^{-} \mathbf{S}^{4}
$$

From this perspective it is clear that the three-dimensional effective theory on the D6-brane is simply $\mathcal{N}=1$ supersymmetric $U(1)$ gauge theory with a Chern-Simons term:

$$
L_{3 d}=\frac{1}{4 g^{2}} \int d^{3} x\left(\mathcal{F}_{\mu \nu} \mathcal{F}^{\mu \nu}+\bar{\psi} i \Gamma \cdot D \psi\right)+\frac{i\left(k_{0}+\frac{1}{2}\right)}{4 \pi} \int(\mathcal{A} \wedge d \mathcal{A}+\bar{\psi} \psi)
$$

As we discussed in the previous subsection, the value of the Chern-Simons coefficient $k=k_{0}+1 / 2$ is half-integer and defined by the $G$-flux through the 4 -sphere, $c f .(6.30)$ :

$$
\int_{\mathbf{S}^{4}}\left[\frac{G}{2 \pi}\right]=k_{0}+\frac{1}{2}
$$

On the other hand, the gauge coupling constant $g$ is related to the volume of $\mathbf{S}^{4}$ :

$$
\frac{1}{g^{2}}=\operatorname{Vol}\left(\mathbf{S}^{4}\right) \sim a^{4}
$$

Of course, since the Chern-Simons coefficient $k$ is half-integer, in particular it cannot be zero. This implies that parity symmetry is broken in this theory. In a sense, this breaking of parity symmetry is spontaneous. Classically, one may turn off the ChernSimons term, so that the theory is parity-invariant. However, in M-theory one is forced to choose a physical state in which $G$ is non-zero, and therefore parity is violated in the effective three-dimensional theory due to this choice of state, rather than explicitly. Of course, even classically one may choose $G$ non-zero, and therefore violate parity, but the point is that in the quantum theory this is unavoidable.

There is another useful D6-brane configuration dual to this model, which was discussed in section 3. It is obtained from M-theory on $X$ via reduction on a circle $\mathbf{S}^{1} \cong U(1)$, such that $X / U(1) \cong \mathbb{R}^{7}, Y / U(1) \cong \mathbf{S}^{6}$, and the $U(1)$ action has a fixed 
point set in codimension four. Following [2], we denote $F \subset Y$ and $L \subset X$ to be the set of fixed points on $Y$ and $X$, respectively. Then, according to our analysis in section 3, $F$ must be a homology 3 -sphere, knotted inside $Y \cong \mathbf{S}^{\mathbf{7}}$. The fixed point set $F$ is isomorphic to an $\mathbf{S}^{1}$ bundle over $\mathbf{S}^{2}$ (with Euler number 1), so that $L$, which is a cone on $F$, is an $\mathbb{R}^{2}$ bundle over $\mathbf{S}^{2}$ :

$$
L \cong \mathbf{S}^{2} \times \mathbb{R}^{2}
$$

The singular configuration, where the $\mathbf{S}^{2}$ zero-section shrinks to zero size, corresponds to a point in the moduli space where $X$ develops a conical singularity. At this point the four-manifold $L$ degenerates to $\mathbb{R}^{4}$.

For each value of $k_{0}$ the theory is expected to be infrared-free and have only one massive vacuum. To see how this follows from M-theory, note that $H^{4}(Y ; \mathbb{Z})$ is trivial. Therefore, one would expect the model to be completely specified by the value of the flux at infinity (6.7):

$$
\Phi_{\infty}=\frac{1}{12}+\frac{1}{2}\left(k_{0}+\frac{1}{2}\right)^{2}
$$

Here we used the relation (6.12) to compute $\chi(X)=(-4-0) / 2=-2$, and also the fact that the self-intersection number of the $\mathbf{S}^{4}$ is equal to 1 . Note that the value of $k_{0}$ in the formula for $\Phi_{\infty}$ is related to the value of $k_{0}$ in the Chern-Simons term because the Chern-Simons coupling is induced by the $G$-flux, according to our discussion in the previous subsection. One can check that when $k_{0}$ is shifted by an integer, $\Phi_{\infty}$ also changes by an integer.

We have just explained that M-theory predicts only one supersymmetric vacuum in the $U(1)$ Chern-Simons theory on $\mathbb{R}^{3}$ at half-integer level. In particular, it does not depend on the value of the Chern-Simons coefficient. This is to be compared with the number of ground states in the $U(1)$ Chern-Simons theory, say, on $\mathbb{R} \times T^{2}$ at integer level $k$. Let us call this number $I^{\prime}(k)$. Then, $I^{\prime}(k)$ can be computed, for example, via quantisation of ground states 28 as in [9]:

$$
I^{\prime}(k)=|k|
$$

Note that the supersymmetric index in the $U(1)$ theory is equal to zero due to the presence of the fermionic zero mode. Excluding the fermionic zero mode one can

\footnotetext{
28 After we reduce the $2+1$-dimensional theory on $T^{2}$, we obtain supersymmetric quantum mechanics on $E$, where $E=\left(T^{2}\right)^{\vee}$ is the moduli space of flat $U(1)$ connections on $T^{2}$. The quantum Hilbert space of this model is the space of spinors with values in $\mathcal{L}^{k}$, or equivalently the space of $(0, q)$-forms valued in $\mathcal{L}^{k}$. Moreover, the supersymmetry generators can be identified with the operators $\bar{\partial}$ and $\bar{\partial}^{\dagger}$, whereas the Hamiltonian can be identified with $H=\left\{\bar{\partial}, \bar{\partial}^{\dagger}\right\}[9]$. It follows that supersymmetric ground states correspond to the elements of $H^{0}\left(E, \mathcal{L}^{k}\right) \oplus H^{1}\left(E, \mathcal{L}^{k}\right)$. Without loss of generality we may assume $k>0$, and, using the Riemann-Roch formula and Serre duality, compute: $I_{U(1)}^{\prime}(k)=h^{0}\left(\mathcal{L}^{k}\right)+h^{1}\left(\mathcal{L}^{k}\right)=\operatorname{deg}\left(\mathcal{L}^{k}\right)+2 h^{1}\left(\mathcal{L}^{k}\right)=k+2 h^{0}\left(\mathcal{L}^{-k}\right)=k$.
} 
define $I^{\prime}(k)$, which is not zero, and gives non-trivial information about the number of vacua 68, 10.

To summarise, in the case $N=1, N_{M 2}=0$ we find that M-theory on $X=\mathbb{B}_{8}$ gives a $U(1)$ effective gauge theory with half-integer Chern-Simons term $k=k_{0}+\frac{1}{2}$. The theory is parametrised by one real parameter - the gauge coupling constant. There is only one classical limit, corresponding to large $\operatorname{Vol}\left(\mathbf{S}^{4}\right)$. For all values of $k_{0} \in \mathbb{Z}$ this theory is expected to be infrared-free and to have only one massive vacuum.

One can consider various generalizations of this model corresponding to $N>1$ and/or $N_{M 2}>0$. Let us briefly comment on the models with $N>1$. In Type IIA theory they correspond to configurations of multiple D6-branes wrapped on the coassociative four-cycle $B=\mathbf{S}^{4}$ inside the $G 2$ space $M^{7}=\Lambda^{-} \mathbf{S}^{4}$. Now, the effective field theory on the D6-branes is $U(N)$ gauge theory with Chern-Simons coefficient congruent to $N / 2 \bmod \mathbb{Z}$ :

$$
L_{3 d}=\frac{1}{4 g^{2}} \int d^{3} x \operatorname{Tr}\left(\mathcal{F}_{\mu \nu} \mathcal{F}^{\mu \nu}\right)+\frac{i\left(k_{0}+\frac{N}{2}\right)}{4 \pi} \int_{\mathbb{R}^{3}} \operatorname{Tr}\left(\mathcal{A} \wedge d \mathcal{A}+\frac{2}{3} \mathcal{A} \wedge \mathcal{A} \wedge \mathcal{A}\right)+\text { fermions }
$$

Like in the $N=1$ case, the gauge coupling in this theory is related to the volume of the four-sphere, whereas $k_{0}$ is determined by the $G$-flux through the $\mathbf{S}^{4}, c f$. (6.65):

$$
\int_{\mathbf{S}^{4}}\left[\frac{G}{2 \pi}\right]=k_{0}+\frac{N}{2}
$$

In M-theory this non-abelian theory is expected to come from compactification on a singular $\operatorname{Spin}(7)$ manifold $X$, which is an $\mathbb{R}^{4} / \mathbb{Z}_{N}$ bundle over $\mathbf{S}^{4}$. The boundary of $X$ is a seven-manifold $Y=\mathbf{S}^{7} / \mathbb{Z}_{N}$. Since $\mathbb{Z}_{N}$ acts freely on $\mathbf{S}^{7}, Y$ is smooth, and we have:

$$
H^{4}(Y ; \mathbb{Z}) \cong \mathbb{Z}_{N}
$$

Therefore, the model is specified now by $\Phi_{\infty}$ and a (half)-integer number $k=k_{0}+\frac{N}{2}$ defined modulo $N$. At least for large values of $\Phi_{\infty}$, where the IR dynamics is dual to $\mathrm{AdS}_{4} \times Y$, the theory is expected to flow to a non-trivial $\mathcal{N}=1$ superconformal field theory.

For a given value of $k$, this effective theory (compactified on an extra $T^{2}$ ) is expected to have $I_{U(N)}^{\prime}(k)$ supersymmetric vacua, where:

$$
I_{U(N)}^{\prime}(k)=\frac{I^{\prime}(k) \cdot(k+N / 2-1) !}{N !(k-N / 2) !}
$$

In order to compute $I_{U(N)}^{\prime}(k)$ one can think of the $U(N)$ gauge group as a product $U(1) \times S U(N) / \mathbb{Z}_{N}, c f$. [68, 10]. Then, $I_{U(N)}^{\prime}(k)$ is a product of the number of ground 
states in the $U(1)$ gauge theory and the number of vacua in the $S U(N)$ gauge theory, divided by $N$. We remind the reader that in $\mathcal{N}=1$ three-dimensional gauge theory with gauge group $S U(N)$ the supersymmetric index is [9]:

$$
I_{S U(N)}=\frac{(k+N / 2-1) !}{(N-1) !(k-N / 2) !}
$$

where $|k|>N / 2$. By definition, $I_{S U(N)}$ gives the number of bosonic ground states minus the number of fermionic ground states. However, it was argued in [9] that all ground states in three-dimensional $S U(N)$ super-Yang-Mills theory are bosonic, so that $I_{S U(N)}$ gives the actual number of ground states. On the other hand, the supersymmetric index in $U(N)$ gauge theory is zero due to the fermionic zero mode in the "central" $U(1)$, as we remarked ealier.

In [9], by analysing the supersymmetric index it was shown that supersymmetry is unbroken in this quantum theory for $k \geq N / 2$, whereas strong evidence was given to support the conjecture that supersymmetry is dynamically broken for $k<N / 2$. It would be interesting to study this problem in eleven-dimensional supergravity, constructing an explicit solution with non-zero $G$-flux, and to understand the relation of such a solution to the octonionic superstring soliton constructed in [69].

\subsection{M-Theory on $\operatorname{Spin}^{c}$ Bundles over $\mathbb{C} P^{2}$}

The dynamics of the models based on the total space of $\left(\mathbb{Z}_{N}\right.$ quotients of $) \operatorname{spin}^{c}$ bundles over $\mathbb{C P}^{2}$ is more interesting and subtle, even in the simple case $N=1$. In fact, instead of a single parameter $N$ we have two integer numbers, $k$ and $l$, which parametrise different types of complete $\operatorname{Spin}(7)$ metrics on $X$. The corresponding manifolds are asymptotically locally conical with principal orbits

$$
Y=S U(3) / T_{k, l} \cong S U(3) / U(1)
$$

Here, following our notations in section $4, k$ and $l$ parametrise different $U(1)$ actions on $S U(3)$. For example, for $k=-l=-1$ the explicit metric was constructed in (4.54), and describes a complete metric of $\operatorname{Spin}(7)$ holonomy on $\mathcal{Q}$ :

$$
\begin{aligned}
d s^{2}= & \frac{(r-a)(r+a)}{(r-2 a)(r+2 a)} d r^{2}+\frac{9 a^{2}}{8} \frac{(r-2 a)(r+2 a)}{(r-a)(r+a)} \lambda^{2}+(r+a)(r-2 a)\left(\sigma_{1}^{2}+\sigma_{2}^{2}\right)+ \\
& +r^{2}\left(\Sigma_{1}^{2}+\Sigma_{2}^{2}\right)+(r-a)(r+2 a)\left(\nu_{1}^{2}+\nu_{2}^{2}\right)
\end{aligned}
$$

In general, a $\operatorname{Spin}(7)$ metric on a $\left(\mathbb{Z}_{N}\right.$ quotient of a) $\operatorname{spin}^{c}$ bundle over $\mathbb{C} \mathbf{P}^{2}$ can be written in the form (4.45):

$$
d s^{2}=d t^{2}+f^{2} \lambda^{2}+a^{2}\left(\sigma_{1}^{2}+\sigma_{2}^{2}\right)+b^{2}\left(\Sigma_{1}^{2}+\Sigma_{2}^{2}\right)+c^{2}\left(\nu_{1}^{2}+\nu_{2}^{2}\right)
$$


where $a, b, c$, and $f$ are certain functions of the radial variable $t$. Other examples of $\operatorname{Spin}(7)$ metrics on such $\mathbb{R}^{4} / \mathbb{Z}_{N}$ bundles over $\mathbb{C P}^{2}$ can be found in [24] and also in the recent paper [16]. Indeed, in the latter reference, explicit asymptotically locally conical metrics were found for all values of $k$ and $l$, generalising our solution (6.76). They also found evidence for the existence of asymptotically conical versions of these solutions, one of which is given by the AC metric on $T^{*} \mathbb{C} \mathbf{P}^{2} / \mathbb{Z}_{2}(2.6)$. When the size of the Cayley $\mathbb{C P}^{2}$ goes to zero, these manifolds develop a conical singularity, and our goal in this section will be to understand the behaviour of M-theory near such a singularity. For these purposes, we can often take $X$ to be (asymptotically) a cone over $Y$.

Following [2], let us study the global symmetries of these models. The classical symmetries of the three-dimensional theory correspond to geometrical symmetries of $Y$ and gauge symmetries of the $C$-field, classified by $H^{2}(Y ; U(1))$. The symmetries which extend to the entire eight-manifold $X$ can be identified with the symmetries of the quantum theory.

First, let us describe the geometrical symmetries. Since $Y$ can be represented as a quotient space (6.75), it is invariant under the left action of $S U(3)$. Moreover, since the maximal torus of $S U(3)$ is two-dimensional, there is a $U(1)_{K} \subset S U(3)$, such that $U(1)_{K}$ centralises $T_{k, l}$. Therefore, $U(1)_{K}$ is also a symmetry of this model.

Let us now describe the symmetries associated with the gauge transformations of the $C$-field. The spectral sequence for the fibration $U(1) \hookrightarrow S U(3) \rightarrow Y$ gives:

$$
H^{2}(Y ; U(1))=H^{1}(U(1) ; U(1))=U(1)_{C}
$$

Therefore, $H^{2}(Y ; U(1))=U(1)_{C}$ is a classical symmetry of the three-dimensional $\mathcal{N}=$ 1 effective field theory. In fact this symmetry is unbroken in the quantum theory since $X$ is contractible to $\mathbb{C} \mathbf{P}^{2}$ and $H^{2}\left(\mathbb{C P}^{2} ; U(1)\right)=U(1)_{C}$.

To summarise, the effective three-dimensional $\mathcal{N}=1$ gauge theory has the following classical symmetry group:

$$
S U(3) \times U(1)_{K} \times U(1)_{C}
$$

In the case $k=l$ we have an additional discrete $\Sigma_{2}$ symmetry.

Let us now examine the equivalent D6-brane description of the same model, discussed in section 3. Namely, we found that there is a semi-free $U(1)$ action on $X=\mathcal{Q}$, such that $X / U(1) \cong \mathbb{R}^{7}$ and the fixed point set is a union of two disconnected four-manifolds:

$$
L=\mathbb{R}^{4} \cup \mathbb{R}^{2} \times \mathbf{S}^{2} \subset \mathbb{R}^{7}
$$

Therefore, M-theory on the $\operatorname{Spin}(7)$ space $X$ can equivalently be described as a D6brane on $\mathbb{R}^{3} \times L$ in Type IIA string theory. The point in the moduli space where $X$ develops a singularity corresponds in this language to the singular configuration 
of two intersecting D6-branes on $L=\mathbb{R}^{4} \cup \mathbb{R}^{4}$. Note that at this point a string stretched between two connected components of the D6-brane world-volume is lifted to a membrane wrapped on a topologically non-trivial two-cycle in M-theory on $X$.

As in the earlier sections, we also find very useful the Type IIA description of the same system in terms of D6-branes wrapped on the coassociative four-cycle $B=\mathbb{C P}^{2}$ inside the G2-holonomy manifold

$$
M^{7}=\Lambda^{-} \mathbb{C P}^{2}
$$

The number of D6-branes is determined by the topology of $X$. Namely, we have:

$$
N=|k|,|l|, \text { or }|k+l|
$$

depending on how the Aloff-Wallach space $N_{k, l}$ is resolved. In this Type IIA description one can easily see the spectrum of massless modes. It suffices to count only bosonic fields since fermionic modes will complete the $\mathcal{N}=1$ supermultiplets. The only bosonic field on the D6-branes is a $U(N)$ gauge field, which, after reduction on $B$, gives a $U(N)$ gauge field in three dimensions. Indeed, since $B$ is simply-connected and $b_{2}^{-}(B)=0$ there are no extra bosonic fields in three dimensions.

However, there might be some bulk modes. Specifically, one might have a $U(1)$ gauge field $\mathcal{A}_{0}$ from the decomposition of the 3 -form field $C$ along the generator $\omega_{0}^{(2)}$ of $H^{2}\left(M^{7} ; \mathbb{Z}\right)$. Furthermore, there could also be a scalar field from the deformation of the $\operatorname{Spin}(7)$ metric on $X$. However, these bulk fields are not dynamical since the corresponding modes are not $L^{2}$ normalisable [2]. For example, the $L^{2}$ norm, $|\delta g|^{2}$, of the deformation of the $\operatorname{Spin}(7)$ metric on $\mathcal{Q}$ corresponding to a change in the parameter $a$ is divergent, just as in the $\mathbb{B}_{8}$ case.

From this description we can also derive the effective action of the three-dimensional theory. For simplicity, let us focus on the case $N=1$. Then, the effective $\mathcal{N}=1$ theory is a $U(1)$ gauge theory with Chern-Simons coupling of the form (6.41). The model can be viewed as a limit $g_{1} \rightarrow 0$ of the $U(1)_{0} \times U(1)_{1}$ gauge theory with the following Lagrangian:

$L_{3 d}=\int d^{3} x\left(\frac{1}{4 g_{0}^{2}} \mathcal{F}_{0}^{2}+\frac{1}{4 g_{1}^{2}} \mathcal{F}_{1}^{2}\right)-\int\left(\frac{i k_{00}}{4 \pi} \mathcal{A}_{0} \wedge d \mathcal{A}_{0}+\frac{i k_{11}}{4 \pi} \mathcal{A}_{1} \wedge d \mathcal{A}_{1}+\frac{i k_{01}}{4 \pi} \mathcal{A}_{0} \wedge d \mathcal{A}_{1}\right)$

The "off-diagonal" Chern-Simons coefficient $k_{01}$ is proportional to the flux of the $U(1)$ gauge field on the D6-brane through the basic two-cycle $\mathbf{S}^{2} \in H_{2}\left(\mathbb{C} \mathbf{P}^{2} ; \mathbb{Z}\right), c f$. (6.25):

$$
k_{01}=\int_{\mathbf{S}^{2}} \frac{\mathcal{F}}{2 \pi}
$$


As we discussed in section 3, following [38], this flux is half-integer and, in particular, cannot be zero. Namely, it is convenient to introduce an integer number $\tilde{k}_{01} \in \mathbb{Z}$, such that:

$$
k_{01}=\tilde{k}_{01}+\frac{1}{2}
$$

This D-brane description suggests that this flux is also related to the choice of $\operatorname{spin}^{c}$ structure on $\mathbb{C P}^{2}$, which in turn is related to the flux of the RR two-form field strength $G_{2}$.

As in gravity duals of gauge theories with larger amount of supersymmetry [70, 71], the gauge coupling constant $g_{0}$ in the Lagrangian (6.83) is proportional to the D6-brane tension:

$$
\frac{1}{g^{2}} \sim \int_{\mathbb{C P}^{2}} d^{4} x \sqrt{-\operatorname{det}\left(g_{m n}+2 \pi \alpha^{\prime} \mathcal{F}_{m n}\right)}
$$

Therefore, in the limit $\operatorname{Vol}\left(\mathbb{C P}^{2}\right) \rightarrow 0$ the value of the coupling constant remains finite (determined by the $\mathcal{F}$-flux through $\mathbf{S}^{2} \subset \mathbb{C P}^{2}$ ):

$$
\frac{1}{g^{2}} \sim\left(\tilde{k}_{01}+\frac{1}{2}\right)^{2}
$$

This suggests that, even if the manifold $X$ develops a geometrical singularity, the dynamics of M-theory on $X$ is still non-singular.

The Chern-Simons couplings $k_{00}$ and $k_{11}$ are determined by the background $G$-flux. Specifically, $k_{11}$ is given by (6.41), whereas $k_{00}$ depends also on the $G_{2}$-flux and $\mathcal{F}$-flux through the $\mathbf{S}^{2} \in H_{2}\left(\mathbb{C} \mathbf{P}^{2} ; \mathbb{Z}\right)$ :

$$
k_{00}=k_{0}+\frac{1}{2}+\left(\tilde{k}_{01}+\frac{1}{2}\right)^{2}-\left(\tilde{k}_{01}+\frac{1}{2}\right) \cdot \int_{\mathbf{S}^{2}} \frac{G_{2}}{2 \pi}
$$

Here we follow the normalisation of (6.30):

$$
\int_{\mathbb{C P}^{2}}\left[\frac{G}{2 \pi}\right]=k_{0}+\frac{1}{2}
$$

In order to classify the models and, in particular, to compute the restriction of the $C$-field to $Y=\partial X$, we need to know $H^{4}(Y ; \mathbb{Z})$. For a general space $X_{k, l}$ over $\mathbb{C P}^{2}$ this group is:

$$
H^{4}(Y ; \mathbb{Z})=\mathbb{Z}_{r}, \quad \text { where } \quad r=\left|k^{2}+l^{2}+k l\right|
$$

In particular, if we restrict ourselves to $N=1$ (corresponding to the set of $\operatorname{spin}^{c}$ bundles over $\mathbb{C P}^{2}$ ), we end up with only one parameter, $p$, which labels the $\operatorname{spin}^{c}$ structure on $\mathbb{C P}^{2}$. The corresponding Aloff-Wallach spaces look like $Y=N_{1, p}$, and $H^{4}(Y ; \mathbb{Z}) \cong \mathbb{Z}_{r(p)}$ where

$$
r(p)=1+p+p^{2}
$$


In particular, in the case $p=0$ we get the $\operatorname{Spin}(7)$ manifold (4.54), while in the case $p=1$ we get the cotangent bundle of $\mathbb{C P}^{2}$ with $H^{4}(Y ; \mathbb{Z}) \cong \mathbb{Z}_{3}$. The vacua in the latter model which preserve $\mathcal{N}=3$ supersymmetry in three dimensions were studied in [37. This model is related to the corresponding Spin(7) manifold in question by means of orientifold projection; see appendix B for details. For general $r(p)$ we find that different models are classified by an integer $k_{0} \bmod r$, and also by the value of $\Phi_{\infty}$ :

$$
\Phi_{\infty}+\chi(X)=\frac{1}{2 r}\left(k_{0}+\frac{r}{2}\right)^{2}
$$

Here we have used the fact that the self-intersection number of $B=\mathbb{C P}^{2}$ is equal to $r$, and also the following convention for the integral of the $G$-flux, $c f$. (6.89):

$$
\int_{\mathbb{C} \mathbf{P}^{2}}\left[\frac{G}{2 \pi}\right]=k_{0}+\frac{r}{2}
$$

A consistency check on the formula (6.92) is that $\Phi_{\infty}$ shifts by an integer when $k_{0}$ is shifted by a multiple of $r$.

All models with $p=0$ have a unique massive vacuum. Just like the model based on $X=\mathbb{B}_{8}$ that we discussed in the previous subsection, they are uniquely specified by the value of $\Phi_{\infty}$.

The same is true for most of the models with $p>0$, except for those where $\Phi_{\infty}$ is related to $r(p)$ as follows:

$$
\Phi_{\infty}=\frac{r}{8}-\chi(X)=\frac{1+p+p^{2}}{8}-\chi(X)
$$

These models have two vacua at $k_{0}=0$ and $k_{0}=-r(p)$.

The value of $\chi(X)$ in the equation (6.92) can again be computed by comparing this model to Type IIA theory with a D6-brane wrapped on the coassociative 4-cycle $B=\mathbb{C P}^{2}$. However, in this case the calculation is more subtle, since there is a nontrivial flux (6.84) on the brane, and one has to use the more general formula (6.11). Specifically, from the relation (6.11) we get:

$$
\frac{\chi(X)}{24}=\frac{1}{48}\left(p_{1}\left(N_{\mathbb{C P}^{2}}\right)-p_{1}\left(T_{\mathbb{C P}^{2}}\right)\right)+\frac{1}{2}\left(\tilde{k}_{01}+\frac{1}{2}\right)^{2}
$$

The first two terms in this expression are computed in Appendix A. Their total contribution to $\chi(X)$ is $-1 / 8$. Note that the second terms looks very similar to the right-hand side of (6.92) which suggests a relation between the $F$-flux on the D6brane, parametrised by $k_{01}$, with the $G$-flux in M-theory on $X$, parametrised by $k_{0}$. This identification agrees with other arguments we found in the present section and in section 3 . 
For large values of $\Phi_{\infty}$ we expect that the model can be described by M-theory on $\mathrm{AdS}_{4} \times Y$ where $Y=S U(3) / U(1)$ is the Aloff-Wallach space discussed in section 4 . Therefore, at least for large $\Phi_{\infty}$ we expect our models to flow to a non-trivial $\mathcal{N}=1$ superconformal fixed point in the infra-red.

\section{Acknowledgments}

We wish to thank K. Costello, G. Gibbons, C. Herzog, J. Maldacena, N. Nekrasov, C. Núñez, C. Pope, E. Rabinovici, S. Schafer-Nameki, A. Strominger, C. Vafa, and E. Witten for useful discussions. This research was partially conducted during the period S.G. served as a Clay Mathematics Institute Long-Term Prize Fellow. The work of S.G. is also supported in part by grant RFBR No. 01-02-17488, and the Russian President's grant No. 00-15-99296.

\section{A Computation of Pontryagin classes}

In this Appendix, we compute the first Pontryagin class of the bundle of anti-self-dual two-forms over $B$, where $B$ is either $\mathbf{S}^{4}$ or $\mathbb{C} \mathbf{P}^{2}$. This is a fairly standard calculation involving Chern classes. One may find an explanation of most of the tools used here in 43 .

The key point in the calculation is to note (see, for example, [23]) that the complexification of $\Lambda^{-} \equiv \Lambda^{-} B$ satisfies

$$
\Lambda_{\mathbb{C}}^{-} \equiv \Lambda^{-} \otimes \mathbb{C} \cong S^{2} \Sigma_{-}
$$

where $\Sigma_{-}$is the spin bundle of $B$, and $S^{2} E$ denotes the second symmetric power product of the (complex) vector bundle $\mathrm{E}$. This is just like the exterior power $\Lambda$, but instead we take the symmetrised tensor product, rather than the antisymmetrised product. Counting dimensions, we see that, according to (A.1) $\Lambda_{\mathbb{C}}^{-}$is a rank three complex vector bundle over $B$, which is indeed correct.

By definition, we have

$$
p_{1}\left(\Lambda^{-}\right) \equiv-c_{2}\left(\Lambda_{\mathbb{C}}^{-}\right)=-c_{2}\left(S^{2} \Sigma_{-}\right)
$$

We therefore need to compute the Chern class $c\left(S^{2} E\right)$ for $E$ a complex rank two vector bundle. This is a fairly standard calculation. It is easy to verify that

$$
c\left(S^{2} E\right)=\prod_{1 \leq i_{1} \leq i_{2} \leq 2}\left(1+x_{i_{1}}+x_{i_{2}}\right)
$$


where $x_{1}, x_{2}$ are the first Chern classes of the line bundle into which $E$ splits when pulled back to the splitting manifold i.e.

$$
c(E)=\prod_{i=1}^{2}\left(1+x_{i}\right)
$$

This gives

$$
c\left(S^{2} E\right)=\left(1+2 x_{1}\right)\left(1+x_{1}+x_{2}\right)\left(1+2 x_{2}\right)
$$

Since this is symmetric in $x_{1}, x_{2}$, we must be able to write it in terms of Chern classes. One obtains

$$
c\left(S^{2} E\right)=1+3 c_{1}+2 c_{1}^{2}+4 c_{2}+4 c_{1} c_{2}
$$

and so we pick out

$$
c_{2}\left(S^{2} E\right)=2 c_{1}^{2}+4 c_{2}
$$

Thus, putting everything together, we get

$$
p_{1}\left(\Lambda^{-}\right)=-\left(2 c_{1}^{2}+4 c_{2}\right)
$$

where $c=c\left(\Sigma_{-}\right)$are the Chern classes of $\Sigma_{-}$.

We may apply this directly to $\mathbf{S}^{4}$. $c_{1}$ is obviously zero, and

$$
c_{2}\left(\Sigma_{-}\right)=e\left(\Sigma_{-, \mathbb{R}}\right)=u
$$

where $u$ generates $H^{4}\left(\mathbf{S}^{4} ; \mathbb{Z}\right) \cong \mathbb{Z}$, and so

$$
p_{1}\left(\Lambda^{-} \mathbf{S}^{4}\right)=-4 u
$$

For $\mathbb{C P}^{2}$, we have to work a little bit harder, since $\Sigma_{-}$doesn't exist. However, we can pick a $\operatorname{spin}^{c}$ bundle

$$
\mathbb{V}_{-}(L) \equiv \Sigma_{-} \otimes L^{1 / 2}
$$

with complex line bundle $L$ which has first Chern class $c_{1}(L)=c_{1}\left(\mathbb{V}_{-}(L)\right)=n x$ where $n$ is an odd integer and $x$ generates the cohomology ring of $\mathbb{C P}^{2}$. Then the symmetric product bundle $S^{2} \Sigma_{-}$does exist, and satisfies 


$$
S^{2} \Sigma_{-} \cong L^{-1} \otimes S^{2} \mathbb{V}_{-}(L)
$$

We first set $F=\mathbb{V}_{-}(L)$ so that $c_{1}(F)=n x$ and $c_{2}(F)=x^{2}+\frac{c_{1}(L)^{2}-x^{2}}{4}$. Thus we have

$$
\begin{aligned}
& c_{1}\left(S^{2} F\right)=3 c_{1}(F)=3 n x \\
& c_{2}\left(S^{2} F\right)=2 c_{1}(F)^{2}+4 c_{2}(F)=2 n^{2} x^{2}+4 x^{2}+\left(n^{2}-1\right) x^{2}=3\left(n^{2}+1\right) x^{2} \\
& c_{3}\left(S^{2} F\right)=4 c_{1}(F) c_{2}(F)=4 n x\left(1+\frac{n^{2}-1}{4}\right) x^{2}
\end{aligned}
$$

Then

$$
\begin{gathered}
c\left(S^{2} \Sigma_{-}\right)=c\left(L^{-1} \otimes S^{2} \mathbb{V}_{-}(L)\right) \\
\quad=\sum_{i=0}^{3} c_{i}\left(S^{2} F\right)(1-n x)^{3-i}
\end{gathered}
$$

After some algebra, one eventually finds

$$
c_{2}\left(S^{2} \Sigma_{-}\right)=3 n^{2} x^{2}-6 n^{2} x^{2}+3 n^{2} x^{2}+3 x^{2}=3 x^{2}
$$

The fact that $n$ drops out here is of course a good check on the calculation, since the "spin bundle" $\Sigma_{-}$doesn't depend on $n$. So, we get

$$
p_{1}\left(\Lambda^{-} \mathbb{C} \mathbf{P}^{2}\right)=-c_{2}\left(S^{2} \Sigma_{-}\right)=-3 x^{2}
$$

\section{B Joyce Construction of Spin(7) Manifolds}

In this appendix we explain how a new manifold of $\operatorname{Spin}(7)$ holonomy can be constructed from the cotangent bundle of $\mathbb{C P}^{2}$, with the Ricci-flat Calabi metric [25]. Our goal here will be to find a suitable anti-holomorphic involution $\tau$ on $T^{*} \mathbb{C P}^{2}$, such that $\tau$ does not have fixed points. Then, according to [72], the quotient space $X=T^{*} \mathbb{C P}^{2} / \tau$ is a manifold of $\operatorname{Spin}(7)$ holonomy.

Let us start with the construction of $T^{*} \mathbb{C} P^{2}$ itself. We can describe this manifold in terms of two sets of complex variables $B_{i}$ and $C_{i}$ which, respectively, have charges +1 and -1 under the action of the $U(1)$ symmetry group [37. Both $B_{i}$ and $C_{i}$ transform as the 3 of the $S U(3)$ global symmetry group that will also be a symmetry of the 
quotient space $X$. Furthermore, $T^{*} \mathbb{C P}^{2}$ is invariant under $S U(2)$, under which $\left(B_{i}, \bar{C}_{i}\right)$ transform as a doublet for every $i=1,2,3$.

In these variables the space in question is described by one real and one complex equation:

$$
\sum_{i}\left|B_{i}\right|^{2}-\left|C_{i}\right|^{2}=d_{r}
$$

and

$$
\sum_{i} B_{i} C_{i}=d_{c}
$$

where $d_{r} \in \mathbb{R}$ and $d_{c} \in \mathbb{C}$. After we divide by the action of $U(1)$, we obtain an eight-dimensional manifold, $T^{*} \mathbb{C P}^{2}$, asymptotic to a cone over $N_{1,1}=S U(3) / U(1)$.

Now, consider the following anti-holomorphic involution:

$$
\tau: B_{i} \rightarrow \bar{C}_{i}
$$

which manifestly preserves the $S U(3)$ symmetry group.

The real equation $(\mathbb{B} .1)$ is compatible with this involution only if $d_{r}=0$, so that we can write it as:

$$
\sum_{i}\left|B_{i}\right|^{2}=\sum\left|C_{i}\right|^{2}
$$

On the left-hand side of the other equation (B.2) the involution $\tau$ acts by complex conjugation. Hence, $d_{c}$ must be real; we denote it simply $d$. Then, from the equation $(\mathrm{B} .2)$, we find:

$$
\sum_{i} B_{i} C_{i}=d
$$

To summarise, $X=T^{*} \mathbb{C} P^{2} / \tau$ is locally described by two equations (one real equation $(\mathbb{B . 4})$ and one complex equation $(\mathbb{B} .5)$ ), divided by the action of $U(1)$ and $\tau$. According to [72], the quotient space $X$ is a (singular) $\operatorname{Spin}(7)$ manifold. We wish to focus on the simple case where the involution $\tau$ has no fixed point 29 .

The fixed points of the involution are at $B_{i}=\bar{C}_{i}$. Substituting this into (B.5) we get:

$$
\sum_{i}\left|B_{i}\right|^{2}=d
$$

So, the set of fixed points is a copy of $\mathbb{C P}^{2}$ for $d>0$, and an empty set for $d<0$. We wish to focus on the second possibility, and examine the topology of the resulting space. It is convenient to introduce new complex variables $M_{i}$ and $N_{i}$, such that:

$$
B_{i}=M_{i}+N_{i}, \quad C_{i}=\bar{M}_{i}-\bar{N}_{i}
$$

\footnotetext{
${ }^{29}$ In general, if $\tau$ has isolated fixed points of a suitable kind, one may resolve them following the construction of Joyce [21].
} 
The new variables $M_{i}$ and $N_{i}$ both have charge +1 under the action of $U(1)$ and transform in the following way under the action of $\tau$ :

$$
\begin{aligned}
M_{i} & \rightarrow M_{i} \\
N_{i} & \rightarrow-N_{i}
\end{aligned}
$$

We can rewrite equation (B.4) as:

$$
\sum_{i}\left(M_{i} \bar{N}_{i}+\bar{N}_{i} M_{i}\right)=0
$$

On the other hand, from (B.5) we get two equations, corresponding to the real and imaginary parts, respectively:

$$
\begin{aligned}
& \sum_{i}\left(\left|M_{i}\right|^{2}-\left|N_{i}\right|^{2}\right)=d, \\
& \sum_{i}\left(M_{i} \bar{N}_{i}-\bar{N}_{i} M_{i}\right)=0
\end{aligned}
$$

From equations $(\overline{B .9})$ and $(\overline{B .11})$ we find:

$$
\sum M_{i} \bar{N}_{i}=0
$$

which is very simlar to equation $(\overline{B .5})$. Together with $B .10$ it describes the space with the expected topology, cf. [37]. Indeed, since $d$ is assumed to be negative, one can introduce a new variable:

$$
Z_{i}=\frac{N_{i}}{\sqrt{-d+\sum_{i}\left|M_{i}\right|^{2}}}
$$

which takes values in $\mathbf{S}^{5}$. After dividing by the $U(1)$ symmetry we get a copy of $\mathbb{C P}^{2}$. In principle, one should also divide out by the action of $\tau$, which acts as $Z_{i} \rightarrow-Z_{i}$. However, on the $\mathbf{S}^{5}$ this transformation is equivalent to a $U(1)$ gauge transformation. Finally, from equation $(\mathbb{B . 1 2})$ it follows that $X$ is an $\mathbb{R}^{4}$ bundle over $\mathbb{C P}^{2}$.

One would expect that the new manifold $\mathbb{C}_{8}$ of $\operatorname{Spin}(7)$ holonomy found recently in [16] may also be constructed from an $\mathcal{O}(-4)$ bundle over $\mathbb{C P}^{3}$, after dividing by a suitable anti-holomorphic involution, as in [72.

\section{References}

[1] M. Atiyah, J. Maldacena and C. Vafa, "An M-theory flop as a large N duality," hep-th/0011256. 
[2] M.F. Atiyah, E. Witten, "M-Theory Dynamics On A Manifold of $G_{2}$ Holonomy," hep-th/0107177.

[3] E. Witten, "Is Supersymmetry Really Broken?", hep-th/9409111.

[4] E. Witten, "Strong Coupling And The Cosmological Constant," hep-th/9506101.

[5] M. Aganagic and C. Vafa, "Mirror Symmetry and a G(2) Flop," hep-th/0105225.

[6] I. Affleck, J. Harvey, E. Witten, "Instantons and (super)symmetry breaking in (2+1)-dimensions," Nucl. Phys. 206 (1982) 413.

[7] M. Gremm and E. Katz, "Mirror Symmetry of N=1 QED in Three Dimensions," hep-th/9906020.

[8] M. Berkooz, A. Kapustin, M. Strassler, as ref.[14] in [7]

[9] E. Witten, "Supersymmetric Index Of Three-Dimensional Gauge Theory", hep-th/ 9903005.

[10] J. Maldacena, H. Nastase, "The Supergravity Dual of a Gauge Theory with Dynamical Supersymmetry Breaking," hep-th/0105049.

[11] R. Bryant, S. Salamon, "On the Construction of some Complete Metrics with Exceptional Holonomy", Duke Math. J. 58 (1989) 829.

G. W. Gibbons, D. N. Page, C. N. Pope, "Einstein Metrics on $S^{3}, \mathbb{R}^{3}$ and $\mathbb{R}^{4}$ Bundles" Commun.Math.Phys 127 (1990) 529-553.

[12] J. Gomis, "D-Branes, Holonomy and M-Theory", hep-th/0103115.

[13] R. Harvey and H.B. Lawson, Jr., "Calibrated geometries", Acta Math. 148 (1982) 47.

[14] K. Becker, M. Becker, D.R. Morrison, H. Ooguri, Y. Oz, Z. Yin, "Supersymmetric Cycles in Exceptional Holonomy Manifolds and Calabi-Yau 4-Folds", Nucl.Phys. B480 (1996) 225.

[15] M. Cvetic, G.W. Gibbons, H. Lu, C.N. Pope, "New Complete Non-compact Spin(7) Manifolds," hep-th/0103155.

[16] M. Cvetic, G.W. Gibbons, H. Lu, C.N. Pope, "Cohomogeneity One Manifolds of Spin(7) and G2 Holonomy" hep-th/0108245.

[17] N. Hitchin, "Stable forms and special metrics," math.DG/0107101. 
[18] E. Witten, "On Flux Quantisation in M-Theory and the Effective Action" J.Geom.Phys 22 (1997) 1-13, hep-th/9609122.

[19] H. Kanno, Y. Yasui, "On $\operatorname{Spin}(7)$ holonomy metric based on $S U(3) / U(1)$ ", hep-th/0108226.

[20] in progress.

[21] D. Joyce, "Compact Manifolds with Special Holonomy", Oxford University Press, 2000.

[22] R. C. McLean, "Deformations of Calibrated Submanifolds", PhD Thesis.

[23] A. Besse, "Einstein Manifolds", Springer-Verlag, 1987.

[24] M. Cvetic, G. W. Gibbons, H. Lu, C. N. Pope, "Hyper-Kähler Calabi Metrics, $L^{2}$ Harmonic Forms, Resolved M2-branes, and $A d S_{4} / C F T_{3}$ Correspondence", hep-th/0102185.

[25] E. Calabi, "Métriques Kahlériennes et fibrés holomorphes", Ann. Scient. École Norm. Sup. 12 (1979) 269

[26] G. Bredon, "Exotic actions on spheres," Proceedings of the conference on transformation groups, New Orleans (1967) 47.

[27] D. Montgomery, L. Zippin, "Topological transformation groups," New York: Interscience, 1955.

[28] J. Levine, "Semi-Free Circle Actions on Spheres," Invent. Math. 22 (1973) 161.

[29] G.W. Gibbons and G. Papadopoulos, "Calibrations and Intersecting Branes", Commun. Math. Phys. 202 (1999) 593.

[30] J.P. Gauntlett, N.D. Lambert, P.C. West, "Branes and Calibrated Geometries", Commun.Math.Phys. 202 (1999) 571.

[31] B. S. Acharya, J. M. Figueroa-O'Farrill, B. Spence, "Branes at angles and calibrated geometry", JHEP 9804 (1998) 012.

[32] J.M. Figueroa-O'Farrill, "Intersecting brane geometries", hep-th/9806040.

[33] G. Papadopoulos and A. Teschendorff, "Grassmannians, Calibrations and FiveBrane Intersections", hep-th/9811034. 
[34] J. Gutowski, G. Papadopoulos, P. K. Townsend, "Supersymmetry and Generalized Calibrations", hep-th/9905156.

[35] P.K. Townsend, "Brane Surgery," hep-th/9609217.

[36] M. B. Green, J. A. Havrey, G. Moore, "I-Brane Inflow and Anomalous Couplings on D-Branes", Class. Quant. Grav. 14 (1997) 47, hep-th/9605033.

[37] S. Gukov, C. Vafa, E. Witten, "CFT's From Calabi-Yau Four-folds," Nucl.Phys. B584 (2000) 69.

[38] D. S. Freed, E. Witten, "Anomalies in String Theory with D-Branes", hep-th/9907189.

[39] J. de Boer, R. Dijkgraaf, K. Hori, A. Keurentjes, J. Morgan, D.R. Morrison, and S. Sethi, "Triples, Fluxes, and Strings," hep-th/0103170.

[40] E. Witten, "Topological Tools in 10-Dimensional Physics", Int.Journ.Mod.Phys A Vol.1 No. 1 (1986) 39-64.

[41] R. Schults, "Exotic Spheres Admitting Circle Actions with Codimension Four Stationary Sets," Contemp. Math. 19 (1983) 339.

[42] S. Aloff, N. R. Wallach, "An Infinite Family of Distinct 7-Manifolds Admitting Positively Curved Riemannian Structures", Bull. Am. Math. Soc. 81 No.1 (1975) 93.

Wang, Y. McKenzie, "Some Examples of Homogeneous Einstein Manifolds in Dimension Seven", Duke Math. J. 49 (1982) 23

D. N. Page, C. N. Pope, "New Squashed Solutions to $d=11$ Supergravity", Phys. Lett. 147B (1985) 55

M. J. Duff, B. E. W. Nilsson, C. N. Pope, "Kaluza-Klein Supergravity", Phys. Rep. 130 (1986) 1.

[43] R. Bott, L. W. Tu, "Differential Forms in Algebraic Topology", Springer-Verlag, 1982.

[44] J. W. Milnor, J. D. Stasheff, "Characteristic Classes", Annals of Mathematics Studies No. 76, Princeton University Press.

[45] A. Brandhuber, J. Gomis, S. S. Gubser, S. Gukov, "Gauge Theory at Large N and New $G_{2}$ Holonomy Metrics", hep-th/0106034. 
[46] R. L. Bryant, E. Sharpe, "D-Branes and Spin ${ }^{c}$ Structures", hep-th/9812084.

[47] E. Witten, "D-Branes and K-Theory" JHEP 9812 (1998) 019, hep-th/9810188.

[48] R. Hernández, "Branes Wrapped on Coassociative Cycles", hep-th/0106055.

[49] D.-E. Diaconescu, G. Moore, E. Witten, "E8 Gauge Theory, and a Derivation of K-Theory from M-Theory", hep-th/0005090.

[50] G. Moore, E. Witten, "Self-Duality, Ramond-Ramond Fields, and K-Theory" JHEP 0005 (2000) 032, hep-th/9912279.

[51] G. Papadopoulos, P.K. Townsend, "Compactification of D=11 supergravity on spaces of exceptional holonomy," Phys.Lett. B357 (1995) 300.

[52] S. Sethi, C. Vafa, and E. Witten, "Constraints on Low-Dimensional String Compactifications", Nucl. Phys. B480 (1996) 213.

[53] R. Minasian, G. Moore, "K-theory and Ramond-Ramond charge", J.High Energy Phys. 9711 (1997) 002.

[54] Y.-K. E. Cheung, Z. Yin, "Anomalies, Branes, and Currents", Nucl.Phys. B517 (1998) 69.

[55] H. B. Lawson, M.-L. Michelsohn, "Spin Geometry", Princeton University Press, 1989

[56] K. Becker, "A Note on Compactifications on Spin(7)-Holonomy Manifolds", hep-th/0011114.

[57] D. Montgomery, C.T. Yang, "Differentiable actions on homotopy seven-spheres: I," Trans. AMS 122 (1966) 480.

[58] M. Bershadsky, V. Sadov, and C. Vafa, "D-Branes and Topological Field Theories," hep-th/9511222.

[59] C. Vafa, E. Witten, "A Strong Coupling Test Of S-Duality," hep-th/9408074.

[60] S. Deser, R. Jackiw, and S. Templeton, Ann. Phys. 140 (1982) 372.

[61] G. Amelino-Camelia, I.I. Kogan, and R.S. Szabo, "Conformal Dimensions from Topologically Massive Quantum Field Theory," Nucl. Phys. B480 (1996) 413, hep-th/9607037. 
[62] H.-C. Kao, Kimyeong Lee, and Taelin Lee, "The Chern-Simons Coefficient In Supersymmetric Yang-Mills Chern-Simons Theories," hep-th/9506170.

[63] L. Alvarez-Gaumé and E. Witten, Nucl.Phys. B234 (1983) 269.

[64] N. Redlich, "Parity Violation and Gauge Noninvariance of the Effective Gauge Field Action in Three Dimensions," Phys. Rev. D29 (1984) 2366.

[65] E. Witten, "Five-Brane effective action in M theory", J.Geom.Phys. 22 (1997) 103, hep-th/9610234.

[66] S. Gukov, "Solitons, Superpotentials and Calibrations", hep-th/9911011.

[67] B.S. Acharya, X. de l'Ossa, S. Gukov, to appear.

[68] B. Acharya, C. Vafa, "On Domain Walls of $N=1$ Supersymmetric Yang-Mills in Four Dimensions," hep-th/0103011.

[69] J. A. Harvey and A. Strominger, "Octonionic superstring solitons," Phys. Rev. Lett. 66 (1991) 549.

[70] I. R. Klebanov and M. J. Strassler, "Supergravity and a confining gauge theory: Duality cascades and chiSB-resolution of naked singularities," JHEP 0008, 052 (2000).

[71] J. Polchinski, "N = 2 gauge-gravity duals," Int. J. Mod. Phys. A 16, 707 (2001).

[72] D. Joyce, "A New Construction of Compact 8-Manifolds with Holonomy Spin(7)," math.DG/9910002. 